\title{
Regioselective Synthesis of Multifunctional Allylic Amines; Access to Ambiphilic Aziridine Scaffolds
}

Dean D. Roberts and Mark G. McLaughlin*

Department of Natural Sciences, Manchester Metropolitan University, Manchester, M1 5GD

m.mclaughlin@mmu.ac.uk 


\section{Contents}

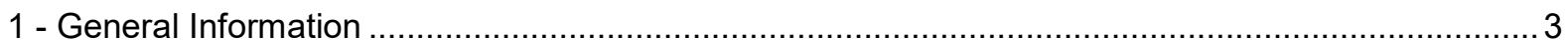

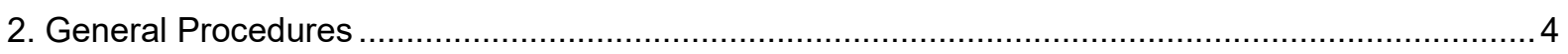

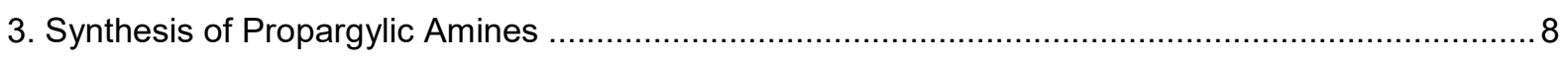

4. Synthesis of Propargylic Amides, Carbamates and Sulfonamides .............................................. 13

5. Platinum Catalysed Hydrosilylation of Propargylic Amines ..................................................... 20

6. Platinum Catalysed Hydrosilylation of Propargylic Amides, Carbamates and Sulfonamides...........26

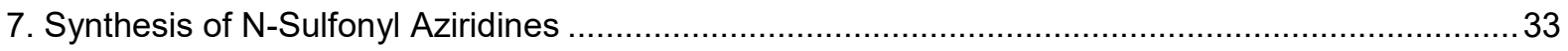

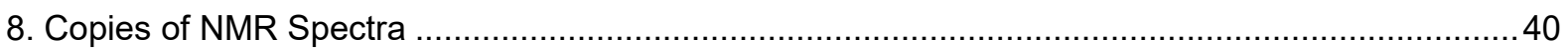




\section{1 - General Information}

\section{Solvents and reagents}

All solvents were purchased from commercial sources and used without purification (HPLC or analytical grade). Anhydrous solvent was obtained from a Pure Solv ${ }^{\mathrm{TM}}$ Solvent Purification System. Standard vacuum line techniques were used and glassware was oven dried prior to use. Organic solvents were dried during workup using anhydrous $\mathrm{Na}_{2} \mathrm{SO}_{4}$. All reactions were performed using DrySyn heating mantles and pressure regulated vials

\section{Purification and chromatography}

Thin Layer Chromatography (TLC) was carried out using aluminium plates coated with 60 F254 silica gel. Plates were visualised using UV light $(254$ or $365 \mathrm{~nm})$ or staining with $1 \%$ aq. $\mathrm{KMnO}_{4}$. Normal phase silica gel chromatography was carried out using either a Biotage Isolera One flash column chromatography system (LPLC) or traditional flash column chromatography using Geduran® Silica gel 60, 40-63 microns RE.

\section{Characterisation}

Infrared spectroscopy was carried out with a Nicolet $₫ 380$ FT/IR - Fourier Transform Infrared Spectrometer. Only the most significant frequencies have been considered during the characterization and selected absorption maxima (vmax) recorded in waves (cm-1 ). NMR spectra were recorded using a JEOL ${ }^{\circledR}$ ECS $-400 \mathrm{MHz}$ spectrometer using the deuterated solvent stated. Chemical shifts $(\delta)$ quoted in parts per million $(\mathrm{ppm})$ and referenced to the residual solvent peak. Multiplicities are denoted as s- singlet, d- doublet, t- triplet, q- quartet and quin- quintet and derivatives thereof (br denotes a broad resonance peak). Coupling constants recorded as $\mathrm{Hz}$ and round to the nearest $0.1 \mathrm{~Hz}$. High Resolution Mass Spectrometry (HOURMS) was recorded using an Agilent Technologies $₫ 6540$ Ultra-HighDefinition (UHD) AccurateMass equipped with a time of flight (Q-TOF) analyzer and the samples were ionized by ESI techniques and introduced thorough a high pressure liquid chromatography (HPLC) model Agilent Technologies ${ }^{\circledR} 1260$ Infinity Quaternary LC system. Compound names were generated using ACD/iLab systematic naming 


\section{General Procedures}

\section{General Procedure A-Synthesis of propargyl amines by substitution of propargyl bromide $^{1}$}

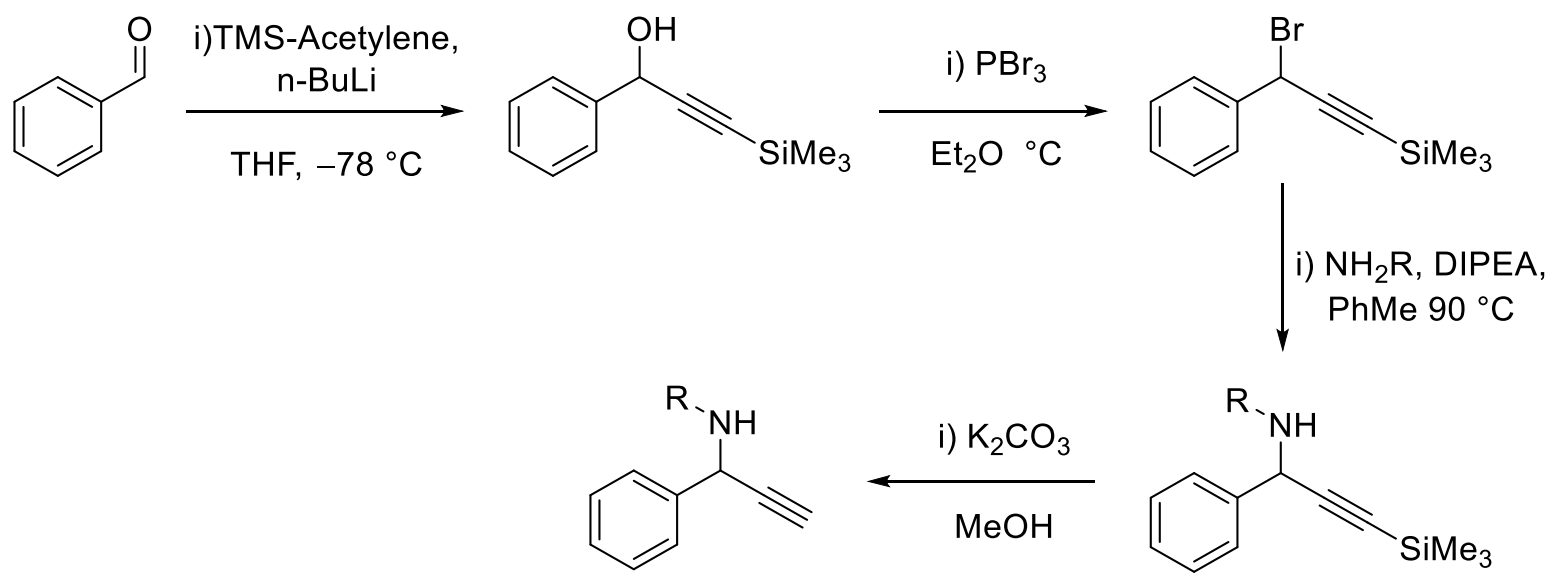

To a solution of trimethylsilylacetylene in THF $(0.5 \mathrm{M})$ under an argon atmosphere, n-BuLi (15 $\mathrm{mL}, 2.5 \mathrm{M}$ in hexane) was added dropwise at $-78^{\circ} \mathrm{C}$. The mixture was stirred for 30 minutes before the requisite aldehyde was added and the mixture was slowly warmed to room temperature before stirring for 2 hours. After this time the reaction was quenched with $\mathrm{NH}_{4} \mathrm{Cl}$, and extracted into DCM $(3 \times 10 \mathrm{~mL})$ and dried over $\mathrm{MgSO}_{4}$ before the solvent was removed under reduced pressure to yield the corresponding propargyl alcohol.

To a solution of propargyl alcohol in $\mathrm{Et}_{2} \mathrm{O}(1 \mathrm{M}), \mathrm{PBr}_{3}$ (3 equiv.) was dropwise added under an argon atmosphere at $0{ }^{\circ} \mathrm{C}$, and the mixture was stirred at the same temperature for $0.5 \mathrm{~h}$. The reaction mixture was poured into an ice bath and neutralized with saturated $\mathrm{NaHCO}_{3}$ aq. The two layers were separated, and the aqueous phase was extracted with EtOAc $(3 \times 10 \mathrm{~mL})$ before the combined organic layers were dried over $\mathrm{MgSO}_{4}$. The solvent was removed under reduced pressure to afford the corresponding propargyl bromide.

To a solution of amine (1.2 equiv.) in toluene $(0.5 \mathrm{M})$ under an argon atmosphere was added the corresponding propargyl bromide and diisopropylethylamine (1.2 equiv.). The reaction mixture was stirred for $24 \mathrm{~h}$ at $90{ }^{\circ} \mathrm{C}$. The reaction was then diluted with EtOAc (10 mL) and a saturated aqueous solution $(10 \mathrm{~mL})$ of $\mathrm{NaHCO}_{3}$ was added, before being extracted into EtOAc ( $3 \times 10 \mathrm{~mL}$.) The organic layer was separated and dried over $\mathrm{Na}_{2} \mathrm{SO}_{4}$ before the solvent was removed under reduced pressure.

To a solution of propargyl amine in methanol $(0.5 \mathrm{M})$ was added $\mathrm{K}_{2} \mathrm{CO}_{3}$ (3 equiv.). This solution was stirred at room temperature for 2 hours. After this time, the reaction was quenched with $\mathrm{H}_{2} \mathrm{O}(10 \mathrm{~mL})$ and EtOAc $(10 \mathrm{~mL})$, before being extracted into EtOAc $(3 \times 10 \mathrm{~mL}$.) The extract was dried with $\mathrm{Na}_{2} \mathrm{SO}_{4}$ before removal of solvent in vacuo. The residue was then purified via column chromatography to afford the corresponding propargyl amine. Yields are reported over 4 steps. 
General Procedure B-Synthesis of propargylic amines by $\mathrm{Cu}(\mathrm{l})$ catalysed $\underline{\text { amination }}^{2}$<smiles>[R]C([R])(O)C#CC[R16](C)=O</smiles>

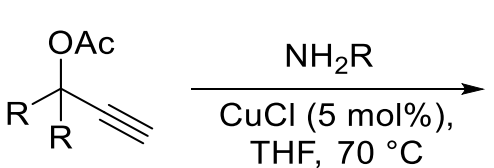<smiles>[R]NC([R])([R])C#C</smiles>

To a solution of propargylic alcohol in DCM (0.5 M) was added acetic anhydride (1.5 eq), triethylamine (2 equiv.) and DMAP (0.2 equiv.). This solution was stirred until TLC analysis showed complete consumption of the starting material. After this time, the solution was quenched with water $(10 \mathrm{~mL})$, and extracted into DCM $(3 \times 10 \mathrm{~mL}$.) The extract was dried with $\mathrm{Na}_{2} \mathrm{SO}_{4}$ before removal of solvent in vacuo.

To a solution of copper ( $\mathrm{I})$ chloride $(5 \mathrm{~mol} \%)$ in THF ( $0.5 \mathrm{M})$ was added propargyl acetate and the requisite amine (1.5 equiv.). This solution was heated to reflux under argon for 2 hours, after which time the solution was concentrated and purified via column chromatography (EtOAc:Hex) to afford the pure product. Yields are reported over 2 steps.

\section{General Procedure C-Synthesis of propargyl amines by organolithium addition $\underline{\text { to }} \mathrm{C}=\mathrm{N}$ bonds $^{3}$}

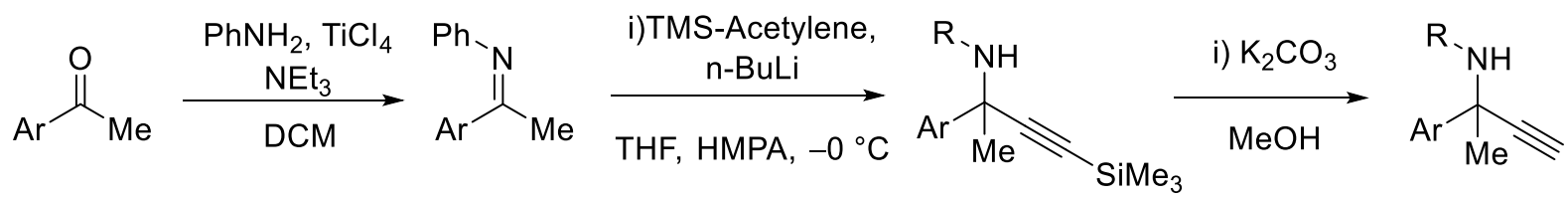

To a solution of the requisite ketone dissolved in toluene $(1 \mathrm{M})$ with $3 \AA$ molecular sieves was added aniline (1.1 eq) under an argon atmosphere. This solution was then heated at reflux for 24 hours, after which time the solution was filtered before volatiles were removed in vacuo. The resultant residue was then triturated with ethanol, which resulted in precipitation of the corresponding imine as a pale yellow powder.

To a solution of trimethylsilylacetylene (1.3 equiv.) in THF (1 M) was added $n-B u L i$ (1.2 equiv.) at $0{ }^{\circ} \mathrm{C}$. This solution was then stirred at the same temperature for 30 minutes before hexamethylphosphoramide ( $3 \mathrm{eq})$ and ketimine were added to the reaction mixture. The resultant black solution was slowly warmed to room temperature before being stirred for a further $2 \mathrm{~h}$. After this time, the reaction was quenched with $\mathrm{NH}_{4} \mathrm{Cl}$ before being extracted into a 4:1 mixture of Hexane:Ethyl Acetate $\left(3 \times 10 \mathrm{~mL}\right.$.) The extract was dried over $\mathrm{Na}_{2} \mathrm{SO}_{4}$ before removal of solvent in vacuo.

To a solution of propargyl amine in methanol $(0.5 \mathrm{M})$ was added $\mathrm{K}_{2} \mathrm{CO}_{3}$ (3 equiv.). This solution was stirred at room temperature for 2 hours. After this time, the reaction was quenched with $\mathrm{H}_{2} \mathrm{O}(10 \mathrm{~mL})$ and EtOAc $(10 \mathrm{~mL})$, before being extracted into EtOAc $(3 \times 10 \mathrm{~mL}$.) The extract was dried with $\mathrm{Na}_{2} \mathrm{SO}_{4}$ before removal of solvent in vacuo. The residue was then purified via column chromatography to afford the pure product. Yields are reported over 3 steps. 


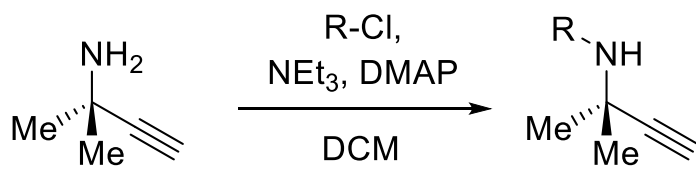

To a solution of 2-methyl-3-butyn-2-amine, TEA and DMAP in DCM (1M) was added the corresponding acyl chloride, sulfonyl chloride or anhydride (1 equiv.) at $0{ }^{\circ} \mathrm{C}$. The solution was then allowed to warm to room temperature, before stirring overnight. The reaction was then quenched with water $(5 \mathrm{~mL})$ before being extracted into EtOAc $(3 \times 5 \mathrm{~mL}$.) The combined organic layers were washed with brine, dried with $\mathrm{Na}_{2} \mathrm{SO}_{4}$, filtered and concentrated. Flash column chromatography afforded the pure product.

\section{General Procedure E- Hydrosilylation of propargyl amines, amides and sulfonamides}<smiles>[R]S(=O)(=O)NC(C)(C)C#C</smiles>

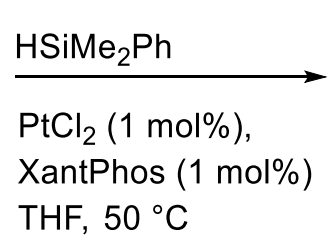<smiles>[R]S(=O)(=O)NC(C)(C)/C=C/Sc1ccccc1</smiles>

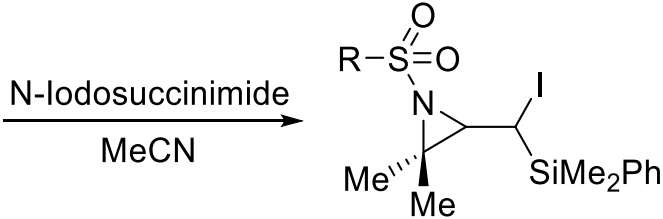

To an oven dried $22 \mathrm{~mL}$ vial and magnetic stirrer was added $\mathrm{PtCl}_{2}$ (1 mol\%) and 4,5Bis(diphenylphosphino)-9,9-dimethylxanthene (1 mol\%) (XantPhos). The flask was then flushed quickly with argon and dry THF was added (0.3M). The mixture was then stirred at 50 ${ }^{\circ} \mathrm{C}$ for 30 minutes until a yellow homogenous mixture was obtained. The corresponding propargyl amine (1 equiv.) was added followed by the silane (1.5 equiv.) via syringe and the solution was stirred at $50{ }^{\circ} \mathrm{C}$ overnight. The solvent was evaporated and the crude mixture was applied to the top of a column and chromatographed to afford the requisite vinyl silane.

\section{General Procedure F- lodoaziridination of vinyl silanes}<smiles>[R]S(=O)(=O)NC(C)(C)/C=C/[AsH2+]c1ccccc1</smiles>

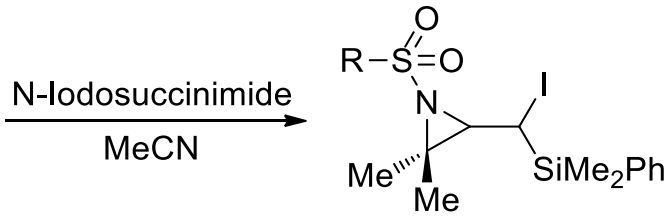

To an oven dried vial was added vinyl silane and $\mathrm{N}$-lodosuccinimide ( 3 equiv.) The vial was then flushed with argon and dry acetonitrile $(0.2 \mathrm{M})$ was added. The mixture was then stirred overnight at room temperature. The reaction was then quenched with saturated $\mathrm{Na}_{2} \mathrm{~S}_{2} \mathrm{O}_{3}$. The mixture was then extracted into DCM $(3 \times 5 \mathrm{~mL})$ and the solvent removed under reduced pressure. The residue was then purified by column chromatography to afford the corresponding N-Sulfonyl aziridine. 


\section{Telescoped Hydrosilylation-Cyclisation Procedure}

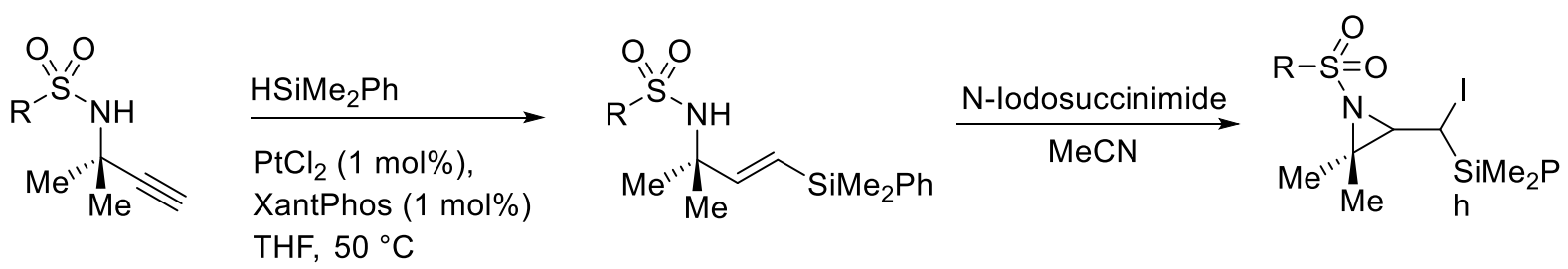

To an oven dried $22 \mathrm{~mL}$ vial and magnetic stirrer was added $\mathrm{PtCl}_{2}$ (1 mol\%) and 4,5Bis(diphenylphosphino)-9,9-dimethylxanthene (1 mol\%) (XantPhos). The flask was then flushed quickly with argon and dry THF was added (0.3M). The mixture was then stirred at 50 ${ }^{\circ} \mathrm{C}$ for 30 minutes until a yellow homogenous mixture was obtained. $3 e$ ( 1 equiv.) was added followed by the silane ( 1.5 equiv.) via syringe and the solution was stirred at $50{ }^{\circ} \mathrm{C}$ overnight. The solvent was evaporated and the crude mixture was applied to the top of a column and chromatographed to afford the requisite vinyl silane.

To an oven dried vial was added vinyl silane and $\mathrm{N}$-lodosuccinimide ( 3 equiv.) The vial was then flushed with argon and dry acetonitrile $(0.2 \mathrm{M})$ was added. The mixture was then stirred overnight at room temperature. The reaction was then quenched with saturated $\mathrm{Na}_{2} \mathrm{~S}_{2} \mathrm{O}_{3}$. The mixture was then extracted into DCM $(3 \times 5 \mathrm{~mL})$ and the solvent removed under reduced pressure. The residue was then purified by column chromatography to afford the corresponding N-Sulfonyl aziridine.

\section{Scaled-Up Hydrosilylation Procedure (1 mmol)}

To an oven dried $22 \mathrm{~mL}$ vial and magnetic stirrer was added $\mathrm{PtCl}_{2}(2.6 \mathrm{mg}, 0.01$ equiv.) and 4,5-Bis(diphenylphosphino)-9,9-dimethylxanthene ( $5.6 \mathrm{mg}, 0.01$ equiv.) (XantPhos). The flask was then flushed quickly with argon and dry THF was added (0.3M). The mixture was then stirred at $50{ }^{\circ} \mathrm{C}$ for 30 minutes until a yellow homogenous mixture was obtained. The corresponding propargylic sulfonamide $(247 \mathrm{mg}, 1 \mathrm{mmol})$ was added followed by the silane (200 mg, $1.5 \mathrm{mmol}$ ) via syringe and the solution was stirred at $50{ }^{\circ} \mathrm{C}$ overnight. The solvent was evaporated and the crude mixture was applied to the top of a column and chromatographed to afford the requisite vinyl silane as a white solid $(274 \mathrm{mg}, 74 \%$.) 


\section{Synthesis of Propargylic Amines}

\section{N-benzyl-1-phenylprop-2-yn-1-amine (1a)}<smiles>C#CC(NCc1ccccc1)c1ccccc1</smiles>

The title compound was prepared according to general procedure $\mathrm{A}$, from benzaldehyde $(1 \mathrm{~g}$, $9.4 \mathrm{mmol}$ ) and benzylamine. Following conversion to the product and column chromatography (1:9 EtOAc:hexane) afforded 1a as a yellow oil. (453 mg, 22\%)

${ }^{1} \mathrm{H}$ NMR $\left(400 \mathrm{MHz}, \mathrm{CDCl}_{3}\right) \delta 7.55(\mathrm{~d}, J=7.6 \mathrm{~Hz}, 2 \mathrm{H}), 7.41-7.22(\mathrm{~m}, 8 \mathrm{H}), 4.58(\mathrm{~d}, J=2.0 \mathrm{~Hz}$, $1 \mathrm{H}), 4.12-3.75(\mathrm{~m}, 2 \mathrm{H}), 2.54(\mathrm{~d}, J=2.3 \mathrm{~Hz}, 1 \mathrm{H}), 1.71(\mathrm{~s}, 1 \mathrm{H})$

${ }^{13} \mathrm{C}$ NMR $\left(101 \mathrm{MHz}, \mathrm{CDCl}_{3}\right) \delta 139.7,139.57,128.5,128.4,127.8,127.5,127.1,126.5,83.6$, 76.7, 73.6, 52.8, 50.9.

*Data in accordance with literature. ${ }^{1}$

\section{$\underline{\mathrm{N}-[2-p h e n y l b u t-3-y n-2-y l] a n i l i n e ~(1 b)}$}<smiles>C#CC(Nc1ccccc1)c1ccccc1</smiles>

The title compound was prepared according to general procedure A, from benzaldehyde $(1 \mathrm{~g}$, $9.4 \mathrm{mmol})$ and aniline. Following conversion to the product and column chromatography (1:9 EtOAc:hexane) afforded $\mathbf{1 b}$ as a colourless oil. (534 mg, 27\%)

${ }^{1} \mathrm{H} \mathrm{NMR}\left(400 \mathrm{MHz}, \mathrm{CDCl}_{3}\right) \delta 7.60(\mathrm{~d}, J=7.2 \mathrm{~Hz}, 2 \mathrm{H}), 7.36(\mathrm{dt}, J=22.9,7.2 \mathrm{~Hz}, 3 \mathrm{H}), 7.21$ (dd, $J=8.3,7.5 \mathrm{~Hz}, 2 \mathrm{H}), 6.79(\mathrm{t}, J=7.4 \mathrm{~Hz}, 1 \mathrm{H}), 6.73(\mathrm{~d}, J=7.7 \mathrm{~Hz}, 2 \mathrm{H}), 5.29(\mathrm{~s}, 1 \mathrm{H}), 4.05$ (s, 1H), $2.47(\mathrm{~d}, J=2.3 \mathrm{~Hz}, 1 \mathrm{H})$.

${ }^{13} \mathrm{C}$ NMR $\left(101 \mathrm{MHz}, \mathrm{CDCl}_{3}\right) \delta 145.0,143.9,128.7,128.6,127.4,125.6,118.5,115.8,86.1$, $72.4,55.3,35.9$.

${ }^{*}$ Data in accordance with literature. ${ }^{1}$ 


\section{N-benzyl-2-(2-fluorophenyl)but-3-yn-2-amine (1c)}<smiles>C#CC(C)(NCc1ccccc1)c1ccccc1F</smiles>

The title compound was prepared according to general procedure A, from 2fluoroacetophenone $(500 \mathrm{mg}, 3.62 \mathrm{mmol})$, and benzylamine. Following conversion to the product and column chromatography (1:9 EtOAc:hexane) afforded 1c as a colourless oil. (153 $\mathrm{mg}, 17 \%)$

Rf (9:1 Hex:EtOAc) 0.22

IR vmax $\left(\mathrm{cm}^{-1}\right)$ 3543, 3097, 3065, 3001, 2974

HRMS (ESI) m/z: [M + H]+ Calcd for $\mathrm{C}_{17} \mathrm{H}_{16} \mathrm{NF} 254.1345$; Found 254.1341

${ }^{1} \mathrm{H}$ NMR $\left(400 \mathrm{MHz}, \mathrm{CDCl}_{3}\right) \delta 7.89-7.81(\mathrm{~m}, 1 \mathrm{H}), 7.34-7.21(\mathrm{~m}, 6 \mathrm{H}), 7.14(\mathrm{t}, J=7.6 \mathrm{~Hz}$, $1 \mathrm{H}), 7.05(\mathrm{dd}, J=12.4,8.1 \mathrm{~Hz}, 1 \mathrm{H}), 3.90(\mathrm{~d}, J=12.2 \mathrm{~Hz}, 1 \mathrm{H}), 3.46(\mathrm{~d}, J=12.3 \mathrm{~Hz}, 1 \mathrm{H})$,

$2.66(\mathrm{~s}, 1 \mathrm{H}), 1.84(\mathrm{~s}, 3 \mathrm{H})$.

${ }^{13} \mathrm{C}$ NMR (101 MHz, ) $\delta 160.9$ (d, 248.3 Hz), 153.0, 140.9, 139.0, 134.0 129.0, 128.9, 128.8, $128.51,128.4,127.8,127.0,124.7,124.0,116.5,116.3,61.59,48.0,25.4,-2.30$.

\section{N-benzyl-3,4,4-trimethylpent-1-yn-3-amine (1d)}

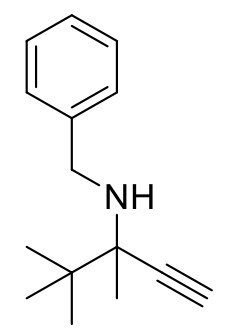

The title compound was prepared according to general procedure A, from pinacolone (500 $\mathrm{mg}, 5 \mathrm{mmol}$ ) and benzylamine. Following conversion to the product and column chromatography (1:9 EtOAc:hexane) afforded 1d as a yellow oil. (303 mg, 28 \%)

Rf (9:1 Hex:EtOAc) 0.26

IR umax $\left(\mathrm{cm}^{-1}\right)$ 3541, 3054, 3025, 2999, 2992

HRMS (ESI) m/z: [M + H]+ Calcd for $\mathrm{C}_{15} \mathrm{H}_{21} \mathrm{~N} 216.1758$; Found 216.1755

${ }^{1} \mathrm{H} \mathrm{NMR}\left(400 \mathrm{MHz}, \mathrm{CDCl}_{3}\right) \delta 7.39(\mathrm{~d}, J=7.4 \mathrm{~Hz}, 2 \mathrm{H}), 7.32(\mathrm{t}, J=7.5 \mathrm{~Hz}, 2 \mathrm{H}), 7.23(\mathrm{~d}, J=7.2$ $\mathrm{Hz}, 1 \mathrm{H}$ ), 3.88 (dd, $J=66.7,12.4 \mathrm{~Hz}, 2 \mathrm{H}), 2.35$ (s, 1H), 1.31 (s, 3H), $1.06(\mathrm{~s}, 9 \mathrm{H})$.

${ }^{13} \mathrm{C}$ NMR $\left(101 \mathrm{MHz}, \mathrm{CDCl}_{3}\right) \delta 141.7,128.5,128.4,126.9,87.9,71.7,60.2,48.9,37.6,25.5$, 21.3. 


\section{N-benzyl-1-ethynylcyclohexan-1-amine (1e)}<smiles>C#CC1(NCc2ccccc2)CCCCC1</smiles>

The title compound was prepared according to general procedure $\mathrm{B}$, from 1-ethynyl-1cyclohexanol ( $250 \mathrm{mg}, 2 \mathrm{mmol}$ ) and benzylamine. Following conversion to the product and column chromatography (1:9 EtOAc:hexane) afforded 1e as a colourless oil. (254 mg, 59\%)

${ }^{1} \mathrm{H}$ NMR (400 MHz, $\left.\mathrm{CDCl}_{3}\right) \delta 7.40(\mathrm{~d}, J=7.2 \mathrm{~Hz}, 2 \mathrm{H}), 7.35$ (d, J=7.4 Hz, 2H), $7.30-7.25$ (m, 1H), $3.91(\mathrm{~s}, 2 \mathrm{H}), 2.43(\mathrm{~s}, 1 \mathrm{H}), 1.91(\mathrm{~d}, J=12.5 \mathrm{~Hz}, 2 \mathrm{H}), 1.74-1.61(\mathrm{~m}, 5 \mathrm{H}), 1.48(\mathrm{td}, J=$ 12.0, 3.7 Hz, 2H), 1.27 (dd, $J=15.3,8.1 \mathrm{~Hz}, 2 \mathrm{H})$.

${ }^{13} \mathrm{C}$ NMR $\left(101 \mathrm{MHz}, \mathrm{CDCl}_{3}\right) \delta 141.0,128.6,128.5,127.0,88.1,72.1,54.8,48.0,38.0,25.9$, 22.8 .

${ }^{*}$ Data in accordance with literature. ${ }^{2}$

\section{$\underline{\mathrm{N} \text {-benzyl-3,5-dimethylhex-1-yn-3-amine (1f) }}$}<smiles>C#CC(C)(CC(C)C)NCc1ccccc1</smiles>

The title compound was prepared according to general procedure B from 3,5-dimethylhex-1yn-3-ol (250 mg, $2 \mathrm{mmol}$ ) and benzylamine. Following conversion to the product and column chromatography (1:9 EtOAc:hexane) afforded $1 \mathrm{f}$ as a colourless oil. (180 mg, 43\%)

${ }^{1} \mathrm{H}$ NMR $\left(400 \mathrm{MHz}, \mathrm{CDCl}_{3}\right) \delta 7.39-7.27(\mathrm{~m}, 3 \mathrm{H}), 7.23(\mathrm{dd}, J=5.9,3.6 \mathrm{~Hz}, 2 \mathrm{H}), 3.86(\mathrm{dd}, J=$ 27.8, 11.9 Hz, 2H), $2.37(\mathrm{~s}, 1 \mathrm{H}), 1.90(\mathrm{td}, J=12.8,6.4 \mathrm{~Hz}, 1 \mathrm{H}), 1.63-1.50(\mathrm{~m}, 2 \mathrm{H}), 1.39(\mathrm{~s}$, $3 \mathrm{H}), 1.00(\mathrm{~d}, J=6.7 \mathrm{~Hz}, 6 \mathrm{H})$.

${ }^{13} \mathrm{C}$ NMR $\left(101 \mathrm{MHz}, \mathrm{CDCl}_{3}\right) \delta 140.7,128.5,128.5,127.1,83.6,68.1,54.1,50.4,48.6,27.3$, 25.7, 24.7, 24.7, 24.6.

${ }^{*}$ Data in accordance with literature. ${ }^{2}$ 


\section{$\underline{N-(1-p h e n y l p r o p-2-y n-1-y l) a n i l i n e ~(1 g) ~}$}<smiles>C#CC(C)(Nc1ccccc1)c1ccccc1</smiles>

The title compound was prepared according to general procedure $C$ from acetophenone (500 $\mathrm{mg}, 4.2 \mathrm{mmol}$ ) and aniline. Following conversion to the product and column chromatography (1:9 EtOAc:hexane) afforded $\mathbf{1 g}$ as a white solid. (481 $\mathrm{mg}, 53 \%)$

${ }^{1} \mathrm{H}$ NMR $\left(400 \mathrm{MHz}, \mathrm{CDCl}_{3}\right) \delta 7.60(\mathrm{~d}, J=7.2 \mathrm{~Hz}, 2 \mathrm{H}), 7.36(\mathrm{dt}, J=22.9,7.2 \mathrm{~Hz}, 3 \mathrm{H}), 7.21$ (dd, $J=8.3,7.5 \mathrm{~Hz}, 2 \mathrm{H}), 6.79(\mathrm{t}, J=7.4 \mathrm{~Hz}, 1 \mathrm{H}), 6.73(\mathrm{~d}, J=7.7 \mathrm{~Hz}, 2 \mathrm{H}), 5.29(\mathrm{~s}, 1 \mathrm{H}), 4.05(\mathrm{~s}$, $1 \mathrm{H}), 2.47(\mathrm{~d}, J=2.3 \mathrm{~Hz}, 1 \mathrm{H})$.

${ }^{13} \mathrm{C}$ NMR $\left(101 \mathrm{MHz}, \mathrm{CDCl}_{3}\right) \delta 146.4,139.1,129.3,128.9,128.3,127.3,118.9,114.1,83.1$, 73.2, 49.9., 35.0 .

Data in accordance with literature. ${ }^{4}$

\section{$\underline{\mathrm{N}-[2-(4-m e t h y l p h e n y l) b u t-3-y n-2-y l] a n i l i n e ~(1 \mathrm{~h})}$}<smiles>C#CC(C)(Nc1ccccc1)c1ccc(C)cc1</smiles>

The title compound was prepared according to general procedure C from 4methylacetophenone (500 mg, $3.7 \mathrm{mmol}$ ) and aniline. Following conversion to the product and column chromatography (1:9 EtOAc:hexane) afforded $1 \mathrm{~h}$ as a white solid. ( $579 \mathrm{mg}, 66 \%)$

${ }^{1} \mathrm{H}$ NMR $\left(400 \mathrm{MHz}, \mathrm{CDCl}_{3}\right) \delta 7.56(\mathrm{~d}, J=8.2 \mathrm{~Hz}, 2 \mathrm{H}), 7.13(\mathrm{~d}, J=8.3 \mathrm{~Hz}, 2 \mathrm{H}), 7.05(\mathrm{dd}, J=$ 8.5, 7.4 Hz, 2H), $6.68(\mathrm{~s}, 1 \mathrm{H}), 6.53(\mathrm{~d}, J=7.8 \mathrm{~Hz}, 2 \mathrm{H}), 4.22(\mathrm{~s}, 1 \mathrm{H}), 2.46(\mathrm{~s}, 1 \mathrm{H}), 2.33(\mathrm{~s}, 3 \mathrm{H})$, $1.78(\mathrm{~s}, 3 \mathrm{H})$.

${ }^{13} \mathrm{C}$ NMR $\left(101 \mathrm{MHz}, \mathrm{CDCl}_{3}\right) \delta 145.2,141.1,137.1,129.4,128.7,125.5,118.5,115.9,86.3$, $72.3,55.2,36.0,21.2$.

Data in accordance with literature. ${ }^{4}$ 


\section{$\underline{N-(3,5-d i m e t h y l h e x-1-y n-3-y l)-4-(t r i f l u o r o m e t h y l) a n i l i n e ~(1 i) ~}$}<smiles>C#CC(C)(CC(C)C)Nc1ccc(C(F)(F)F)cc1</smiles>

The title compound was prepared according to general procedure B from 3,5-dimethyl-hex-1yn-3-ol and 4-trifluoromethylaniline (250 mg, 2 mmol.) Following conversion to the product and column chromatography (1:9 EtOAc:hexane) afforded $1 \mathrm{i}$ as an orange oil. (398 $\mathrm{mg}, 74 \%$ )

IR umax $\left(\mathrm{cm}^{-1}\right)$ 3512, 3004, 2994, 2983, 2970

HRMS (ESI) m/z: [M + H] + Calcd for $\mathrm{C}_{15} \mathrm{H}_{18} \mathrm{~F}_{3} \mathrm{~N} 270.1470$; Found 270.1475

${ }^{1} \mathrm{H}$ NMR $\left(400 \mathrm{MHz}, \mathrm{CDCl}_{3}\right) \delta 7.41(\mathrm{~d}, J=8.6 \mathrm{~Hz}, 2 \mathrm{H}), 6.91(\mathrm{~d}, J=8.5 \mathrm{~Hz}, 2 \mathrm{H}), 2.44(\mathrm{~s}, 1 \mathrm{H})$, $1.98(\mathrm{dt}, J=12.7,6.3 \mathrm{~Hz}, 1 \mathrm{H}), 1.76(\mathrm{ddd}, J=42.5,14.0,5.9 \mathrm{~Hz}, 2 \mathrm{H}), 1.60(\mathrm{~d}, J=10.4 \mathrm{~Hz}$, $3 \mathrm{H}), 1.03$ (dd, $J=25.1,6.7 \mathrm{~Hz}, 6 \mathrm{H})$.

${ }^{13} \mathrm{C}$ NMR (101 MHz, $\left.\mathrm{CDCl}_{3}\right) \delta 148.15,126.3$, (q, J=7.0 Hz), 123.7, 119.4 (q, J = $\left.32.6 \mathrm{~Hz}\right)$, 114.6, 86.6, 72.5, 51.2, 50.4, 28.6, 25.0, 24.5.

${ }^{19} \mathrm{~F}$ NMR $\left(376 \mathrm{MHz}, \mathrm{CDCl}_{3}\right) \delta-61.04$.

\section{$\underline{N-[(3-c h l o r o p h e n y l) m e t h y l]-1-p h e n y l p r o p-2-y n-1-a m i n e ~(1 i) ~}$}<smiles>C#CC(NCc1cccc(Cl)c1)c1ccccc1</smiles>

The title compound was prepared according to general procedure A from benzaldehyde $(1 \mathrm{~g}$, $9.4 \mathrm{mmol}$ ) and 3-chlorobenzylamine. Following conversion to the product and column chromatography (1:9 EtOAc:hexane) afforded $1 \mathbf{j}$ as a yellow oil. (468 mg, 22\%)

${ }^{1} \mathrm{H}$ NMR $\left(400 \mathrm{MHz}, \mathrm{CDCl}_{3}\right) \delta 7.60-7.53(\mathrm{~m}, 2 \mathrm{H}), 7.41-7.34(\mathrm{~m}, 3 \mathrm{H}), 7.33-7.30(\mathrm{~m}, 1 \mathrm{H})$, $7.28-7.22(\mathrm{~m}, 3 \mathrm{H}), 4.58(\mathrm{~d}, J=2.2 \mathrm{~Hz}, 1 \mathrm{H}), 3.96-3.86(\mathrm{~m}, 2 \mathrm{H}), 2.56(\mathrm{~d}, J=2.3 \mathrm{~Hz}, 1 \mathrm{H})$.

${ }^{13} \mathrm{C}$ NMR $\left(101 \mathrm{MHz}, \mathrm{CDCl}_{3}\right) \delta 141.9,139.6,134.4,129.8,128.7,128.6,128.1,127.6,127.4$, $126.6,83.5,73.9,53.0,50.5$.

Data in accordance with literature. ${ }^{1}$ 


\section{2-chloro-4-methyl-N-(1-phenylprop-2-yn-1-yl)aniline (1k)}<smiles>C#CC(Nc1ccc(C)cc1Cl)c1ccccc1</smiles>

The title compound was prepared according to general procedure A from benzaldehyde $(1 \mathrm{~g}$, $9.4 \mathrm{mmol}$ ) and 2-chloro-4-methylaniline. Following conversion to the product and column chromatography (1:9 EtOAc:hexane) afforded $\mathbf{1 k}$ as an orange oil. (487 mg, 21\%)

1H NMR (400 MHz,CDCl $\left.{ }_{3}\right) \delta 7.65-7.58(\mathrm{~m}, 2 \mathrm{H}), 7.46-7.31(\mathrm{~m}, 3 \mathrm{H}), 7.12(\mathrm{~d}, J=1.6 \mathrm{~Hz}$, $1 \mathrm{H}), 6.96(\mathrm{dd}, J=8.2,1.5 \mathrm{~Hz}, 1 \mathrm{H}), 6.76(\mathrm{~d}, J=8.3 \mathrm{~Hz}, 1 \mathrm{H}), 5.31(\mathrm{dd}, J=6.9,1.8 \mathrm{~Hz}, 1 \mathrm{H})$, $4.60(\mathrm{~d}, J=6.8 \mathrm{~Hz}, 1 \mathrm{H}), 2.49(\mathrm{~d}, J=2.3 \mathrm{~Hz}, 1 \mathrm{H}), 2.24(\mathrm{~s}, 3 \mathrm{H})$.

${ }^{13} \mathrm{C}$ NMR $\left(101 \mathrm{MHz}, \mathrm{CDCl}_{3}\right) \delta 147.5,141.3,138.50,129.59,128.8,128.1,127.8,127.5,127.2$, $127.1,84.3,73.7,62.4,20.2$.

Data in accordance with literature. ${ }^{1}$

\section{$\underline{N-[(4-m e t h o x y p h e n y l) m e t h y l]-2-m e t h y l b u t-3-y n-2-a m i n e ~(1 I) ~}$}<smiles>C#CC(C)(C)NCc1ccc(OC)cc1</smiles>

To a solution of 4-methoxybenzaldehyde $(160 \mathrm{mg}, 0.6 \mathrm{mmol})$ in methanol $(2 \mathrm{~mL})$ was added 1,1 dimethyl propargyl amine $(50 \mathrm{mg}, 0.6 \mathrm{mmol})$. The solution was stirred overnight at room temperature. To this solution was added $\mathrm{NaBH}_{4}$ and the reaction was stirred for 1 hour at room temperature. After this time, the reaction mixture was quenched with water $(5 \mathrm{~mL})$ and extracted into DCM $(3 \times 5 \mathrm{~mL})$, before volatiles were removed under vacuum. The resultant mixture was then purified by flash column chromatography (EtOAc:Hex 9:1) to afford the 1I as a white solid.

IR umax $\left(\mathrm{cm}^{-1}\right)$ 3519, 3089, 3045, 3000, 2979

HRMS (ESI) m/z: [M + H]+ Calcd for $\mathrm{C}_{13} \mathrm{H}_{18} \mathrm{NO} 204.1388$; Found 204.1395

${ }^{1} \mathrm{H} \mathrm{NMR}\left(400 \mathrm{MHz}, \mathrm{CDCl}_{3}\right) \delta 7.27(\mathrm{~d}, J=8.5 \mathrm{~Hz}, 2 \mathrm{H}), 6.85(\mathrm{~d}, J=8.5 \mathrm{~Hz}, 2 \mathrm{H}), 3.80(\mathrm{~s}, 2 \mathrm{H})$, $3.78(\mathrm{~s}, 3 \mathrm{H}), 2.35(\mathrm{~s}, 1 \mathrm{H}), 1.41(\mathrm{~s}, 6 \mathrm{H})$.

${ }^{13} \mathrm{C}$ NMR $\left(101 \mathrm{MHz}, \mathrm{CDCl}_{3}\right) \delta$ 158.7, 132.8, 129.7, 113.9, 89.2, 69.9, 55.4, 50.1, 48.5, 29.6. 


\section{Synthesis of Propargylic Amides, Carbamates and Sulfonamides}

\section{$\underline{\mathrm{N} \text {-(2-methylbut-3-yn-2-yl)benzenesulfonamide (3a) }}$}<smiles>C#CC(C)(C)NS(=O)(=O)c1ccccc1</smiles>

The title compound was prepared according to general procedure $\mathrm{D}$, from benzenesulfonyl chloride $(210 \mathrm{mg}, 1.2 \mathrm{mmol})$. Following conversion to the product and column chromatography (1:9 EtOAc:hexane) afforded $3 \mathrm{a}$ as a white solid (200 mg, 75\%)

${ }^{1} \mathrm{H}$ NMR $\left(400 \mathrm{MHz}, \mathrm{CDCl}_{3}\right) \delta 8.03-7.87(\mathrm{~m}, 3 \mathrm{H}), 7.24-7.10(\mathrm{~m}, 2 \mathrm{H}), 4.75(\mathrm{~s}, 1 \mathrm{H}), 2.08(\mathrm{~s}$, $1 \mathrm{H}), 1.55(\mathrm{~s}, 6 \mathrm{H})$.

${ }^{13} \mathrm{C}$ NMR $\left(101 \mathrm{MHz}, \mathrm{CDCl}_{3}\right) \delta 130.6,130.5,116.1,115.8,85.2,71.6,50.2,30.8$.

Data in accordance with literature. ${ }^{5}$

\section{4-methyl-N-(2-methylbut-3-yn-2-yl)benzene-1-sulfonamide (3b)}<smiles>C#CC(C)(C)NS(=O)(=O)c1ccc(C)cc1</smiles>

The title compound was prepared according to general procedure $D$, from tosyl chloride (228 $\mathrm{mg}, 1.2 \mathrm{mmol})$. Following conversion to the product and column chromatography (1:9 EtOAc:hexane) afforded $\mathbf{3 b}$ as a white solid (193 mg, 68\%)

${ }^{1} \mathrm{H}$ NMR $\left(400 \mathrm{MHz}, \mathrm{CDCl}_{3}\right) \delta 7.78(\mathrm{~d}, J=8.3 \mathrm{~Hz}, 2 \mathrm{H}), 7.27(\mathrm{~d}, J=8.3 \mathrm{~Hz}, 2 \mathrm{H}), 4.82-4.59(\mathrm{~m}$, $1 \mathrm{H}), 2.41(\mathrm{~s}, 3 \mathrm{H}), 2.09(\mathrm{~d}, J=1.1 \mathrm{~Hz}, 1 \mathrm{H}), 1.54(\mathrm{~s}, 6 \mathrm{H})$.

${ }^{13} \mathrm{C}$ NMR $\left(101 \mathrm{MHz}, \mathrm{CDCl}_{3}\right) \delta 143.1,139.3,129.5,127.8,85.2,70.9,50.2,30.5,21.8$

Data in accordance with literature. ${ }^{5}$ 


\section{4-methoxy-N-(2-methylbut-3-yn-2-yl)benzene-1-sulfonamide (3c)}

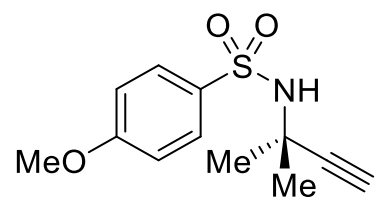

The title compound was prepared according to general procedure $\mathrm{D}$, from 4-methoxybenzene sulfonyl chloride $(247 \mathrm{mg}, 1.2 \mathrm{mmol})$. Following conversion to the product and column chromatography (1:9 EtOAc:hexane) afforded $3 \mathrm{c}$ as a white solid (282 $\mathrm{mg}, 93 \%)$

${ }^{1} \mathrm{H}$ NMR (400 MHz, $\left.\mathrm{CDCl}_{3}\right) \delta 7.93-7.72(\mathrm{~m}, 4 \mathrm{H}), 4.85(\mathrm{~s}, 1 \mathrm{H}), 3.85(\mathrm{~s}, 3 \mathrm{H}), 2.09(\mathrm{~s}, 1 \mathrm{H}), 1.53$ $(\mathrm{s}, 6 \mathrm{H})$.

${ }^{13} \mathrm{C}$ NMR $\left(101 \mathrm{MHz}, \mathrm{CDCl}_{3}\right) \delta 162.9,133.4,129.9,113.9,85.6,71.3,55.7,50.0,30.8$.

Data in accordance with literature. ${ }^{5}$

\section{2-chloro-N-(2-methylbut-3-yn-2-yl)benzene-1-sulfonamide (3d)}<smiles>C#CC(C)(C)NS(=O)(=O)c1ccccc1Cl</smiles>

The title compound was prepared according to general procedure $\mathrm{D}$, from 2-chlorobenzene sulfonyl chloride (253 $\mathrm{mg}, 1.2 \mathrm{mmol}$ ) Following conversion to the product and column chromatography (1:9 EtOAc:hexane) afforded $\mathbf{3 d}$ as a white solid (200 mg, 65\%)

${ }^{1} \mathrm{H} \mathrm{NMR}\left(400 \mathrm{MHz}, \mathrm{CDCl}_{3}\right)$ ठ 7.91 (dd, J = 5.4, 3.4 Hz, 1H), $7.57-7.45(\mathrm{~m}, 2 \mathrm{H}), 7.25(\mathrm{~s}, 1 \mathrm{H})$, $4.83(\mathrm{~s}, 1 \mathrm{H}), 2.05(\mathrm{~s}, 1 \mathrm{H}), 1.55(\mathrm{~s}, 6 \mathrm{H})$.

${ }^{13} \mathrm{C}$ NMR $\left(101 \mathrm{MHz}, \mathrm{CDCl}_{3}\right) \delta 141.6,137.6,135.3$ 132.6, 128.8, 127.7, 85.3, 71.4, 50.1, 30.8.

Data in accordance with literature. ${ }^{5}$ 


\section{3-chloro-N-(2-methylbut-3-yn-2-yl)benzene-1-sulfonamide (3e)}<smiles>C#CC(C)(C)NS(=O)(=O)c1cccc(Cl)c1</smiles>

The title compound was prepared according to general procedure $\mathrm{D}$, from 3-chlorobenzne sulfonyl chloride ( $253 \mathrm{mg}, 1.2 \mathrm{mmol}$ ) Following conversion to the product and column chromatography (1:9 EtOAc:hexane) afforded $3 \mathrm{e}$ as a white solid (255 mg, 83\%)

${ }^{1} \mathrm{H}$ NMR $\left(400 \mathrm{MHz}, \mathrm{CDCl}_{3}\right) \delta 7.90(\mathrm{t}, J=1.9 \mathrm{~Hz}, 1 \mathrm{H}), 7.83-7.75(\mathrm{~m}, 1 \mathrm{H}), 7.55-7.48(\mathrm{~m}, 1 \mathrm{H})$, $7.42(\mathrm{t}, J=7.9 \mathrm{~Hz}, 1 \mathrm{H}), 4.85(\mathrm{~s}, 1 \mathrm{H}), 2.11(\mathrm{~s}, 1 \mathrm{H}), 1.56(\mathrm{~s}, 6 \mathrm{H})$.

${ }^{13} \mathrm{C}$ NMR $\left(101 \mathrm{MHz}, \mathrm{CDCl}_{3}\right) \delta 143.2,134.8,132.7,130.1,127.9,125.8,85.0,71.8,50.3,30.9$.

Data in accordance with literature. ${ }^{5}$

\section{5-chloro-2-methoxy-N-(2-methylbut-3-yn-2-yl)benzene-1-sulfonamide (3f)}<smiles>C#CC(C)(C)NS(=O)(=O)c1cc(Cl)ccc1OC</smiles>

The title compound was prepared according to general procedure $D$, from 2-methoxy-5chlorobenzene sulfonyl chloride ( $286 \mathrm{mg}, 1.2 \mathrm{mmol}$ ) Following conversion to the product and column chromatography (1:9 EtOAc:hexane) afforded $3 \mathbf{f}$ as a yellow solid (203 $\mathrm{mg}, 59 \%)$

${ }^{1} \mathrm{H}$ NMR $\left(400 \mathrm{MHz}, \mathrm{CDCl}_{3}\right) \delta 7.89(\mathrm{~d}, J=2.6 \mathrm{~Hz}, 1 \mathrm{H}), 7.46(\mathrm{dd}, J=8.8,2.7 \mathrm{~Hz}, 1 \mathrm{H}), 7.25$ (s, $1 \mathrm{H}), 6.93(\mathrm{~d}, J=8.8 \mathrm{~Hz}, 1 \mathrm{H}), 5.12(\mathrm{~s}, 1 \mathrm{H}), 3.97(\mathrm{~s}, 3 \mathrm{H}), 1.91(\mathrm{~s}, 1 \mathrm{H}), 1.55(\mathrm{~s}, 6 \mathrm{H})$.

${ }^{13} \mathrm{C}$ NMR $\left(101 \mathrm{MHz}, \mathrm{CDCl}_{3}\right) \delta 154.9,133.9,130.4,130.1,125.8,113.33,84.5,71.2,56.8$, 49.9, 31.0 .

Data in accordance with literature. ${ }^{5}$ 


\section{$\underline{N-(2-m e t h y l b u t-3-y n-2-y l) a c e t a m i d e ~(3 g)}$}<smiles>C#CC(C)(C)NC(C)=O</smiles>

The title compound was prepared according to general procedure $\mathrm{D}$, from acetic anhydride (122 $\mathrm{mg}, 1.2 \mathrm{mmol}$ ) Following conversion to the product and column chromatography (1:9 EtOAc:hexane) afforded $\mathbf{3 g}$ as a white solid (126 $\mathrm{mg}, 84 \%)$

${ }^{1} \mathrm{H} \mathrm{NMR}\left(400 \mathrm{MHz}, \mathrm{CDCl}_{3}\right) \delta 5.72(\mathrm{~s}, 1 \mathrm{H}), 2.31(\mathrm{~s}, 1 \mathrm{H}), 1.93(\mathrm{~d}, J=1.8 \mathrm{~Hz}, 3 \mathrm{H}), 1.61$ (d, $J=$ $2.8 \mathrm{~Hz}, 6 \mathrm{H})$.

${ }^{13} \mathrm{C}$ NMR $\left(101 \mathrm{MHz}, \mathrm{CDCl}_{3}\right) \delta 169.4,87.3,69.1,47.6,29.1,24.2$.

Data in accordance with literature. ${ }^{6}$

\section{$\underline{N \text {-(2-methylbut-3-yn-2-yl)furan-2-carboxamide (3h) }}$}<smiles>C#CC(C)(C)NC(=O)c1ccco1</smiles>

The title compound was prepared according to general procedure $D$, from 2-furoyl chloride (156 mg, $1.2 \mathrm{mmol})$ Following conversion to the product and column chromatography (1:9 EtOAc:hexane) afforded $3 \mathrm{~h}$ as a white solid (191 mg, 90\%)

${ }^{1} \mathrm{H}$ NMR $\left(400 \mathrm{MHz}, \mathrm{CDCl}_{3}\right) \delta 7.55(\mathrm{~s}, 1 \mathrm{H}), 7.39(\mathrm{~d}, \mathrm{~J}=8.4 \mathrm{~Hz}, 1 \mathrm{H}), 6.62(\mathrm{~s}, 1 \mathrm{H}), 6.59-6.50$ $(\mathrm{m}, 1 \mathrm{H}), 2.53(\mathrm{~s}, 1 \mathrm{H}), 1.89(\mathrm{~s}, 6 \mathrm{H})$.

${ }^{13} \mathrm{C}$ NMR $\left(101 \mathrm{MHz}, \mathrm{CDCl}_{3}\right) \delta 157.6,148.3,143.9,114.6,112.5,87.0,69.7,47.9,29.4$.

Data in accordance with literature. ${ }^{6}$ 


\section{2-chloro-N-(2-methylbut-3-yn-2-yl)benzamide (3i)}<smiles>C#CC(C)(C)NC(=O)c1ccccc1Cl</smiles>

The title compound was prepared according to general procedure $D$, from 2-chlorobenzoyl chloride (210 ng, $1.2 \mathrm{mmol}$ ) Following conversion to the product and column chromatography (1:9 EtOAc:hexane) afforded $3 \mathbf{i}$ as a white solid $(209 \mathrm{mg}, 79 \%)$

${ }^{1} \mathrm{H}$ NMR $\left(400 \mathrm{MHz}, \mathrm{CDCl}_{3}\right) \delta 7.62$ (dd, $\left.J=7.3,1.9 \mathrm{~Hz}, 1 \mathrm{H}\right), 7.42-7.37(\mathrm{~m}, 1 \mathrm{H}), 7.37-7.29$ $(\mathrm{m}, 2 \mathrm{H}), 5.11(\mathrm{~s}, 1 \mathrm{H}), 2.27(\mathrm{~s}, 1 \mathrm{H}), 1.62(\mathrm{~s}, 6 \mathrm{H})$.

${ }^{13} \mathrm{C}$ NMR $\left(101 \mathrm{MHz}, \mathrm{CDCl}_{3}\right) \delta 165.5,135.3,133.4,131.4,130.6,130.2,127.2,86.8,69.7$, 48.5, 29.0 .

Data in accordance with literature. ${ }^{7}$

\section{tert-butyl (2-methylbut-3-yn-2-yl)carbamate (3k)}<smiles>C#CC(C)(C)NC(=O)OC(C)(C)C</smiles>

The title compound was prepared according to general procedure $\mathrm{D}$, from di-tert butyl carbonate (261 $\mathrm{mg}, 1.2 \mathrm{mmol}$ ) Following conversion to the product and column chromatography (1:9 EtOAc:hexane) afforded $\mathbf{3 k}$ as a white solid (175 mg, 80\%)

${ }^{1} \mathrm{H}$ NMR (400 MHz, $\left.\mathrm{CDCl}_{3}\right) \delta 4.68$ (s, 1H), 2.28 (s, 1H), 1.56 (s, 6H), 1.43 (s, 9H).

${ }^{13} \mathrm{C}$ NMR $\left(101 \mathrm{MHz}, \mathrm{CDCl}_{3}\right) \delta 154.2,87.5,79.8,68.8,47.0,29.5,28.5$.

Data in accordance with literature. ${ }^{8}$ 
(4b,8a,9,9a-tetrahydro-4aH-fluoren-9-yl)methyl (2-methylbut-3-yn-2-

yl)carbamate (3I)

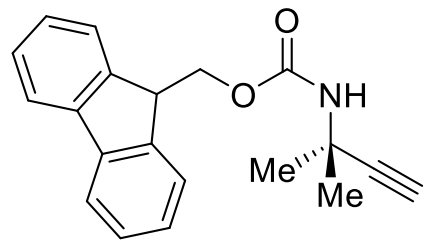

The title compound was prepared according to general procedure $\mathrm{D}$, from fluorenylmethyloxycarbonyl chloride (309 $\mathrm{mg}, 1.2 \mathrm{mmol}$ ) Following conversion to the product and column chromatography (1:9 EtOAc:hexane) afforded $\mathbf{3 l}$ as a white solid (270 $\mathrm{mg}, 74 \%)$

${ }^{1} \mathrm{H} \mathrm{NMR}\left(400 \mathrm{MHz}, \mathrm{CDCl}_{3}\right) \delta 7.77(\mathrm{~d}, J=7.5 \mathrm{~Hz}, 2 \mathrm{H}), 7.62(\mathrm{~d}, J=7.4 \mathrm{~Hz}, 2 \mathrm{H}), 7.44-7.37(\mathrm{~m}$, $2 \mathrm{H}), 7.32(\mathrm{td}, J=7.4,1.1 \mathrm{~Hz}, 2 \mathrm{H}), 5.00(\mathrm{~s}, 1 \mathrm{H}), 4.43(\mathrm{~d}, \mathrm{~J}=4.1 \mathrm{~Hz}, 2 \mathrm{H}), 4.23(\mathrm{t}, J=6.7 \mathrm{~Hz}, 1 \mathrm{H})$, $2.34(\mathrm{~s}, 1 \mathrm{H}), 1.57(\mathrm{~S}, 6 \mathrm{H})$

${ }^{13} \mathrm{C}$ NMR $\left(101 \mathrm{MHz}, \mathrm{CDCl}_{3}\right) \delta 154.6,144.1,141.4,127.8,127.1,125.1,120.1,87.1,69.3$, $66.4,47.3,29.4,27.0$.

Data in accordance with literature..$^{9}$ 


\section{Platinum Catalysed Hydrosilylation of Propargylic Amines}

\section{$\underline{\text { (2E)-N-benzyl-3-[dimethyl(phenyl)silyl]-1-phenylprop-2-en-1-amine (2a) }}$}<smiles>C(=CC(NCc1ccccc1)c1ccccc1)c1ccccc1</smiles>

The title compound was prepared according to general procedure $\mathrm{E}$, from N-benzyl-1phenylprop-2-yn-1-amine (50 mg, $0.23 \mathrm{mmol}$ ) and dimethylphenylsilane $(46.2 \mathrm{mg}, 0.33 \mathrm{mmol})$ using $\mathrm{PtCl}_{2}(0.6 \mathrm{mg}, 2.3 \mu \mathrm{mol})$ and XantPhos $(1.3 \mathrm{mg}, 2.3 \mu \mathrm{mol})$ which following conversion to the vinyl silane and column chromatography (7:3 Hexane/DCM) afforded 2a (61 mg, $70 \%$ ) as a yellow oil.

$\mathrm{R}_{\mathrm{f}}(7: 3$ Hexane/EtOAc): 0.44

IR umax $\left(\mathrm{cm}^{-1}\right)$ 3083, 3064, 3024, 2954, 2900

HRMS (ESI) m/z: [M + H]+ Calcd for $\mathrm{C}_{24} \mathrm{H}_{27} \mathrm{NSi} 358.1991$; Found 358.1987

${ }^{1} \mathrm{H}$ NMR $\left(400 \mathrm{MHz}, \mathrm{CDCl}_{3}\right) \delta 7.57-7.49(\mathrm{~m}, 2 \mathrm{H}), 7.39(\mathrm{~d}, J=5.2 \mathrm{~Hz}, 2 \mathrm{H}), 7.37(\mathrm{t}, J=2.3$ $\mathrm{Hz}, 4 \mathrm{H}), 7.35(\mathrm{dd}, J=3.6,2.1 \mathrm{~Hz}, 2 \mathrm{H}), 7.33(\mathrm{~d}, J=2.9 \mathrm{~Hz}, 3 \mathrm{H}), 7.32-7.30(\mathrm{~m}, 1 \mathrm{H}), 7.29-$ $7.27(\mathrm{~m}, 1 \mathrm{H}), 6.24(\mathrm{dd}, J=18.5,6.3 \mathrm{~Hz}, 1 \mathrm{H}), 6.03(\mathrm{dd}, J=18.6,1.0 \mathrm{~Hz}, 1 \mathrm{H}), 4.30(\mathrm{dd}, J=$ 6.3, 1.0 Hz, 1H), $3.79-3.70(\mathrm{~m}, 2 \mathrm{H}), 1.69(\mathrm{~s}, 1 \mathrm{H}), 0.35(\mathrm{~d}, J=4.0 \mathrm{~Hz}, 6 \mathrm{H})$.

${ }^{13} \mathrm{C}$ NMR $\left(101 \mathrm{MHz}, \mathrm{CDCl}_{3}\right) \delta 149.9,142.6,140.5,138.8,133.9,129.0,128.6,128.5,128.3$, $128.1,127.8,127.6,127.3,127.0,67.3,51.5,-2.5$.

\section{$\underline{\mathrm{N}}-\{(2 \mathrm{E})-3-[$ dimethyl(phenyl)silyl]-1-phenylprop-2-en-1-yl\}aniline (2b)}<smiles>CC(C=CC(Nc1ccccc1)c1ccccc1)c1ccccc1</smiles>

The title compound was prepared according to general procedure $\mathrm{E}$ from $\mathrm{N}$-[2-phenylbut-3yn-2-yl]aniline (50 mg, $0.21 \mathrm{mmol}$ ) and dimethylphenylsilane (43.4 mg, $0.32 \mathrm{mmol}$ ) using $\mathrm{PtCl}_{2}$ $(0.6 \mathrm{mg}, 2.1 \mu \mathrm{mol})$ and XantPhos $(1.2 \mathrm{mg}, 2.1 \mu \mathrm{mol})$ which following conversion to the vinyl silane and column chromatography (7:3 Hexane/DCM) afforded $2 \mathrm{~b}$ (59 $\mathrm{mg}, 82 \%)$ as a colourless oil.

$\mathrm{R}_{\mathrm{f}}(7: 3$ Hexane/DCM): 0.44

IR vmax $\left(\mathrm{cm}^{-1}\right)$ 3413, 3051, 2957, 2845, 1600, 1501

HRMS (ESI) m/z: [M + H]+ Calcd for $\mathrm{C}_{23} \mathrm{H}_{25} \mathrm{NSi} 344.1835$; Found 344.1828 
${ }^{1} \mathrm{H}$ NMR $\left(400 \mathrm{MHz}, \mathrm{CDCl}_{3}\right) \delta 7.45(\mathrm{~d}, J=7.5 \mathrm{~Hz}, 2 \mathrm{H}), 7.33(\mathrm{t}, J=6.5 \mathrm{~Hz}, 7 \mathrm{H}), 7.29-7.26(\mathrm{~m}$, $1 \mathrm{H}), 7.12(\mathrm{t}, J=7.9 \mathrm{~Hz}, 2 \mathrm{H}), 6.69(\mathrm{~d}, J=7.3 \mathrm{~Hz}, 1 \mathrm{H}), 6.58(\mathrm{~d}, J=8.0 \mathrm{~Hz}, 2 \mathrm{H}), 6.26(\mathrm{dd}, J=$ 18.6, $5.1 \mathrm{~Hz}, 1 \mathrm{H}), 6.07(\mathrm{~d}, J=18.6 \mathrm{~Hz}, 1 \mathrm{H}), 4.95(\mathrm{~s}, 1 \mathrm{H}), 4.06(\mathrm{~s}, 1 \mathrm{H}), 0.32(\mathrm{~s}, 6 \mathrm{H})$.

${ }^{13} \mathrm{C}$ NMR $\left(101 \mathrm{MHz}, \mathrm{CDCl}_{3}\right) \delta 147.9,147.3,141.9,138.6,134.0,129.2,129.1,128.9,128.7$, $127.9,127.5,127.4,117.7,113.7,62.8,-2.4,-2.5$.

\section{(3E)-N-benzyl-4-[dimethyl(phenyl)silyl]-2-(2-fluorophenyl)but-3-en-2-amine (2c)}<smiles>CC(/C=C/Sc1ccccc1)(NCc1ccccc1)c1ccccc1F</smiles>

The title compound was prepared according to general procedure $\mathrm{E}$, from N-[2-(2fluorophenyl)but-3-yn-2-yl]aniline (50 mg, $0.2 \mathrm{mmol}$ ) and dimethylphenylsilane (41 $\mathrm{mg}, 0.3$ mmol) using $\mathrm{PtCl}_{2}(0.52 \mathrm{mg}, 2 \mu \mathrm{mol})$ and XantPhos $(1.2 \mathrm{mg}, 2 \mu \mathrm{mol})$ which following conversion to the vinyl silane and column chromatography (7:3 Hexane/DCM) afforded 2c (63 $\mathrm{mg}, 85 \%)$ as a colourless oil.

$\mathrm{R}_{\mathrm{f}}(7: 3$ Hexane/DCM): 0.26

IR umax $\left(\mathrm{cm}^{-1}\right)$ 3066, 3026, 2956, 2900, 2844

HRMS (ESI) m/z: [M + H]+ Calcd for $\mathrm{C}_{25} \mathrm{H}_{28} \mathrm{NFSi} 390.2053$; Found 390.2058

${ }^{1} \mathrm{H}$ NMR $\left(400 \mathrm{MHz}, \mathrm{CDCl}_{3}\right) \delta 7.50(\mathrm{td}, J=6.1,2.0 \mathrm{~Hz}, 2 \mathrm{H}), 7.45(\mathrm{dd}, J=8.0,1.6 \mathrm{~Hz}, 1 \mathrm{H}), 7.37$ $-7.32(\mathrm{~m}, 3 \mathrm{H}), 7.29(\mathrm{t}, J=6.0 \mathrm{~Hz}, 3 \mathrm{H}), 7.24(\mathrm{dd}, J=9.0,4.7 \mathrm{~Hz}, 3 \mathrm{H}), 7.13(\mathrm{t}, J=7.2 \mathrm{~Hz}, 1 \mathrm{H})$, 7.05 (dd, $J=12.3,8.2 \mathrm{~Hz}, 1 \mathrm{H}), 6.39$ (d, $J=19.0 \mathrm{~Hz}, 1 \mathrm{H}), 5.89$ (d, $J=18.9 \mathrm{~Hz}, 1 \mathrm{H}), 3.52$ (dd, $J=39.3,12.2 \mathrm{~Hz}, 2 \mathrm{H}), 2.00(\mathrm{~s}, 1 \mathrm{H}), 1.65(\mathrm{~s}, 3 \mathrm{H}), 0.34(\mathrm{~d}, J=3.1 \mathrm{~Hz}, 6 \mathrm{H})$

${ }^{13} \mathrm{C}$ NMR $\left(101 \mathrm{MHz}, \mathrm{CDCl}_{3}\right) \delta 161.3(\mathrm{~d}, J=247.7 \mathrm{~Hz}), 153.0,141.0,139.0,134.0,132.7$, $129.0,128.9,128.5,128.4,127.8,127.0,124.7,124.0,116.5,116.3,61.6,48.0,25.4,-2.3$.

\section{(1E)-N-benzyl-1-[dimethyl(phenyl)silyl]-3,4,4-trimethylpent-1-en-3-amine (2d)}<smiles>CC(C)/C=C/[C@H](NCc1ccccc1)C(C)(C)C</smiles>

The title compound was prepared according to general procedure $\mathrm{E}$, from N-benzyl-3,4,4trimethylpent-1-yn-3-amine $(50 \mathrm{mg}, 0.23 \mathrm{mmol}$ ) and dimethylphenylsilane $(47.5 \mathrm{mg}, 0.32$ mmol) using $\mathrm{PtCl}_{2}(0.6 \mathrm{mg}, 2.3 \mu \mathrm{mol})$ and XantPhos $(1.3 \mathrm{mg}, 2.3 \mu \mathrm{mol})$ which following conversion to the vinyl silane and column chromatography (9:1 Hexane/EtOAc) afforded $2 \mathbf{d}$ (69 $\mathrm{mg}, 86 \%)$ as a colourless oil. 
$\mathrm{R}_{\mathrm{f}}(9: 1$ Hexane/EtOAc): 0.63

IR umax $\left(\mathrm{cm}^{-1}\right) 3085,3067,2955,1247$

HRMS (ESI) m/z: [M + H]+ Calcd for $\mathrm{C}_{23} \mathrm{H}_{33} \mathrm{NSi} 352.2461$; Found 352.2455

${ }^{1} \mathrm{H}$ NMR $\left(400 \mathrm{MHz}, \mathrm{CDCl}_{3}\right) \delta 7.56-7.50(\mathrm{~m}, J=4.1,2.5 \mathrm{~Hz}, 2 \mathrm{H}), 7.36-7.28(\mathrm{~m}, J=18.4$, 9.5, 4.8 Hz, 7H), $7.24-7.20(\mathrm{~m}, 1 \mathrm{H}), 6.10(\mathrm{~d}, J=19.3 \mathrm{~Hz}, 1 \mathrm{H}), 5.78(\mathrm{~d}, J=19.4 \mathrm{~Hz}, 1 \mathrm{H}), 3.54$ $(\mathrm{dd}, J=36.7,12.8 \mathrm{~Hz}, 2 \mathrm{H}), 1.16(\mathrm{~s}, 3 \mathrm{H}), 0.91(\mathrm{~s}, 9 \mathrm{H}), 0.34(\mathrm{~s}, 6 \mathrm{H})$.

${ }^{13} \mathrm{C}$ NMR $\left(101 \mathrm{MHz}, \mathrm{CDCl}_{3}\right) \delta 153.1,142.5,139.4,134.0,129.0,128.4,128.2,127.9,127.6$, $126.7,63.3,47.7,37.5,25.6,17.5,-2.1$.

\section{N-benzyl-1-\{(E)-2-[di methyl(phenyl)silyl]ethenyl\}cyclohexan-1-amine (2e)}<smiles>C(/C=C/C1(NCc2ccccc2)CCCCC1)=C\c1ccccc1</smiles>

The title compound was prepared according to general procedure $\mathrm{E}$, from N-benzyl-1ethynylcyclohexan-1-amine $(50 \mathrm{mg}, 0.23 \mathrm{mmol})$ and dimethylphenylsilane $(47.9 \mathrm{mg}, 0.35$ mmol) using $\mathrm{PtCl}_{2}(0.6 \mathrm{mg}, 2.3 \mu \mathrm{mol})$ and XantPhos $(1.4 \mathrm{mg}, 2.3 \mu \mathrm{mol})$ which following conversion to the vinyl silane and column chromatography (9:1 Hexane/EtOAc) afforded $2 e$ $(81,9 \mathrm{mg}, 77 \%)$ as a colourless oil.

$R_{f}(9: 1$ Hexane/EtOAc): 0.61

IR umax $\left(\mathrm{cm}^{-1}\right)$ 3069, 3026, 2956, 2940, 2898

HRMS (ESI) m/z: [M + H] + Calcd for $\mathrm{C}_{23} \mathrm{H}_{31} \mathrm{NSi} 350.2304$; Found 350.2301

${ }^{1} \mathrm{H}$ NMR $\left(400 \mathrm{MHz}, \mathrm{CDCl}_{3}\right) \delta 7.55(\mathrm{dd}, J=6.5,3.0 \mathrm{~Hz}, 2 \mathrm{H}), 7.39-7.33(\mathrm{~m}, 3 \mathrm{H}), 7.30(\mathrm{~d}, J=$ $4.4 \mathrm{~Hz}, 4 \mathrm{H}), 7.25-7.21(\mathrm{~m}, 1 \mathrm{H}), 5.92(\mathrm{q}, J=19.3 \mathrm{~Hz}, 4 \mathrm{H}), 3.53(\mathrm{~s}, 1 \mathrm{H}), 1.77-1.51(\mathrm{~m}, 6 \mathrm{H})$, $1.44(\mathrm{dd}, J=13.9,6.9 \mathrm{~Hz}, 4 \mathrm{H}), 0.37(\mathrm{~s}, 6 \mathrm{H})$.

${ }^{13} \mathrm{C}$ NMR $\left(101 \mathrm{MHz}, \mathrm{CDCl}_{3}\right) \delta 154.8,141.7,139.4,134.0,129.0,128.5,127.9,126.8,125.4$, 57.5, 46.5, 35.4, 26.3, 22.2, -2.1. 


\section{(1E)-N-benzyl-1-[dimethyl(phenyl)silyl]-3,5-dimethylhex-1-en-3-amine (2f)}<smiles>CC(C)CC(C)(/C=C/Sc1ccccc1)NCc1ccccc1</smiles>

The title compound was prepared according to general procedure $\mathrm{E}$, from N-benzyl-3,5dimethylhex-1-yn-3-amine $(75 \mathrm{mg}, 0.35 \mathrm{mmol})$ and dimethylphenylsilane $(71.2 \mathrm{mg}, 0.53$ mmol) using $\mathrm{PtCl}_{2}(0.9 \mathrm{mg}, 3.5 \mu \mathrm{mol})$ and XantPhos $(2 \mathrm{mg}, 3.5 \mu \mathrm{mol})$ which following conversion to the vinyl silane and column chromatography (9:1 Hexane/EtOAc) afforded $\mathbf{2 f}$ (122 $\mathrm{mg}, 79 \%$ ) as a colourless oil.

$R_{f}(9: 1$ Hexane/EtOAc): 0.48

IR umax $\left(\mathrm{cm}^{-1}\right)$ 3067, 3026, 2954, 2868, 1606

HRMS (ESI) m/z: [M + H]+ Calcd for $\mathrm{C}_{23} \mathrm{H}_{33} \mathrm{NSi} 352.2461$; Found 352.2455

${ }^{1} \mathrm{H} \mathrm{NMR}\left(400 \mathrm{MHz}, \mathrm{CDCl}_{3}\right) \delta 7.54$ (dd, $\left.J=6.4,3.1 \mathrm{~Hz}, 2 \mathrm{H}\right), 7.35$ (dd, $\left.J=4.6,1.9 \mathrm{~Hz}, 3 \mathrm{H}\right)$, $7.30(\mathrm{~d}, J=4.4 \mathrm{~Hz}, 4 \mathrm{H}), 7.25-7.19(\mathrm{~m}, 1 \mathrm{H}), 6.03(\mathrm{~d}, J=19.1 \mathrm{~Hz}, 1 \mathrm{H}), 5.83(\mathrm{~d}, J=19.2 \mathrm{~Hz}$, $1 \mathrm{H}), 3.64-3.55(\mathrm{~m}, 2 \mathrm{H}), 1.71(\mathrm{dt}, J=12.3,5.9 \mathrm{~Hz}, 1 \mathrm{H}), 1.48-1.38(\mathrm{~m}, 3 \mathrm{H}), 1.23(\mathrm{~s}, 3 \mathrm{H})$, $0.91(\mathrm{dd}, J=9.0,6.7 \mathrm{~Hz}, 6 \mathrm{H}), 0.41(\mathrm{~s}, 1 \mathrm{H}), 0.35(\mathrm{~s}, 6 \mathrm{H})$.

${ }^{13} \mathrm{C} \mathrm{NMR}\left(101 \mathrm{MHz}, \mathrm{CDCl}_{3}\right) \delta 155.8,141.6,139.2,134.0,129.0,128.5,128.4,127.9,126.9$, $124.8,59.0,50.2,47.2,25.3,25.1,24.2,22.8,-2.2$.

\section{$\underline{N}-\{(3 E)-4-[$ dimethyl(phenyl)silyl]-2-phenylbut-3-en-2-yl\}aniline (2g)}<smiles>CC(/C=C/c1ccccc1)(Nc1ccccc1)c1ccccc1</smiles>

The title compound was prepared according to general procedure $\mathrm{E}$, from N-[2-phenylbut-3yn-2-yl]aniline (50 mg, $0.23 \mathrm{mmol}$ ) and dimethylphenylsilane (46.2 mg, $0.34 \mathrm{mmol}$ ) using $\mathrm{PtCl}_{2}$ $(0.6 \mathrm{mg}, 2.3 \mu \mathrm{mol})$ and XantPhos $(1.3 \mathrm{mg}, 2.3 \mu \mathrm{mol})$ which following conversion to the vinyl silane and column chromatography (7:3 Hexane/DCM) afforded $\mathbf{2 g}(69 \mathrm{mg}, 85 \%)$ as a colourless oil.

$\mathrm{R}_{\mathrm{f}}(7: 3$ Hexane/DCM): 0.60

IR umax $\left(\mathrm{cm}^{-1}\right)$ 3411, 3053, 3016, 2960, 1599

HRMS (ESI) m/z: [M + H]+ Calcd for $\mathrm{C}_{24} \mathrm{H}_{28} \mathrm{NSi}$ 358.1991; Found 358.1985

${ }^{1} \mathrm{H}$ NMR (400 MHz, CDCl 3 ) $\delta 7.47(\mathrm{~d}, J=7.4 \mathrm{~Hz}, 2 \mathrm{H}), 7.43-7.39(\mathrm{~m}, 2 \mathrm{H}), 7.32(\mathrm{t}, J=7.7 \mathrm{~Hz}$, $5 \mathrm{H}), 7.22(\mathrm{~d}, J=7.6 \mathrm{~Hz}, 1 \mathrm{H}), 7.00(\mathrm{t}, J=7.9 \mathrm{~Hz}, 2 \mathrm{H}), 6.62(\mathrm{t}, J=7.3 \mathrm{~Hz}, 1 \mathrm{H}), 6.53(\mathrm{~d}, J=18.9$ $\mathrm{Hz}, 1 \mathrm{H}), 6.40(\mathrm{~d}, J=8.6 \mathrm{~Hz}, 2 \mathrm{H}), 6.01(\mathrm{~d}, J=18.8 \mathrm{~Hz}, 1 \mathrm{H}), 4.17(\mathrm{~s}, 1 \mathrm{H}), 1.68(\mathrm{~s}, 3 \mathrm{H}), 0.31(\mathrm{~d}$, $J=1.2 \mathrm{~Hz}, 6 \mathrm{H})$.

${ }^{13} \mathrm{C}$ NMR $\left(101 \mathrm{MHz}, \mathrm{CDCl}_{3}\right) \delta 152.1,145.8,145.4,138.8,134.0,129.1,128.7,128.6,127.9$, $126.8,126.6,126.0,117.4,116.0,61.7,29.4,-2.4$. 


\section{$\mathrm{N}$-[(3E)-4-[dimethyl(phenyl)silyl]-2-(4-methylphenyl)but-3-en-2-yl]aniline (2h)}<smiles>Cc1ccc(C(C)(/C=C/[AsH2]c2ccccc2)Nc2ccccc2)cc1</smiles>

The title compound was prepared according to general procedure $\mathrm{E}$, from N-[2-(4methylphenyl)but-3-yn-2-yl]aniline $(50 \mathrm{mg}, 0.21 \mathrm{mmol})$ and dimethylphenylsilane $(43.4 \mathrm{mg}$, $0.32 \mathrm{mmol})$ using $\mathrm{PtCl}_{2}(0.6 \mathrm{mg}, 2.1 \mu \mathrm{mol})$ and XantPhos $(1.2 \mathrm{mg}, 2.1 \mu \mathrm{mol})$ which following conversion to the vinyl silane and column chromatography (7:3 Hexane/DCM) afforded $\mathbf{2} \mathbf{h}$ (70 $\mathrm{mg}, 89 \%$ ) as a colourless oil.

$\mathrm{R}_{\mathrm{f}}(7: 3$ Hexane/DCM $) 0.54$

IR umax $\left(\mathrm{cm}^{-1}\right)$ 3409, 3018, 2973, 1602, 1501

HRMS (ESI) m/z: [M + H] + Calcd for $\mathrm{C}_{25} \mathrm{H}_{29} \mathrm{NSi} 372.2148$; Found 372.2140

${ }^{1} \mathrm{H}$ NMR $\left(400 \mathrm{MHz}, \mathrm{CDCl}_{3}\right) \delta 7.47-7.41(\mathrm{~m}, 2 \mathrm{H}), 7.39-7.31(\mathrm{~m}, 5 \mathrm{H}), 7.15(\mathrm{~d}, J=8.0 \mathrm{~Hz}$, 2H), $7.02(\mathrm{t}, J=7.8 \mathrm{~Hz}, 2 \mathrm{H}), 6.64(\mathrm{t}, J=7.3 \mathrm{~Hz}, 1 \mathrm{H}), 6.54(\mathrm{~d}, J=18.8 \mathrm{~Hz}, 1 \mathrm{H}), 6.44(\mathrm{~d}, J=$ $8.5 \mathrm{~Hz}, 2 \mathrm{H}), 6.02(\mathrm{~d}, J=18.8 \mathrm{~Hz}, 1 \mathrm{H}), 4.17(\mathrm{~s}, 1 \mathrm{H}), 2.35(\mathrm{~s}, 3 \mathrm{H}), 1.69(\mathrm{~s}, 3 \mathrm{H}), 0.33(\mathrm{~s}, 6 \mathrm{H})$.

${ }^{13} \mathrm{C}$ NMR $\left(101 \mathrm{MHz}, \mathrm{CDCl}_{3}\right) \delta 152.3,146.0,142.4,138.8,136.3,134.0,129.3,129.1,128.7$, $127.9,126.5,125.8,117.4,116.0,61.5,29.4,21.1,-2.3,-2.4$.

\section{$\mathrm{N}-\{(1 \mathrm{E})-1-[$ dimethyl(phenyl)silyl]-3,5-dimethylhex-1-en-3-yl\}-4-} (trifluoromethyl)aniline (2i)<smiles>CC(C)CC(C)(/C=C/Sc1ccccc1)Nc1ccc(C(F)(F)F)cc1</smiles>

The title compound was prepared according to general procedure $\mathrm{E}$, from $\mathrm{N}$-(3,5-dimethylhex1-yn-3-yl)-4-(trifluoromethyl)aniline $(50 \mathrm{mg}, 0.21 \mathrm{mmol})$ and dimethylphenylsilane $(43.4 \mathrm{mg}$, $0.32 \mathrm{mmol})$ using $\mathrm{PtCl}_{2}(0.6 \mathrm{mg}, 2.1 \mu \mathrm{mol})$ and XantPhos (1.2 mg, $\left.2.1 \mu \mathrm{mol}\right)$ which following conversion to the vinyl silane and column chromatography (7:3 Hexane/DCM) afforded $\mathbf{2} \mathbf{i}(70$ $\mathrm{mg}, 89 \%)$ as a colourless oil.

$\mathrm{R}_{\mathrm{f}}(7: 3$ Hexane:DCM, 0.48)

HRMS (ESI) m/z: [M + H] + Calcd for $\mathrm{C}_{23} \mathrm{H}_{30} \mathrm{~F}_{3} \mathrm{NSi} 406.2178$; Found 406.2177

IR umax $\left(\mathrm{cm}^{-1}\right)$ 3067, 3022, 2955, 2869, 1614

${ }^{1} \mathrm{H}$ NMR $\left(400 \mathrm{MHz}, \mathrm{CDCl}_{3}\right) \delta 7.50-7.45(\mathrm{~m}, 2 \mathrm{H}), 7.38-7.31(\mathrm{~m}, 3 \mathrm{H}), 7.28(\mathrm{~d}, \mathrm{~J}=8.6 \mathrm{~Hz}$, $2 \mathrm{H}), 6.61(\mathrm{~d}, J=8.5 \mathrm{~Hz}, 2 \mathrm{H}), 6.14(\mathrm{~d}, J=19.2 \mathrm{~Hz}, 1 \mathrm{H}), 5.93(\mathrm{~d}, J=19.2 \mathrm{~Hz}, 1 \mathrm{H}), 4.09(\mathrm{~s}, 1 \mathrm{H})$, $1.83-1.71(\mathrm{~m}, 1 \mathrm{H}), 1.60(\mathrm{dt}, J=14.1,7.8 \mathrm{~Hz}, 2 \mathrm{H}), 1.40(\mathrm{~s}, 3 \mathrm{H}), 0.92(\mathrm{dd}, J=22.3,6.7 \mathrm{~Hz}$, $6 \mathrm{H}), 0.34(\mathrm{~d}, J=1.5 \mathrm{~Hz}, 6 \mathrm{H})$.

${ }^{13} \mathrm{C}$ NMR $\left(101 \mathrm{MHz}, \mathrm{CDCl}_{3}\right) \delta 153.5,149.1,138.6,133.9,129.1,127.9,126.5,126.1,126.0$, $118.3(\mathrm{q}, J=32.8 \mathrm{~Hz}), 114.3,59.1,50.3,25.1,24.9,24.7,24.0,-2.5$. 
${ }^{19} \mathrm{~F}$ NMR $\left(376 \mathrm{MHz}, \mathrm{CDCl}_{3}\right) \delta-60.84$.

\section{2-chloro-N-\{(2E)-3-[dimethyl(phenyl)silyl]-1-phenylprop-2-en-1-yl\}-4-}

\section{methylaniline (2i)}<smiles>Cc1ccc(NC(/C=C/Sc2ccccc2)c2ccccc2)c(Cl)c1</smiles>

The title compound was prepared according to general procedure $\mathrm{E}$, from 2-chloro-4-methyl$\mathrm{N}$-(1-phenylprop-2-yn-1-yl)aniline $(50 \mathrm{mg}, 0.19 \mathrm{mmol})$ and dimethylphenylsilane $(40 \mathrm{mg}, 0.29$ mmol) using $\mathrm{PtCl}_{2}(1.1 \mathrm{mg}, 1.9 \mu \mathrm{mol})$ and XantPhos $(2.3 \mathrm{mg}, 1.9 \mu \mathrm{mol})$ which following conversion to the vinyl silane and column chromatography (9:1 Hexane/EtOAc) afforded $\mathbf{2 j}$ (58 $\mathrm{mg}, 76 \%)$ as a yellow oil.

$\mathrm{R}_{\mathrm{f}}(9: 1$ Hexane/EtOAc) 0.36

HRMS (ESI) m/z: [M + H]+ Calcd for $\mathrm{C}_{24} \mathrm{H}_{26} \mathrm{CINSi} 392.1601$; Found 392.1607

IR umax $\left(\mathrm{cm}^{-1}\right) 3066,3026,2954,2920$

${ }^{1} \mathrm{H}$ NMR $\left(400 \mathrm{MHz}, \mathrm{CDCl}_{3}\right) \delta 7.48-7.43(\mathrm{~m}, 2 \mathrm{H}), 7.35-7.32(\mathrm{~m}, 6 \mathrm{H}), 7.31-7.26(\mathrm{~m}, 2 \mathrm{H})$, 7.08 (d, $J=1.6 \mathrm{~Hz}, 1 \mathrm{H}), 6.83(\mathrm{dd}, J=8.2,1.5 \mathrm{~Hz}, 1 \mathrm{H}), 6.47(\mathrm{~d}, J=8.3 \mathrm{~Hz}, 1 \mathrm{H}), 6.28$ (dd, $J=$ 18.6, $5.1 \mathrm{~Hz}, 1 \mathrm{H}), 6.05(\mathrm{dd}, J=18.6,1.4 \mathrm{~Hz}, 1 \mathrm{H}), 4.97(\mathrm{dd}, J=5.0,0.8 \mathrm{~Hz}, 1 \mathrm{H}), 2.19(\mathrm{~s}, 3 \mathrm{H})$, $0.33(\mathrm{~d}, J=1.5 \mathrm{~Hz}, 6 \mathrm{H})$.

${ }^{13} \mathrm{C}$ NMR $\left(101 \mathrm{MHz}, \mathrm{CDCl}_{3}\right) \delta 147.5,141.4,140.7,138.5,133.9,129.5,129.1,128.9,128.9$, $128.2,127.9,127.6,127.3,127.2,119.2,112.9,62.7,20.2,-2.5$.

(2E)-N-[(3-chlorophenyl)methyl]-3-[dimethyl(phenyl)silyl]-1-phenylprop-2-en-1amine (2k)<smiles>Clc1cccc(CNC(/C=C/Cc2ccccc2)c2ccccc2)c1</smiles>

The title compound was prepared according to general procedure $\mathrm{E}$, from 2-chloro-4-methyl$\mathrm{N}$-[(3-chlorophenyl)methyl]-1-phenylprop-2-yn-1-amine $\quad(50 \quad \mathrm{mg}, \quad 0.19 \mathrm{mmol})$ and dimethylphenylsilane (40 mg, $0.29 \mathrm{mmol})$ using $\mathrm{PtCl}_{2}(1.1 \mathrm{mg}, 1.9 \mu \mathrm{mol})$ and XantPhos $(2.3$ $\mathrm{mg}, 1.9 \mu \mathrm{mol})$ which following conversion to the vinyl silane and column chromatography (9:1 Hexane/EtOAc) afforded 2k (58 mg, $79 \%$ ) as a yellow oil.

$\mathrm{R}_{\mathrm{f}}(19: 1$ Hexane/EtOAc): 0.39 
HRMS (ESI) m/z: [M + H] + Calcd for $\mathrm{C}_{24} \mathrm{H}_{26} \mathrm{CINSi} 392.1601$; Found 392.1593

IR umax $\left(\mathrm{cm}^{-1}\right)$ 3065, 3024, 2965, 2825

${ }^{1} \mathrm{H}$ NMR $\left(400 \mathrm{MHz}, \mathrm{CDCl}_{3}\right) \delta 7.66(\mathrm{dd}, J=11.9,7.1 \mathrm{~Hz}, 1 \mathrm{H}), 7.52-7.41(\mathrm{~m}, 3 \mathrm{H}), 7.33(\mathrm{~d}, J$ $=3.0 \mathrm{~Hz}, 7 \mathrm{H}), 7.24-7.19(\mathrm{~m}, 2 \mathrm{H}), 7.16(\mathrm{~d}, J=6.5 \mathrm{~Hz}, 1 \mathrm{H}), 6.17(\mathrm{dd}, J=18.6,6.3 \mathrm{~Hz}, 1 \mathrm{H})$, $5.99(\mathrm{~d}, J=18.6 \mathrm{~Hz}, 1 \mathrm{H}), 4.23(\mathrm{~d}, J=6.2 \mathrm{~Hz}, 1 \mathrm{H}), 3.72-3.61(\mathrm{~m}, 2 \mathrm{H}), 1.63(\mathrm{~s}, 1 \mathrm{H}), 0.31(\mathrm{~d}$, $J=4.1 \mathrm{~Hz}, 6 \mathrm{H})$.

${ }^{13} \mathrm{C} \mathrm{NMR}\left(101 \mathrm{MHz}, \mathrm{CDCl}_{3}\right) \delta 149.7,142.7,142.5,138.8,134.3,133.9,129.8,129.1,128.7$, $128.5,128.4,127.9,127.6,127.4,127.2,126.4,67.4,51.0,-2.4$.

(3E)-4-[dimethyl(phenyl)silyl]-N-[(4-methoxyphenyl)methyl]-2-methylbut-3-en-2amine (2l)<smiles>COc1ccc(CNC(C)(C)/C=C/Sc2ccccc2)cc1</smiles>

The title compound was prepared according to general procedure $\mathrm{E}$, from $\mathrm{N}-[(4-$ methoxyphenyl)methyl]-2-methylbut-3-yn-2-amine $(30 \quad \mathrm{mg}, \quad 0.148 \quad \mathrm{mmol})$ and dimethylphenylsilane $(30 \mathrm{mg}, 0.22 \mathrm{mmol})$ using $\mathrm{PtCl}_{2}(0.4 \mathrm{mg}, 1.5 \mu \mathrm{mol})$ and XantPhos $(0.9$ $\mathrm{mg}, 1.5 \mu \mathrm{mol}$ ) which following conversion to the vinyl silane and column chromatography (9:1 Hexane/EtOAc) afforded 2l (43 mg, 86\%) as a colourless oil.

$R_{f}(9: 1$ Hexane:EtOAc, 0.19)

IR umax $\left(\mathrm{cm}^{-1}\right)$ 3098, 3044, 2995, 29702825

HRMS (ESI) m/z: [M + H] + Calcd for $\mathrm{C}_{21} \mathrm{H}_{30} \mathrm{NOSi} 340.2097$; Found 340.2091

${ }^{1} \mathrm{H}$ NMR $\left(400 \mathrm{MHz}, \mathrm{CDCl}_{3}\right) \delta 7.55-7.51(\mathrm{~m}, 2 \mathrm{H}), 7.35(\mathrm{dd}, J=4.2,2.2 \mathrm{~Hz}, 3 \mathrm{H}), 7.21-7.17$ $(\mathrm{m}, 2 \mathrm{H}), 6.86-6.81(\mathrm{~m}, 2 \mathrm{H}), 6.07(\mathrm{~d}, J=19.0 \mathrm{~Hz}, 1 \mathrm{H}), 5.83(\mathrm{~d}, J=19.0 \mathrm{~Hz}, 1 \mathrm{H}), 3.78(\mathrm{~s}, 3 \mathrm{H})$, $3.53(\mathrm{~s}, 2 \mathrm{H}), 1.22(\mathrm{~s}, 6 \mathrm{H}), 0.35(\mathrm{~s}, 6 \mathrm{H})$.

${ }^{13} \mathrm{C}$ NMR $\left(101 \mathrm{MHz}, \mathrm{CDCl}_{3}\right) \delta 158.6,155.8,139.2,133.9,133.5,129.5,129.0,127.9,124.3$, $113.9,56.0,55.4,47.2,27.1,-2.2$. 


\section{Platinum Catalysed Hydrosilylation of Propargylic Amides, Carbamates and Sulfonamides}

$N-\{(3 E)-4-[d i m e t h y l(p h e n y l)$ silyl]-2-methylbut-3-en-2-yl\}-benzene-1-sulfonamide (4a)<smiles>CC(C)(C=C[AsH2+]c1ccccc1)NS(=O)(=O)c1ccccc1</smiles>

The title compound was prepared according to general procedure $\mathrm{E}$, from N-(2-methylbut-3yn-2-yl)benzenesulfonamide $(75 \mathrm{mg}, 0.31 \mathrm{mmol}$ ) and dimethylphenylsilane $(69 \mathrm{mg}, 0.51$ mmol) using $\mathrm{PtCl}_{2}(0.9 \mathrm{mg}, 3.2 \mu \mathrm{mol})$ and XantPhos $(1.8 \mathrm{mg}, 3.2 \mu \mathrm{mol})$ which following conversion to the vinyl silane and column chromatography (4:1 Hexane/EtOAc) afforded $4 a$ (94 $\mathrm{mg}, 92 \%$ ) as a white solid.

$R_{f}(4: 1$ Hexane/EtOAc): 0.28

HRMS (ESI) m/z: [M + H] + Calcd for $\mathrm{C}_{19} \mathrm{H}_{25} \mathrm{NO}_{2} \mathrm{SSi} 360.1454$; Found 360.1460

IR umax $\left(\mathrm{cm}^{-1}\right) 3275,3069,2976,2943,1613$

${ }^{1} \mathrm{H}$ NMR $\left(400 \mathrm{MHz}, \mathrm{CDCl}_{3}\right) \delta 7.83(\mathrm{~d}, J=7.5 \mathrm{~Hz}, 2 \mathrm{H}), 7.49(\mathrm{~d}, J=7.3 \mathrm{~Hz}, 1 \mathrm{H}), 7.42(\mathrm{~d}, J=7.6$ $\mathrm{Hz}, 3 \mathrm{H}), 7.40(\mathrm{~s}, 1 \mathrm{H}), 7.37-7.32(\mathrm{~m}, J=5.7 \mathrm{~Hz}, 3 \mathrm{H}), 5.94(\mathrm{~d}, J=18.9 \mathrm{~Hz}, 1 \mathrm{H}), 5.83(\mathrm{~d}, J=$ $18.9 \mathrm{~Hz}, 1 \mathrm{H}), 4.57(\mathrm{~s}, 1 \mathrm{H}), 1.31(\mathrm{~s}, 6 \mathrm{H}), 0.22(\mathrm{~s}, 6 \mathrm{H})$.

${ }^{13} \mathrm{C}$ NMR $\left(101 \mathrm{MHz}, \mathrm{CDCl}_{3}\right) \delta 151.8,143.1,138.4,133.9,132.4,129.2,129.0,128.0,127.3$, $125.4,58.7,28.1,-2.7$.

\section{$N-\{(3 E)-4-[d i m e t h y l(p h e n y l)$ silyl]-2-methylbut-3-en-2-yl\}-4-methylbenzene-1-} sulfonamide (4b)<smiles>Cc1ccc(S(=O)(=O)NC(C)(C)/C=C/CSc2ccccc2)cc1</smiles>

The title compound was prepared according to general procedure $\mathrm{E}$, from 4-methyl-N-(2methylbut-3-yn-2-yl)benzene-1-sulfonamide $(75 \mathrm{mg}, 0.31 \mathrm{mmol})$ and dimethylphenylsilane (69 mg, $0.51 \mathrm{mmol})$ using $\mathrm{PtCl}_{2}(0.9 \mathrm{mg}, 3.1 \mu \mathrm{mol})$ and XantPhos $(1.8 \mathrm{mg}, 3.1 \mu \mathrm{mol})$ which following conversion to the vinyl silane and column chromatography (4:1 Hexane/EtOAc) afforded 4b (118 $\mathrm{mg}, 84 \%)$ as a pale yellow solid.

$\mathrm{R}_{\mathrm{f}}(4: 1$ Hexane/EtOAc): 0.28

HRMS (ESI) m/z: [M + H] + Calcd for $\mathrm{C}_{20} \mathrm{H}_{27} \mathrm{NO}_{2} \mathrm{SSi} 374.1610$; Found 374.1605

IR umax $\left(\mathrm{cm}^{-1}\right) 3294,2978,1723,1699$ 
${ }^{1} \mathrm{H}$ NMR $\left(400 \mathrm{MHz}, \mathrm{CDCl}_{3}\right) \delta 7.73(\mathrm{~d}, J=8.1 \mathrm{~Hz}, 2 \mathrm{H}), 7.47-7.40(\mathrm{~m}, 2 \mathrm{H}), 7.35(\mathrm{dd}, J=5.6$, $1.1 \mathrm{~Hz}, 3 \mathrm{H}), 7.21(\mathrm{~d}, J=8.1 \mathrm{~Hz}, 2 \mathrm{H}), 5.95(\mathrm{~d}, J=18.8 \mathrm{~Hz}, 1 \mathrm{H}), 5.82(\mathrm{~d}, J=18.9 \mathrm{~Hz}, 1 \mathrm{H})$, $4.81(\mathrm{~s}, 1 \mathrm{H}), 2.39(\mathrm{~s}, 3 \mathrm{H}), 1.31(\mathrm{~s}, 6 \mathrm{H}), 0.22(\mathrm{~s}, 6 \mathrm{H})$.

${ }^{13} \mathrm{C}$ NMR $\left(101 \mathrm{MHz}, \mathrm{CDCl}_{3}\right) \delta 152.0,143.0,140.2,138.5,133.9,129.6,129.2,127.9,127.3$, $125.2,58.5,21.6,-2.7$.

$\mathrm{N}$-\{(3E)-4-[dimethyl(phenyl)silyl]-2-methylbut-3-en-2-yl\}-methoxybenzene-1sulfonamide (4c)<smiles>COc1ccc(S(=O)(=O)NC(C)(C)/C=C/Sc2ccccc2)cc1</smiles>

The title compound was prepared according to general procedure $\mathrm{E}$, from 4-methoxy-N-(2methylbut-3-yn-2-yl)benzene-1-sulfonamide $(75 \mathrm{mg}, 0.3 \mathrm{mmol}$ ) and dimethylphenylsilane (65 $\mathrm{mg}, 0.47 \mathrm{mmol})$ using $\mathrm{PtCl}_{2}(0.8 \mathrm{mg}, 3 \mu \mathrm{mol})$ and XantPhos $(1.7 \mathrm{mg}, 3 \mu \mathrm{mol})$ which following conversion to the vinyl silane and column chromatography (4:1 Hexane/EtOAc) afforded 4c (115 $\mathrm{mg}, 77 \%)$ as a yellow oil.

$\mathrm{R}_{\mathrm{f}}(4: 1$ Hexane/EtOAc): 0.22

HRMS (ESI) m/z: [M + H] + Calcd for $\mathrm{C}_{20} \mathrm{H}_{27} \mathrm{NO}_{3} \mathrm{SSi} 390.1559$; Found 390.1554

IR umax $\left(\mathrm{cm}^{-1}\right)$ 3292, 3029, 2995, 2951, 1723, 1694

${ }^{1} \mathrm{H}$ NMR $\left(400 \mathrm{MHz}, \mathrm{CDCl}_{3}\right) \delta 7.76(\mathrm{~d}, J=8.6 \mathrm{~Hz}, 2 \mathrm{H}), 7.48-7.40(\mathrm{~m}, 2 \mathrm{H}), 7.37-7.32(\mathrm{~m}$, $3 \mathrm{H}), 6.86(\mathrm{~d}, J=8.8 \mathrm{~Hz}, 2 \mathrm{H}), 5.95(\mathrm{~d}, J=18.8 \mathrm{~Hz}, 1 \mathrm{H}), 5.81(\mathrm{~d}, J=18.9 \mathrm{~Hz}, 1 \mathrm{H}), 4.84(\mathrm{~s}, 1 \mathrm{H})$, $3.82(\mathrm{~s}, 3 \mathrm{H}), 1.30(\mathrm{~s}, 6 \mathrm{H}), 0.23(\mathrm{~s}, 6 \mathrm{H})$.

${ }^{13} \mathrm{C} \mathrm{NMR}\left(101 \mathrm{MHz}, \mathrm{CDCl}_{3}\right) \delta 162.6,152.1,138.5,134.7,133.9,129.4,129.2,127.9,125.1$, $114.1,58.4,55.7,28.0,-2.7$.

\section{$N-\{(3 E)-4-[d i m e t h y l(p h e n y l)$ silyl]-2-methylbut-3-en-2-yl\}-2-chlorobenzene-1-} sulfonamide (4d)<smiles>CC(C)(/C=C/[As]c1ccccc1)NS(=O)(=O)c1ccccc1Cl</smiles>

The title compound was prepared according to general procedure $\mathrm{E}$, from 2-chloro- $\mathrm{N}-(2-$ methylbut-3-yn-2-yl)benzene-1-sulfonamide $(75 \mathrm{mg}, 0.3 \mathrm{mmol})$ and dimethylphenylsilane (60 $\mathrm{mg}, 0.45 \mathrm{mmol})$ using $\mathrm{PtCl}_{2}(0.8 \mathrm{mg}, 3 \mu \mathrm{mol})$ and XantPhos $(1.7 \mathrm{mg}, 3 \mu \mathrm{mol})$ which following conversion to the vinyl silane and column chromatography (4:1 Hexane/EtOAc) afforded $4 \mathbf{d}$ (90 $\mathrm{mg}, 78 \%$ ) as a white solid.

$\mathrm{R}_{\mathrm{f}}(4: 1$ Hexane/EtOAc): 0.29 
HRMS (ESI) m/z: [M + H] + Calcd for $\mathrm{C}_{19} \mathrm{H}_{24} \mathrm{CINO}_{2} \mathrm{SSi} 394.1064$; Found 394.1074

IR umax $\left(\mathrm{cm}^{-1}\right)$ 3292, 3093, 3066, 2976, 2931

${ }^{1} \mathrm{H} \mathrm{NMR}\left(400 \mathrm{MHz}, \mathrm{CDCl}_{3}\right) \delta 8.00$ (dd, $\left.J=7.9,1.5 \mathrm{~Hz}, 1 \mathrm{H}\right), 7.45$ (dd, $\left.J=6.3,4.8 \mathrm{~Hz}, 1 \mathrm{H}\right), 7.43$ $-7.37(\mathrm{~m}, 1 \mathrm{H}), 7.36-7.31(\mathrm{~m}, 2 \mathrm{H}), 7.28(\mathrm{dd}, J=10.8,4.3 \mathrm{~Hz}, 4 \mathrm{H}), 5.91(\mathrm{~d}, J=18.8 \mathrm{~Hz}, 1 \mathrm{H})$, $5.83(\mathrm{~d}, J=18.8 \mathrm{~Hz}, 1 \mathrm{H}), 5.07(\mathrm{~s}, 1 \mathrm{H}), 1.32(\mathrm{~s}, 6 \mathrm{H}), 0.18(\mathrm{~s}, 6 \mathrm{H})$.

${ }^{13} \mathrm{C}$ NMR $\left(101 \mathrm{MHz}, \mathrm{CDCl}_{3}\right) \delta 151.1,140.3,138.3,133.8,133.3,131.5,131.3,130.8,129.2$, $127.8,127.3,125.7,58.7,27.9,-2.8$.

\section{$N-\{(3 E)-4-[$ dimethyl(phenyl)silyl]-2-methylbut-3-en-2-yl\}-3-chlorobenzene-1- sulfonamide (4e)}<smiles>C[SH2+](/C=C/C(C)(C)NS(=O)(=O)c1cccc(Cl)c1)c1ccccc1</smiles>

The title compound was prepared according to general procedure $\mathrm{E}$, from 3-chloro- $\mathrm{N}-(2-$ methylbut-3-yn-2-yl)benzene-1-sulfonamide $(50 \mathrm{mg}, 0.19 \mathrm{mmol})$ and dimethylphenylsilane (40 mg, $0.30 \mathrm{mmol})$ using $\mathrm{PtCl}_{2}(0.5 \mathrm{mg}, 1.9 \mu \mathrm{mol})$ and XantPhos $(1.1 \mathrm{mg}, 1.9 \mu \mathrm{mol})$ which following conversion to the vinyl silane and column chromatography (9:1 Hexane/EtOAc) afforded $4 \mathrm{e}(62 \mathrm{mg}, 81 \%)$ as a pale yellow solid.

$\mathrm{R}_{\mathrm{f}}(4: 1 \mathrm{Hexane} / \mathrm{EtOAc}): 0.31$

HRMS (ESI) m/z: [M + H]+ Calcd for $\mathrm{C}_{19} \mathrm{H}_{24} \mathrm{CINO}_{2} \mathrm{SSi} 394.1064$; Found 394.1073

IR vmax $\left(\mathrm{cm}^{-1}\right)$ 3250, 3069, 3048, 2987, 2866, 1612

${ }^{1} \mathrm{H}$ NMR $\left(400 \mathrm{MHz}, \mathrm{CDCl}_{3}\right) \delta 7.83(\mathrm{t}, J=1.9 \mathrm{~Hz}, 1 \mathrm{H}), 7.70$ (ddd, $\left.J=7.8,1.7,1.0 \mathrm{~Hz}, 1 \mathrm{H}\right), 7.47$ (ddd, $J=8.2,2.1,1.2 \mathrm{~Hz}, 1 \mathrm{H}), 7.45-7.40(\mathrm{~m}, 2 \mathrm{H}), 7.37-7.31(\mathrm{~m}, 4 \mathrm{H}), 5.94(\mathrm{~d}, J=18.9 \mathrm{~Hz}$, $1 \mathrm{H}), 5.84(\mathrm{~d}, \mathrm{~J}=18.8 \mathrm{~Hz}, 1 \mathrm{H}), 4.59(\mathrm{~s}, 1 \mathrm{H}), 1.33(\mathrm{~s}, 6 \mathrm{H}), 0.24(\mathrm{~s}, 6 \mathrm{H})$.

${ }^{13} \mathrm{C} \mathrm{NMR}\left(101 \mathrm{MHz}, \mathrm{CDCl}_{3}\right) \delta 151.4,144.8,138.2,135.1,133.8,132.5,130.3,129.2,127.9$, $127.3,125.9,125.3,58.9,28.1,-2.8$. 


\section{N-\{(3E)-4-[dimethyl(phenyl)silyl]-2-methylbut-3-en-2-yl\}-2-methoxy-5-}

\section{chlorobenzene-1-sulfonamide (4f)}<smiles>COc1ccc(Cl)cc1S(=O)(=O)NC(C)(C)/C=C/Sc1ccccc1</smiles>

The title compound was prepared according to general procedure E, from 2-methoxy-5-chloro$\mathrm{N}$-(2-methylbut-3-yn-2-yl)benzene-1-sulfonamide $\quad(50 \quad \mathrm{mg}, \quad 0.17 \quad \mathrm{mmol})$ and dimethylphenylsilane $(35 \mathrm{mg}, 0.26 \mathrm{mmol})$ using $\mathrm{PtCl}_{2}(0.5 \mathrm{mg}, 1.7 \mu \mathrm{mol})$ and XantPhos $(1.1$ $\mathrm{mg}, 1.7 \mu \mathrm{mol})$ which following conversion to the vinyl silane and column chromatography (9:1 Hexane/EtOAc) afforded $\mathbf{4 f}(61 \mathrm{mg}, 83 \%)$ as a white solid.

$\mathrm{R}_{\mathrm{f}}(4: 1$ Hexane/EtOAc): 0.21

HRMS (ESI) m/z: [M + H]+ Calcd for $\mathrm{C}_{20} \mathrm{H}_{26} \mathrm{CINO}_{3} \mathrm{SSi}$ 424.1169; Found 424.1157

IR umax $\left(\mathrm{cm}^{-1}\right)$ 3250, 3070, 3049, 2972, 1611

${ }^{1} \mathrm{H}$ NMR $\left(400 \mathrm{MHz}, \mathrm{CDCl}_{3}\right) \delta 7.83(\mathrm{~d}, J=2.7 \mathrm{~Hz}, 1 \mathrm{H}), 7.44-7.37(\mathrm{~m}, 3 \mathrm{H}), 7.36-7.31(\mathrm{~m}$, $3 \mathrm{H}), 6.87(\mathrm{~d}, J=8.9 \mathrm{~Hz}, 1 \mathrm{H}), 5.89(\mathrm{~d}, J=18.8 \mathrm{~Hz}, 1 \mathrm{H}), 5.80(\mathrm{~d}, J=18.9 \mathrm{~Hz}, 1 \mathrm{H}), 5.00(\mathrm{~s}, 1 \mathrm{H})$, $3.88(\mathrm{~s}, 3 \mathrm{H}), 1.31(\mathrm{~s}, 6 \mathrm{H}), 0.20(\mathrm{~s}, 6 \mathrm{H})$.

${ }^{13} \mathrm{C}$ NMR $\left(101 \mathrm{MHz}, \mathrm{CDCl}_{3}\right) \delta$ 154.5, 151.3, 138.2, 133.9, 133.7, 133.1, 132.3, 129.2, 127.9, $126.1,125.5,113.5,58.5,56.6,27.9,-2.8$.

\section{$\underline{\mathrm{N}}\{(3 \mathrm{E})-4-[$ dimethyl(phenyl)silyl]-2-methylbut-3-en-2-yl\}acetamide (4g)}<smiles>CC(=O)NC(C)(C)/C=C/Sc1ccccc1</smiles>

The title compound was prepared according to general procedure $\mathrm{E}$, from $\mathrm{N}$-(2-methylbut-3yn-2-yl)acetamide (50 mg, $0.4 \mathrm{mmol}$ ) and dimethylphenylsilane ( $82 \mathrm{mg}, 0.6 \mathrm{mmol})$ using $\mathrm{PtCl}_{2}$ $(1.1 \mathrm{mg}, 4 \mu \mathrm{mol})$ and XantPhos $(2.3 \mathrm{mg}, 4 \mu \mathrm{mol})$ which following conversion to the vinyl silane and column chromatography (4:1 Hexane/EtOAc) afforded $\mathbf{4 g}(87 \mathrm{mg}, 83 \%)$ as a white solid.

$\mathrm{R}_{\mathrm{f}}(4: 1$ Hexane/EtOAc): 0.33

IR umax $\left(\mathrm{cm}^{-1}\right) 3278,3069,3012,2963,1643$

HRMS (ESI) m/z: [M + H] + Calcd for $\mathrm{C}_{15} \mathrm{H}_{24} \mathrm{NOSi} 262.1627$; Found 262.1622

${ }^{1} \mathrm{H}$ NMR (400 MHz, $\left.\mathrm{CDCl}_{3}\right) \delta 7.50(\mathrm{~s}, 2 \mathrm{H}), 7.39-7.32(\mathrm{~m}, 3 \mathrm{H}), 6.23(\mathrm{~d}, J=19.0 \mathrm{~Hz}, 1 \mathrm{H}), 5.80$ $(\mathrm{d}, J=19.0 \mathrm{~Hz}, 1 \mathrm{H}), 5.41-5.30(\mathrm{~m}, 1 \mathrm{H}), 1.94(\mathrm{~s}, 3 \mathrm{H}), 1.43(\mathrm{~s}, 6 \mathrm{H}), 0.34(\mathrm{~s}, 6 \mathrm{H})$.

${ }^{13} \mathrm{C}$ NMR $\left(101 \mathrm{MHz}, \mathrm{CDCl}_{3}\right) \delta 169.3,153.2,138.9,134.0,129.1,127.9,123.4,55.8,27.2$, 24.7, -2.4 . 


\section{2-chloro- $\mathrm{N}-\{(3 E)-4-[$ dimethyl(phenyl) silyl]-2-methylbut-3-en-2-yl\}benzamide (4h)}<smiles>CC(C)(/C=C/Sc1ccccc1)NC(=O)c1ccccc1Cl</smiles>

The title compound was prepared according to general procedure $\mathrm{E}$, from 2-chloro- $\mathrm{N}$-(2methylbut-3-yn-2-yl)benzamide $(75 \mathrm{mg}, 0.33 \mathrm{mmol}$ ) and dimethylphenylsilane (73 $\mathrm{mg}, 0.54$ mmol) using PtCl2 $(1.8 \mathrm{mg}, 6.7 \mu \mathrm{mol})$ and XantPhos $(3.9 \mathrm{mg}, 6.7 \mu \mathrm{mol})$ which following conversion to the vinyl silane and column chromatography (4:1 Hexane/EtOAc) afforded $\mathbf{4 h}$ (109 mg, $90 \%$ ) as an orange oil.

$\mathrm{R}_{\mathrm{f}}(4: 1$ Hexane/EtOAc): 0.43

IR umax $\left(\mathrm{cm}^{-1}\right)$ 3429, 3281, 3068, 2961, 1649

HRMS (ESI) m/z: [M + H] + Calcd for $\mathrm{C}_{20} \mathrm{H}_{24}$ CINOSi 358.1394; Found 358.1388

${ }^{1} \mathrm{H}$ NMR (400 MHz, $\left.\mathrm{CDCl}_{3}\right) \delta 7.60(\mathrm{dd}, J=6.0,1.5 \mathrm{~Hz}, 1 \mathrm{H}), 7.54(\mathrm{dt}, J=7.8,3.2 \mathrm{~Hz}, 2 \mathrm{H}), 7.39$ $-7.35(\mathrm{~m}, 4 \mathrm{H}), 7.35-7.29(\mathrm{~m}, 2 \mathrm{H}), 6.34(\mathrm{~d}, J=19.0 \mathrm{~Hz}, 1 \mathrm{H}), 6.07(\mathrm{~s}, 1 \mathrm{H}), 5.95(\mathrm{~d}, J=18.9$ $\mathrm{Hz}, 1 \mathrm{H}), 1.57(\mathrm{~s}, 6 \mathrm{H}), 0.37(\mathrm{~s}, 6 \mathrm{H})$.

${ }^{13} \mathrm{C}$ NMR $\left(101 \mathrm{MHz}, \mathrm{CDCl}_{3}\right) \delta 165.7,152.7,138.8,136.3,134.0,133.2,131.0,130.2,129.9$, $129.1,127.9,127.2,124.0,77.5,77.2,76.8,56.7,27.2,-2.4$.

\section{$\mathrm{N}$-\{(3E)-4-[dimethyl(phenyl)silyl]-2-methylbut-3-en-2-yl\}furan-2-carboxamide}

(4i)<smiles>CC(C)(/C=C/[AsH2]c1ccccc1)NC(=O)c1ccco1</smiles>

The title compound was prepared according to general procedure $\mathrm{E}$, from $\mathrm{N}$-(2-methylbut-3yn-2-yl)furan-2-carboxamide $(50 \mathrm{mg}, 0.28 \mathrm{mmol}$ ) and dimethylphenylsilane $(61 \mathrm{mg}, 0.42$ mmol) using $\mathrm{PtCl}_{2}(1.5 \mathrm{mg}, 5.7 \mu \mathrm{mol})$ and XantPhos $(3.3 \mathrm{mg}, 5.7 \mu \mathrm{mol})$ which following conversion to the vinyl silane and column chromatography (4:1 Hexane/EtOAc) afforded $\mathbf{4 i}$ (89 $\mathrm{mg}, 84 \%$ ) as a white solid.

$\mathrm{R}_{\mathrm{f}}(4: 1$ Hexane/EtOAc): 0.38

IR umax $\left(\mathrm{cm}^{-1}\right)$ 3243, 3120, 3063, 2972, 1640, 1572

HRMS (ESI) m/z: [M + H]+ Calcd for $\mathrm{C}_{18} \mathrm{H}_{23} \mathrm{NO}_{2} \mathrm{Si} 314.1576$; Found 314.1571

${ }^{1} \mathrm{H}$ NMR $\left(400 \mathrm{MHz}, \mathrm{CDCl}_{3}\right) \delta 7.53(\mathrm{dd}, J=6.2,3.3 \mathrm{~Hz}, 2 \mathrm{H}), 7.41(\mathrm{~s}, 1 \mathrm{H}), 7.38-7.32(\mathrm{~m}$, $3 \mathrm{H}), 7.07(\mathrm{~d}, J=3.0 \mathrm{~Hz}, 1 \mathrm{H}), 6.48(\mathrm{dd}, J=3.5,1.8 \mathrm{~Hz}, 1 \mathrm{H}), 6.31(\mathrm{~m}, 2 \mathrm{H}), 5.88(\mathrm{~d}, J=19.0$ $\mathrm{Hz}, 1 \mathrm{H}), 1.54(\mathrm{~s}, 6 \mathrm{H}), 0.35(\mathrm{~s}, 6 \mathrm{H})$. 
${ }^{13} \mathrm{C} \mathrm{NMR}\left(101 \mathrm{MHz}, \mathrm{CDCl}_{3}\right) \delta 157.6,152.8,148.8,143.5,138.8,134.0,133.1,129.1,127.9$, $123.7,113.8,112.23,55.92,27.31,-2.41$.

\section{$\mathrm{N}$-\{(3E)-4-[dimethyl(phenyl)silyl]-2-methylbut-3-en-2-yl\}thiophene-2-}

\section{carboxamide (4i)}<smiles>CC(C)(C=Cc1ccccc1)NC(=O)c1cccs1</smiles>

The title compound was prepared according to general procedure $\mathrm{E}$, from $\mathrm{N}$-(2-methylbut-3yn-2-yl)thiophene-2-carboxamide $(50 \mathrm{mg}, 0.28 \mathrm{mmol})$ and dimethylphenylsilane $(61 \mathrm{mg}, 0.42$ mmol) using $\mathrm{PtCl}_{2}(0.7 \mathrm{mg}, 2.8 \mu \mathrm{mol})$ and XantPhos $(1.7 \mathrm{mg}, 2.8 \mu \mathrm{mol})$ which following conversion to the vinyl silane and column chromatography (4:1 Hexane/EtOAc) afforded $\mathbf{4 j}$ (76 $\mathrm{mg}, 87 \%$ ) as a white solid.

$\mathrm{R}_{\mathrm{f}}(4: 1$ Hexane/EtOAc): 0.42

IR umax $\left(\mathrm{cm}^{-1}\right) 3255,3103,3089,2966,1622$

HRMS (ESI) m/z: [M + H]+ Calcd for $\mathrm{C}_{18} \mathrm{H}_{23}$ NOSSi 330.1348; Found 330.1356

${ }^{1} \mathrm{H}$ NMR $\left(400 \mathrm{MHz}, \mathrm{CDCl}_{3}\right) \delta 7.53$ (dd, $\left.J=6.3,3.1 \mathrm{~Hz}, 2 \mathrm{H}\right), 7.48-7.40(\mathrm{~m}, 2 \mathrm{H}), 7.39-7.32$ $(\mathrm{m}, 3 \mathrm{H}), 7.09-7.02(\mathrm{~m}, 1 \mathrm{H}), 6.33(\mathrm{~d}, J=18.9 \mathrm{~Hz}, 1 \mathrm{H}), 5.90(\mathrm{~s}, 1 \mathrm{H}) 5.89(\mathrm{~d}, J=18.9 \mathrm{~Hz}, 1 \mathrm{H})$, $1.55(\mathrm{~s}, 6 \mathrm{H}), 0.36(\mathrm{~s}, 6 \mathrm{H})$.

${ }^{13} \mathrm{C}$ NMR $\left(101 \mathrm{MHz} . \mathrm{CDCl}_{3}\right) \delta 161.0,152.9,140.4,138.8,134.0,129.6,129.1,127.9,127.7$, 123.8, 56.4, 27.3.

\section{tert-butyl $\{(3 E)-4-[$ dimethyl(phenyl)silyl]-2-methylbut-3-en-2-yl\}carbamate (4k)}<smiles>CC(C)(/C=C/Sc1ccccc1)NC(=O)OC(C)(C)C</smiles>

The title compound was prepared according to general procedure $\mathrm{E}$, from tert-butyl (2methylbut-3-yn-2-yl)carbamate $(50 \mathrm{mg}, 0.27 \mathrm{mmol})$ and dimethylphenylsilane $(69 \mathrm{mg}, 0.51$ mmol) using $\mathrm{PtCl}_{2}(0.7 \mathrm{mg}, 2.7 \mu \mathrm{mol})$ and XantPhos $(1.7 \mathrm{mg}, 2.7 \mu \mathrm{mol})$ which following conversion to the vinyl silane and column chromatography (4:1 Hexane/EtOAc) afforded $\mathbf{4 k}$ (74 $\mathrm{mg}, 86 \%$ ) as a pale yellow solid.

$\mathrm{R}_{\mathrm{f}}(4: 1 \mathrm{Hexane} / \mathrm{EtOAc}): 0.41$

HRMS (ESI) m/z: [M + H]+ Calcd for $\mathrm{C}_{18} \mathrm{H}_{29} \mathrm{NO}_{2} \mathrm{Si} 320.2046$; Found 320.2040

IR umax $\left(\mathrm{cm}^{-1}\right)$ 3358, 3069, 2973, 2930, 1723, 1695

${ }^{1} \mathrm{H} \mathrm{NMR}\left(400 \mathrm{MHz}, \mathrm{CDCl}_{3}\right) \delta 7.53-7.47(\mathrm{~m}, J=6.4,3.0 \mathrm{~Hz}, 2 \mathrm{H}), 7.35-7.31(\mathrm{~m}, 3 \mathrm{H}), 6.15$ $(\mathrm{d}, J=18.9 \mathrm{~Hz}, 1 \mathrm{H}), 5.80(\mathrm{~d}, J=18.9 \mathrm{~Hz}, 1 \mathrm{H}), 4.58(\mathrm{~s}, 1 \mathrm{H}), 1.41(\mathrm{~s}, 9 \mathrm{H}), 1.36(\mathrm{~s}, 6 \mathrm{H}), 0.33(\mathrm{~s}$, $6 \mathrm{H})$. 
${ }^{13} \mathrm{C}$ NMR $\left(101 \mathrm{MHz}, \mathrm{CDCl}_{3}\right) \delta 153.7,139.0,134.2,134.0,129.1,127.9,123.1,54.8,28.5$, 27.6, -2.3.

(4b,8a,9,9a-tetrahydro-4aH-fluoren-9-yl)methyl \{(3E)-4-[dimethyl(phenyl)silyl]-2methylbut-3-en-2-yl\}carbamate (4I)

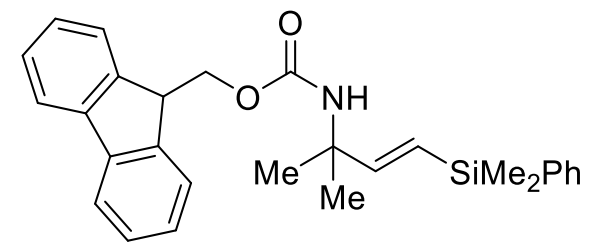

The title compound was prepared according to general procedure $E$, from $(4 b, 8 a, 9,9 a-$ tetrahydro-4aH-fluoren-9-yl)methyl (2-methylbut-3-yn-2-yl)carbamate $(75 \mathrm{mg}, 0.31 \mathrm{mmol})$ and dimethylphenylsilane $(69 \mathrm{mg}, 0.51 \mathrm{mmol})$ using $\mathrm{PtCl}_{2}(0.9 \mathrm{mg}, 3.1 \mu \mathrm{mol})$ and XantPhos $(1.8$ $\mathrm{mg}, 3.2 \mu \mathrm{mol}$ ) which following conversion to the vinyl silane and column chromatography (4:1 Hexane/EtOAc) afforded 4 I (92 mg, $78 \%$ ) as a colourless oil..

$\mathrm{R}_{\mathrm{f}}(4: 1$ Hexane/EtOAc): 0.47

HRMS (ESI) m/z: [M + H] + Calcd for $\mathrm{C}_{28} \mathrm{H}_{31} \mathrm{NO}_{2} \mathrm{Si} 442.2202$; Found 442.2197

IR umax $\left(\mathrm{cm}^{-1}\right)$ 3412, 3340, 3067, 2961, 1708

${ }^{1} \mathrm{H}$ NMR $\left(400 \mathrm{MHz}, \mathrm{CDCl}_{3}\right) \delta 7.76(\mathrm{~d}, J=7.5 \mathrm{~Hz}, 2 \mathrm{H}), 7.59(\mathrm{~d}, J=7.4 \mathrm{~Hz}, 2 \mathrm{H}), 7.50(\mathrm{~s}, 2 \mathrm{H})$, $7.39(\mathrm{t}, J=7.4 \mathrm{~Hz}, 2 \mathrm{H}), 7.36-7.25(\mathrm{~m}, J=14.7,7.3 \mathrm{~Hz}, 5 \mathrm{H}), 6.19(\mathrm{~d}, J=18.8 \mathrm{~Hz}, 1 \mathrm{H}), 5.82$ $(\mathrm{d}, J=18.9 \mathrm{~Hz}, 1 \mathrm{H}), 4.81(\mathrm{~s}, 1 \mathrm{H}), 4.37(\mathrm{~d}, J=5.0 \mathrm{~Hz}, 2 \mathrm{H}), 4.19(\mathrm{t}, J=6.2 \mathrm{~Hz}, 1 \mathrm{H}), 1.41(\mathrm{~s}$, $6 \mathrm{H}), 0.33(\mathrm{~s}, 6 \mathrm{H})$.

${ }^{13} \mathrm{C}$ NMR $\left(101 \mathrm{MHz}, \mathrm{CDCl}_{3}\right) \delta 153.1,144.2,141.4,138.9,134.0,129.1,127.9,127.8,127.2$, $125.2,123.7,120.1,66.1,55.1,47.5,27.4,-2.4$.

\section{Synthesis of N-Sulfonyl Aziridines}




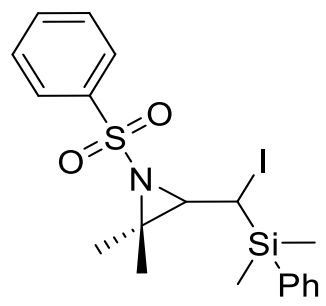

The title compound was prepared according to general procedure $F$, from $N-\{(3 E)-4-$ [dimethyl(phenyl)silyl]-2-methylbut-3-en-2-yl\}-benzene-1-sulfonamide (30 mg, $0.084 \mathrm{mmol}$ ) and $\mathrm{N}$-lodosuccinimide (56 $\mathrm{mg}, 0.25 \mathrm{mmol})$ which following conversion to the aziridine and column chromatography (4:1 Hexane/EtOAc) afforded 5 a as a colourless oil (34mg, $84 \%)$.

$\mathrm{R}_{\mathrm{f}}(4: 1$ Hexane/EtOAc): 0.43

HRMS (ESI) m/z: [M + H] + Calcd for $\mathrm{C}_{19} \mathrm{H}_{25} \mathrm{INO}_{2} \mathrm{SSi} 486.0420$; Found 486.0412

IR umax $\left(\mathrm{cm}^{-1}\right)$ 3070, 2981, 2940, 2922, 1593

${ }^{1} \mathrm{H}$ NMR $\left(400 \mathrm{MHz}, \mathrm{CDCl}_{3}\right) \delta 7.93-7.85(\mathrm{~m}, 2 \mathrm{H}), 7.61-7.54(\mathrm{~m}, 3 \mathrm{H}), 7.48(\mathrm{t}, J=7.6 \mathrm{~Hz}$, $2 \mathrm{H}), 7.41-7.33(\mathrm{~m}, 3 \mathrm{H}), 3.21(\mathrm{~d}, J=11.7 \mathrm{~Hz}, 1 \mathrm{H}), 2.94(\mathrm{~d}, J=11.7 \mathrm{~Hz}, 1 \mathrm{H}), 1.57(\mathrm{~s}, 3 \mathrm{H})$, $1.30(\mathrm{~s}, 3 \mathrm{H}), 0.35(\mathrm{~s}, 3 \mathrm{H}), 0.25(\mathrm{~s}, 3 \mathrm{H})$.

${ }^{13} \mathrm{C}$ NMR $\left(101 \mathrm{MHz}, \mathrm{CDCl}_{3}\right) \delta 140.7,135.5,134.6,133.2,129.9,128.9,127.8,127.7,53.1$, $53.0,29.8,21.0,19.7,15.3,-2.3,-4.3$.

\section{3-\{[dimethyl(phenyl)silyl](iodo)methyl\}-2,2-dimethyl-1-(4-methylbenzene-1-} sulfonyl)aziridine (5b)

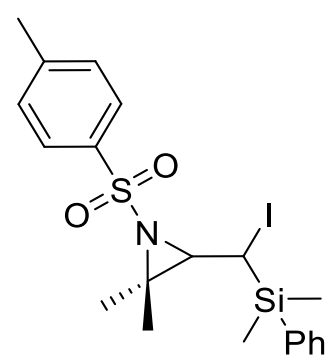

The title compound was prepared according to general procedure $F$, from $N-\{(3 E)-4-$ [dimethyl(phenyl)silyl]-2-methylbut-3-en-2-yl\}-4-methylbenzene-1-sulfonamide (50 mg, 0.134 $\mathrm{mmol}$ ) and $\mathrm{N}$-lodosuccinimide $(90 \mathrm{mg}, 0.040 \mathrm{mmol}$ ) which following conversion to the aziridine and column chromatography (4:1 Hexane/EtOAc) afforded $\mathbf{5 b}$ as a white solid (56 $\mathrm{mg}, 84 \%)$.

$\mathrm{R}_{\mathrm{f}}(4: 1$ Hexane/EtOAc): 0.41

IR umax $\left(\mathrm{cm}^{-1}\right)$ 3069, 2991, 2968, 2922, 1598

HRMS (ESI) m/z: [M + H] + Calcd for $\mathrm{C}_{20} \mathrm{H}_{27} \mathrm{NNO}_{2}$ SSi 500.0576; Found 500.0575 
${ }^{1} \mathrm{H}$ NMR $\left(400 \mathrm{MHz}, \mathrm{CDCl}_{3}\right) \delta 7.76(\mathrm{~d}, J=8.3 \mathrm{~Hz}, 2 \mathrm{H}), 7.57(\mathrm{~d}, J=7.8 \mathrm{~Hz}, 2 \mathrm{H}), 7.38$ (dd, $J=$ 9.8, 7.0 Hz, 3H), $7.27(\mathrm{~s}, 2 \mathrm{H}), 3.19(\mathrm{~d}, J=11.8 \mathrm{~Hz}, 1 \mathrm{H}), 2.94(\mathrm{~d}, J=11.7 \mathrm{~Hz}, 1 \mathrm{H}), 2.41(\mathrm{~s}$, $3 \mathrm{H}), 1.54(\mathrm{~s}, 3 \mathrm{H}), 1.28(\mathrm{~s}, 3 \mathrm{H}), 0.37(\mathrm{~s}, 3 \mathrm{H}), 0.28(\mathrm{~s}, 3 \mathrm{H})$.

${ }^{13} \mathrm{C}$ NMR $\left(101 \mathrm{MHz}, \mathrm{CDCl}_{3}\right) \delta 144.0,137.8,135.5,134.7,129.9,129.5,127.8,127.7,52.9$, $52.7,21.7,20.9,19.7,15.4,-2.3,-4.2$.

\section{3-\{[dimethyl(phenyl)silyl](iodo)methyl\}-1-(4-methoxybenzene-1-sulfonyl)-2,2- dimethylaziridine (5c)}

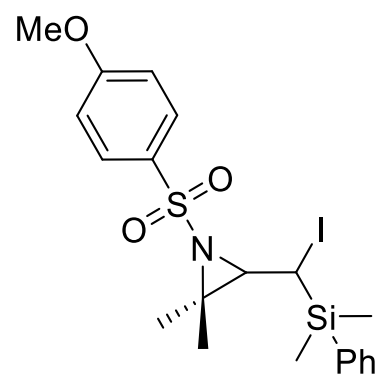

The title compound was prepared according to general procedure $F$, from $N-\{(3 E)-4-$ [dimethyl(phenyl)silyl]-2-methylbut-3-en-2-yl\}-4- benzene-1-sulfonamide (40 mg, $0.103 \mathrm{mmol}$ ) and N-lodosuccinimide (69 $\mathrm{mg}, 0.31 \mathrm{mmol}$ ) which following conversion to the aziridine and column chromatography (4:1 Hexane/EtOAc) afforded $\mathbf{5 c}$ as a colourless oil (45 $\mathrm{mg}, 85 \%)$.

$\mathrm{R}_{\mathrm{f}}(4: 1$ Hexane/EtOAc): 0.32

IR umax $\left(\mathrm{cm}^{-1}\right)$ 3071, 3002, 2967, 2933, 2851

HRMS (ESI) m/z: [M + H] + Calcd for $\mathrm{C}_{20} \mathrm{H}_{27} \mathrm{INO}_{3} \mathrm{SSi}$ 516.0526; Found 516.0526

${ }^{1} \mathrm{H}$ NMR $\left(400 \mathrm{MHz}, \mathrm{CDCl}_{3}\right) \delta 7.86-7.76(\mathrm{~m}, 2 \mathrm{H}), 7.57(\mathrm{dd}, J=7.6,1.6 \mathrm{~Hz}, 2 \mathrm{H}), 7.41-7.32$ $(\mathrm{m}, 3 \mathrm{H}), 6.96-6.88(\mathrm{~m}, 2 \mathrm{H}), 3.86(\mathrm{~s}, 3 \mathrm{H}), 3.18(\mathrm{~d}, J=11.7 \mathrm{~Hz}, 1 \mathrm{H}), 2.94(\mathrm{~d}, J=11.7 \mathrm{~Hz}, 1 \mathrm{H})$, $1.54(\mathrm{~s}, 3 \mathrm{H}), 1.27(\mathrm{~s}, 3 \mathrm{H}), 0.38(\mathrm{~s}, 3 \mathrm{H}), 0.29(\mathrm{~s}, 3 \mathrm{H})$.

${ }^{13} \mathrm{C}$ NMR $\left(101 \mathrm{MHz}, \mathrm{CDCl}_{3}\right) \delta 163.27,134.64,134.54,132.38,129.88,129.69,127.82$, $114.04,55.72,52.88,52.48,20.79,19.77,15.43,-2.33,-4.12$.

\section{3-\{[dimethyl(phenyl)silyl](iodo)methyl\}-1-(2-chlorobenzene-1-sulfonyl)-2,2- dimethylaziridine (5d)}

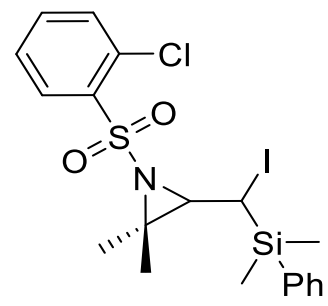

The title compound was prepared according to general procedure $F$, from $N-\{(3 E)-4-$ [dimethyl(phenyl)silyl]-2-methylbut-3-en-2-yl\}-2-chlorobenzene-1-sulfonamide (30 mg, 0.075 
$\mathrm{mmol}$ ) and $\mathrm{N}$-lodosuccinimide (52 $\mathrm{mg}, 0.225 \mathrm{mmol}$ ) which following conversion to the aziridine and column chromatography (4:1 Hexane/EtOAc) afforded $\mathbf{5 d}$ as a white solid.

$\mathrm{R}_{\mathrm{f}}(4: 1$ Hexane/EtOAc): 0.47

HRMS (ESI) m/z: [M + H]+ Calcd for $\mathrm{C}_{19} \mathrm{H}_{24} \mathrm{ClINO}_{2} \mathrm{SSi}$ 520.0030; Found 520.0011

IR umax $\left(\mathrm{cm}^{-1}\right)$ 3068, 2971, 2967, 1563

${ }^{1} \mathrm{H}$ NMR $\left(400 \mathrm{MHz}, \mathrm{CDCl}_{3}\right) \delta 8.05-7.95(\mathrm{~m}, 1 \mathrm{H}), 7.55-7.45(\mathrm{~m}, 4 \mathrm{H}), 7.39-7.30(\mathrm{~m}, 4 \mathrm{H})$, $3.30(\mathrm{~d}, J=11.7 \mathrm{~Hz}, 1 \mathrm{H}), 2.91(\mathrm{~d}, J=11.7 \mathrm{~Hz}, 1 \mathrm{H}), 1.69(\mathrm{~s}, 3 \mathrm{H}), 1.40(\mathrm{~s}, 3 \mathrm{H}), 0.28(\mathrm{~s}, 3 \mathrm{H})$, $0.21(\mathrm{~s}, 3 \mathrm{H})$.

${ }^{13} \mathrm{C}$ NMR $\left(101 \mathrm{MHz}, \mathrm{CDCl}_{3}\right) \delta 138.6,135.4,134.5,134.0,133.3,132.1,130.1,129.9,127.8$, $126.7,54.7,53.4,22.6,19.4,15.2,-2.8,-4.2$.

\section{3-\{[dimethyl(phenyl)silyl](iodo)methyl\}-1-(3-chlorobenzene-1-sulfonyl)-2,2-}

\section{dimethylaziridine (5e)}

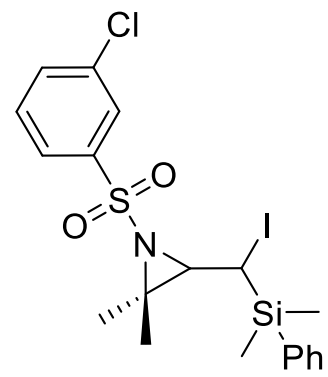

The title compound was prepared according to general procedure $F$, from $N-\{(3 E)-4-$ [dimethyl(phenyl)silyl]-2-methylbut-3-en-2-yl\}-3-chlorobenzene-1-sulfonamide (30 mg, 0.075 $\mathrm{mmol}$ ) and $\mathrm{N}$-lodosuccinimide ( $52 \mathrm{mg}, 0.225 \mathrm{mmol}$ ) which following conversion to the aziridine and column chromatography (4:1 Hexane/EtOAc) afforded $5 \mathrm{e}$ as a white solid (31 $\mathrm{mg}, 78 \%)$.

$R_{f}(4: 1$ Hexane/EtOAc): 0.48

HRMS (ESI) m/z: [M + H] + Calcd for $\mathrm{C}_{19} \mathrm{H}_{24} \mathrm{CIINO}_{2} \mathrm{SSi}$ 520.0030; Found 520.0016

IR umax $\left(\mathrm{cm}^{-1}\right)$ 3043, 2964, 2911, 2879, 1589

${ }^{1} \mathrm{H}$ NMR $\left(400 \mathrm{MHz}, \mathrm{CDCl}_{3}\right) \delta 7.85(\mathrm{t}, J=1.8 \mathrm{~Hz}, 1 \mathrm{H}), 7.74(\mathrm{dd}, J=6.7,1.2 \mathrm{~Hz}, 1 \mathrm{H}), 7.56-$ $7.49(\mathrm{~m}, 3 \mathrm{H}), 7.43-7.33(\mathrm{~m}, 4 \mathrm{H}), 3.24(\mathrm{~d}, J=11.7 \mathrm{~Hz}, 1 \mathrm{H}), 2.95(\mathrm{~d}, J=11.8 \mathrm{~Hz}, 1 \mathrm{H}), 1.60$ $(\mathrm{s}, 3 \mathrm{H}), 1.33(\mathrm{~s}, 3 \mathrm{H}), 0.33(\mathrm{~s}, 3 \mathrm{H}), 0.29(\mathrm{~s}, 3 \mathrm{H})$.

${ }^{13} \mathrm{C} \mathrm{NMR}\left(101 \mathrm{MHz}, \mathrm{CDCl}_{3}\right) \delta 142.4,135.1,134.5,133.2,130.2,130.0,127.9,127.8,125.7$, 53.6, 29.8, 21.4, 19.7, 14.8, -2.5, -3.8. 


\section{3-\{[dimethyl(phenyl)silyl](iodo)methyl\}-1-(2-methoxy-5-chlorobenzene-1-}

\section{sulfonyl)-2,2-dimethylaziridine (5f)}<smiles>COc1ccc(Cl)cc1S(=O)(=O)N1C(C(I)[Si](C)(C)c2ccccc2)C1(C)C</smiles>

The title compound was prepared according to general procedure $F$, from $N-\{(3 E)-4-$ [dimethyl(phenyl)silyl]-2-methylbut-3-en-2-yl\}-2-methoxy-5-chlorobenzene-1-sulfonamide (30 $\mathrm{mg}, 0.070 \mathrm{mmol}$ ) and $\mathrm{N}$-lodosuccinimide $(48 \mathrm{mg}, 0.210 \mathrm{mmol}$ ) which following conversion to the aziridine and column chromatography (4:1 Hexane/EtOAc) afforded $\mathbf{5 f}$ as a yellow oil (35 $\mathrm{mg}, 90 \%)$.

$\mathrm{R}_{\mathrm{f}}(4: 1$ Hexane/EtOAc): 0.33

HRMS (ESI) m/z: [M + H]+ Calcd for $\mathrm{C}_{20} \mathrm{H}_{26} \mathrm{CIINO}_{3} \mathrm{SSi}$ 550.0136; Found 550.0126

IR umax $\left(\mathrm{cm}^{-1}\right)$ 3082, 2950, 2909, 28921578

${ }^{1} \mathrm{H}$ NMR $\left(400 \mathrm{MHz}, \mathrm{CDCl}_{3}\right) \delta 7.77(\mathrm{~d}, J=2.7 \mathrm{~Hz}, 1 \mathrm{H}), 7.56$ (dd, $\left.J=7.7,1.7 \mathrm{~Hz}, 2 \mathrm{H}\right), 7.46$ (dd, $J=8.8,2.7 \mathrm{~Hz}, 1 \mathrm{H}), 7.39-7.31(\mathrm{~m}, 3 \mathrm{H}), 6.93(\mathrm{~d}, J=8.9 \mathrm{~Hz}, 1 \mathrm{H}), 3.96(\mathrm{~s}, 3 \mathrm{H}), 3.27(\mathrm{~d}, J=$ $11.7 \mathrm{~Hz}, 1 \mathrm{H}), 2.94(\mathrm{~d}, J=11.6 \mathrm{~Hz}, 1 \mathrm{H}), 1.66(\mathrm{~s}, 3 \mathrm{H}), 1.36(\mathrm{~s}, 3 \mathrm{H}), 0.37(\mathrm{~d}, J=14.9 \mathrm{~Hz}, 6 \mathrm{H})$.

${ }^{13} \mathrm{C}$ NMR $\left(101 \mathrm{MHz}, \mathrm{CDCl}_{3}\right) \delta 155.9,135.5,134.5,134.3,130.3,129.8,129.3,127.8,125.2$, $113.9,56.7,54.8,52.8,22.3,19.7,15.7,-2.9,-3.8$.

3-\{[dimethyl(phenyl)silyl](bromo)methyl\}-1-(2-chlorobenzene-1-sulfonyl)-2,2dimethylaziridine $(5 \mathrm{~g})$

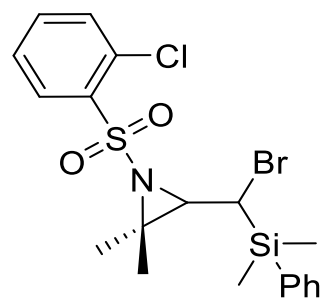

The title compound was prepared according to general procedure $F$, from $N-\{(3 E)-4-$ [dimethyl(phenyl)silyl]-2-methylbut-3-en-2-yl\}-2-chlorobenzene-1-sulfonamide (30 mg, 0.075 $\mathrm{mmol}$ ) and N-Bromosuccinimide $(41 \mathrm{mg}, 0.225 \mathrm{mmol})$ which following conversion to the aziridine and column chromatography (4:1 Hexane/EtOAc) afforded 56 as a colourless oil (25mg, 68\%).

IR umax $\left(\mathrm{cm}^{-1}\right)$ 3056, 3033, 2978, 28981584

HRMS (ESI) m/z: [M + H]+ Calcd for $\mathrm{C}_{19} \mathrm{H}_{24} \mathrm{ClBrNO}_{2} \mathrm{SSi} 472.0169$; Found 472.0173 
${ }^{1} \mathrm{H} \mathrm{NMR}\left(400 \mathrm{MHz} \mathrm{CDCl}_{3}\right) \delta 8.00$ (dd, $\left.J=7.9,1.4 \mathrm{~Hz}, 1 \mathrm{H}\right), 7.56-7.49(\mathrm{~m}, 4 \mathrm{H}), 7.37$ (ddd, $J$ $=9.8,4.4,2.7 \mathrm{~Hz}, 4 \mathrm{H}), 3.24(\mathrm{~d}, J=11.4 \mathrm{~Hz}, 1 \mathrm{H}), 3.01(\mathrm{~d}, J=11.4 \mathrm{~Hz}, 1 \mathrm{H}), 1.72(\mathrm{~s}, 3 \mathrm{H})$, $1.45(\mathrm{~s}, 3 \mathrm{H}), 0.26(\mathrm{~s}, 3 \mathrm{H}), 0.22(\mathrm{~s}, 3 \mathrm{H})$.

${ }^{13} \mathrm{C} \mathrm{NMR}\left(101 \mathrm{MHz}, \mathrm{CDCl}_{3}\right) \delta 138.62,134.56,133.99,133.32,133.07,132.12,130.13$, $129.92,127.85,126.72,54.12,51.91,37.82,22.87,19.97,-3.72,-4.83$. 


\section{References}

1. N. Sakai, H. Hori and Y. Ogiwara, Eur. J. Org. Chem., 2015, 2015, 1905-1909.

2. Y. Imada, M. Yuasa, I. Nakamura and S. Murahashi, J. Org. Chem., 1994, 59, $2282-$ 2284.

3. S. Hayashi, H. Yorimitsu and K. Oshima, Angew. Chem. Int. Ed., 2009, 48, 72247226.

4. E. Y. Schmidt,, I. A. Bidusenko,, N. I. Protsuk,, Y. V. Demyanov,, I. A. Ushakov and B. A. Trofimov, Eur. J. Org. Chem., 2019, 5875-5881

5. R. Liu, Y. Chou, B. Lian. D, Fang, M. Gao, T. Cheng and G. Liu , Org. Lett. 2019, 21, $6815-6820$

6. S. Yasuhara, M. Sasa, T. Kusakabe, H. Takayama, M. Kimura, T. Mochida, K. Kato, Angew. Chem. Int. Ed., 2011, 50, 3912- 3915

7. Y. Zhu, P. Liu, D. Wang, J. Zhang, J. Cheng, Y. Ma, X. Zou and H. Yang, Chin. J. Chem., 2013, 31, 173-181

8. Fukumoto, Y.; Tamura, Y.; Iyori, Y.; Chatani, N. J. Org. Chem. 2016, 81, 3161- 3167

9. K. B. Salah, S. Das, N. Ruiz, V. Andreu, J. Martinez, E. Wenger, M. Amblard, C. Didierjean , B. Legrand and N. Inguimbert , Org. Biomol. Chem., 2018, 16, 3576 3583 
6. Yo

9. Copies of NMR Spectra 
$\mathrm{CDCl}_{3}, 400 \mathrm{MHz}$
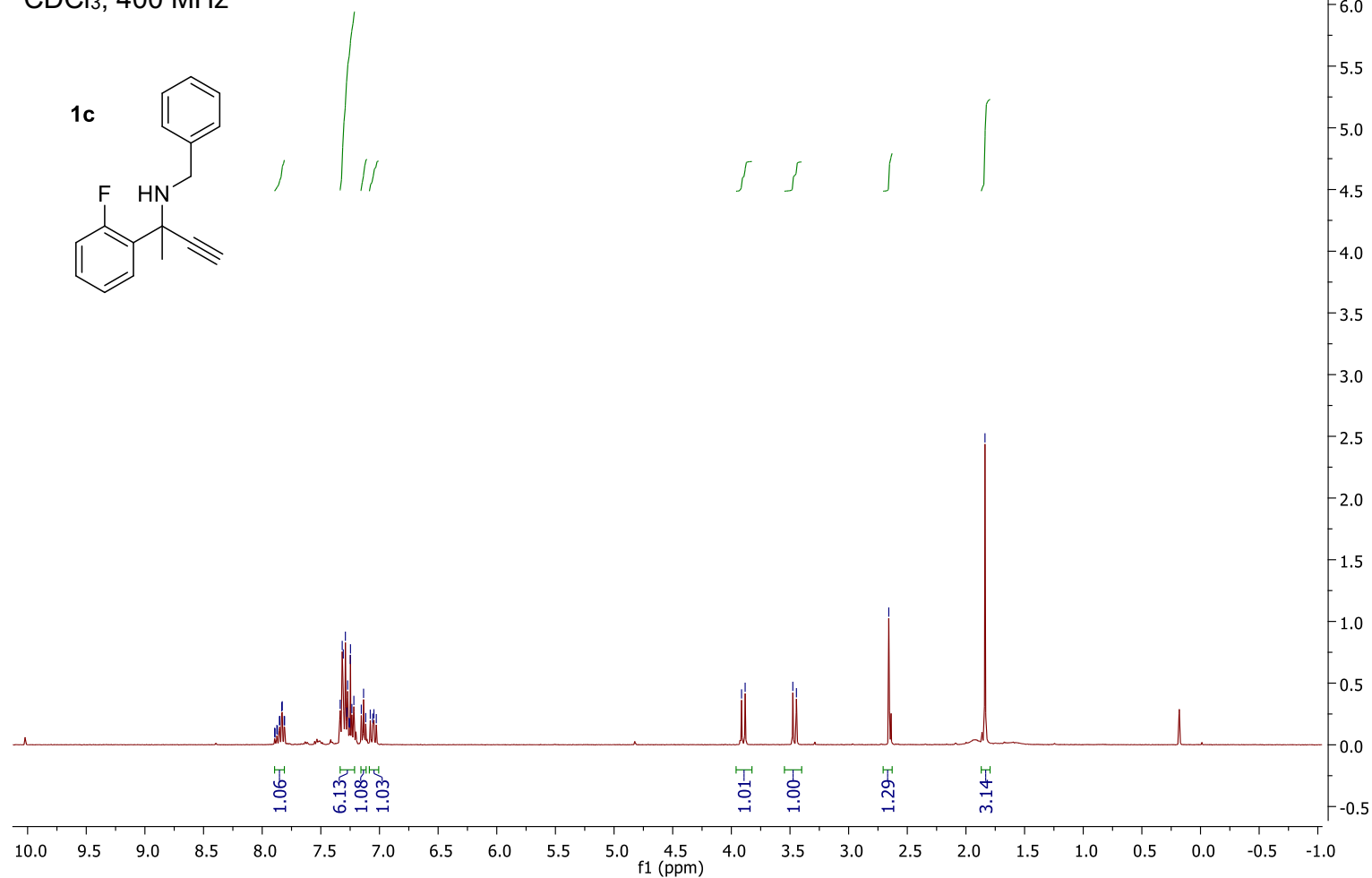

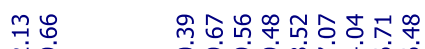

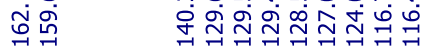

$\hat{\imath}$

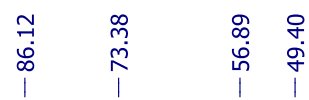

‡i:

$\mathrm{CDCl}_{3}, 101 \mathrm{MHz}$

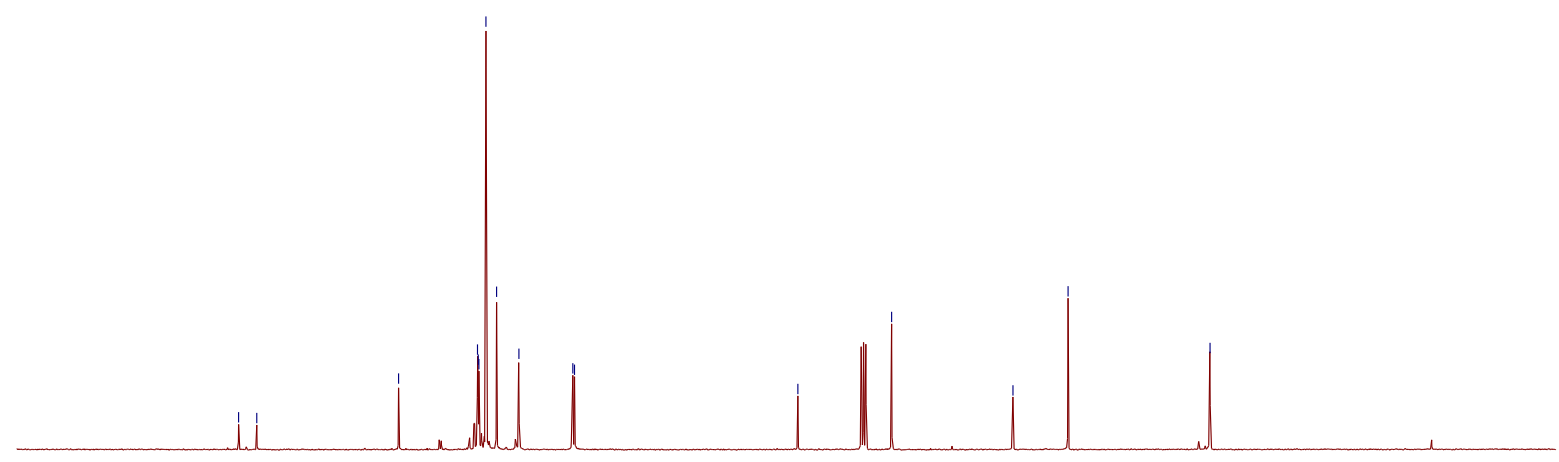

$\begin{array}{lllllllllllllllllllll}190 & 180 & 170 & 160 & 150 & 140 & 130 & 120 & 110 & 100 & \underset{f 1}{90}(\mathrm{ppm}) & 80 & 70 & 60 & 50 & 40 & 30 & 20 & 10 & 0 & -10\end{array}$ 


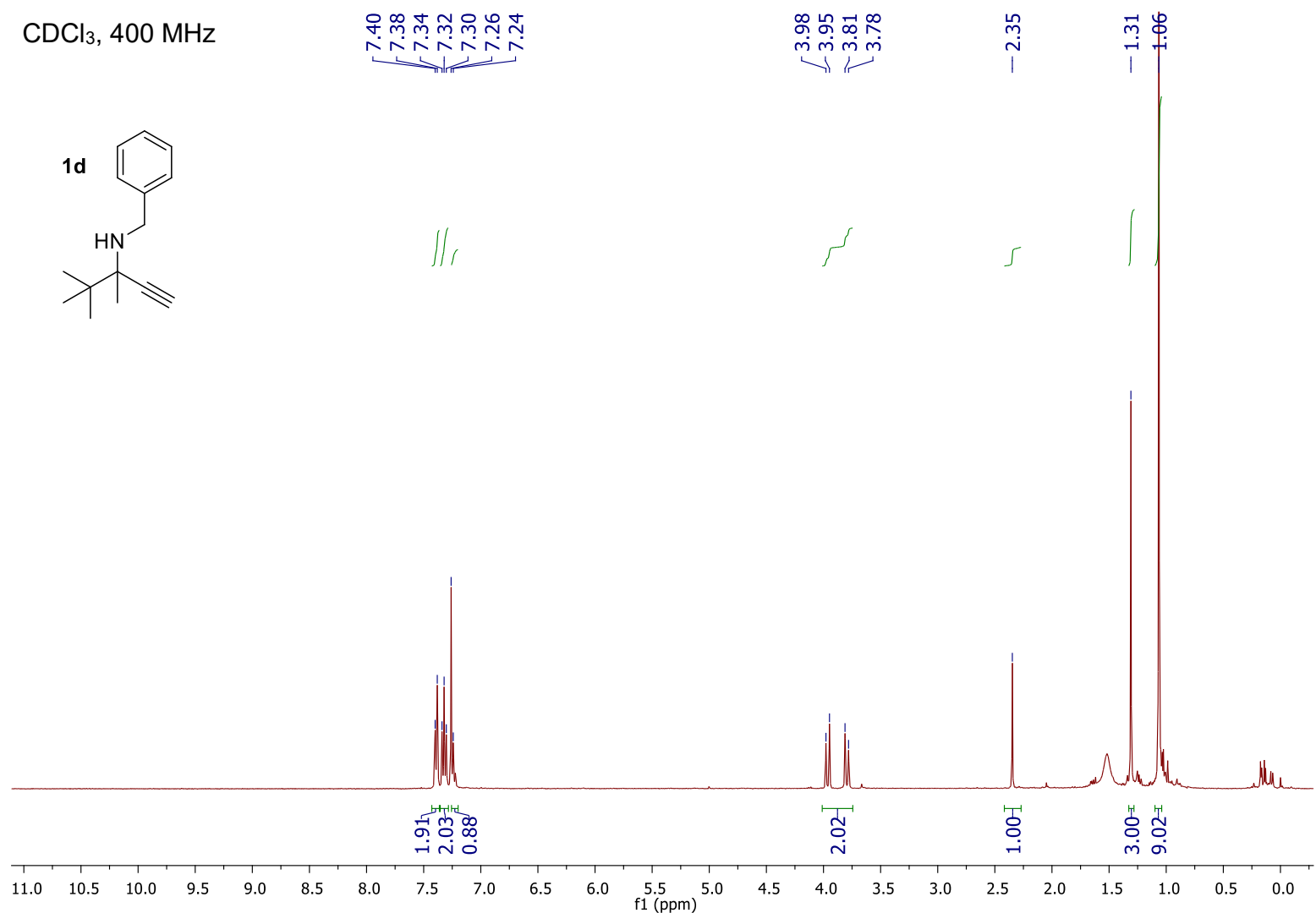

$\mathrm{CDCl}_{3}, 101 \mathrm{MHz}$

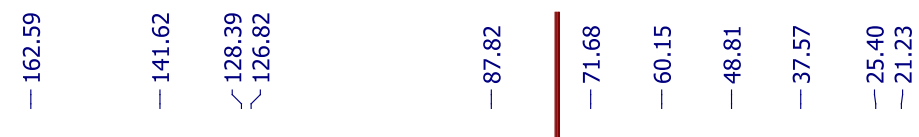

$\begin{array}{llllllllllllllllllllllllllllllllll}220 & 210 & 200 & 190 & 180 & 170 & 160 & 150 & 140 & 130 & 120 & 110 & 100 & 100 & 80 & 70 & 60 & 50 & 40 & 30 & 20 & 10 & 0 & -10 & -20\end{array}$ 


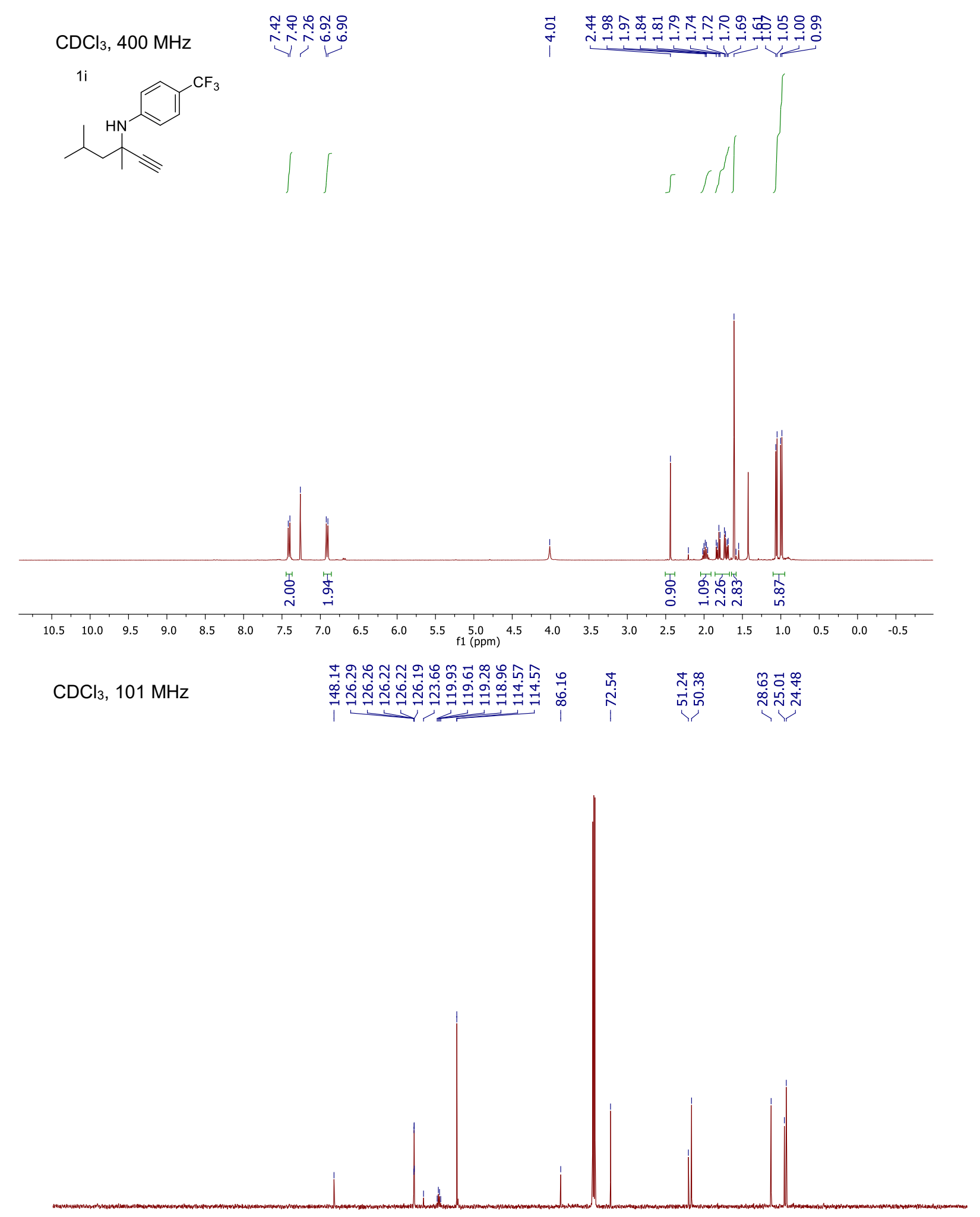

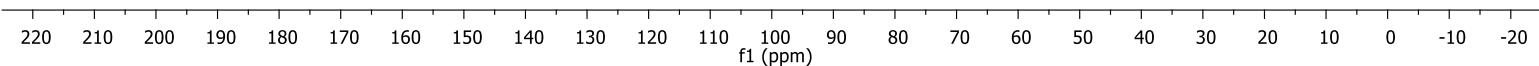


$\mathrm{CDCl}_{3}, 400 \mathrm{MHz}$

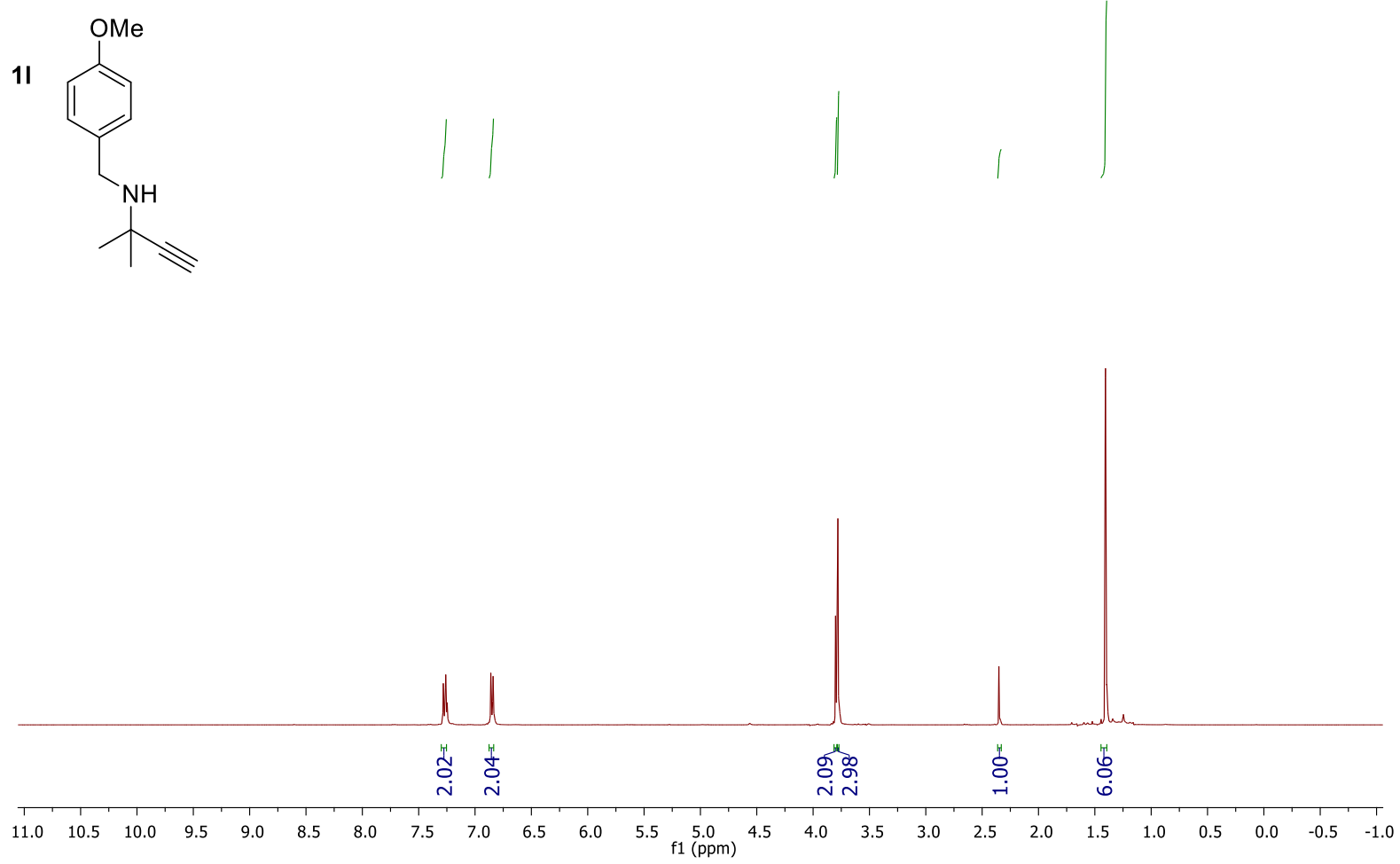

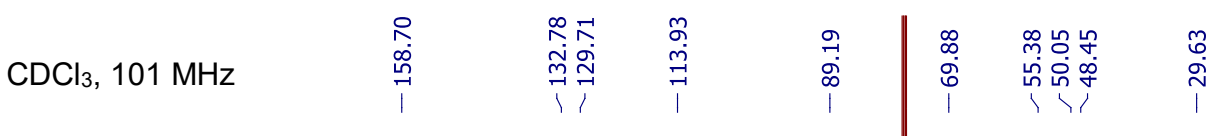

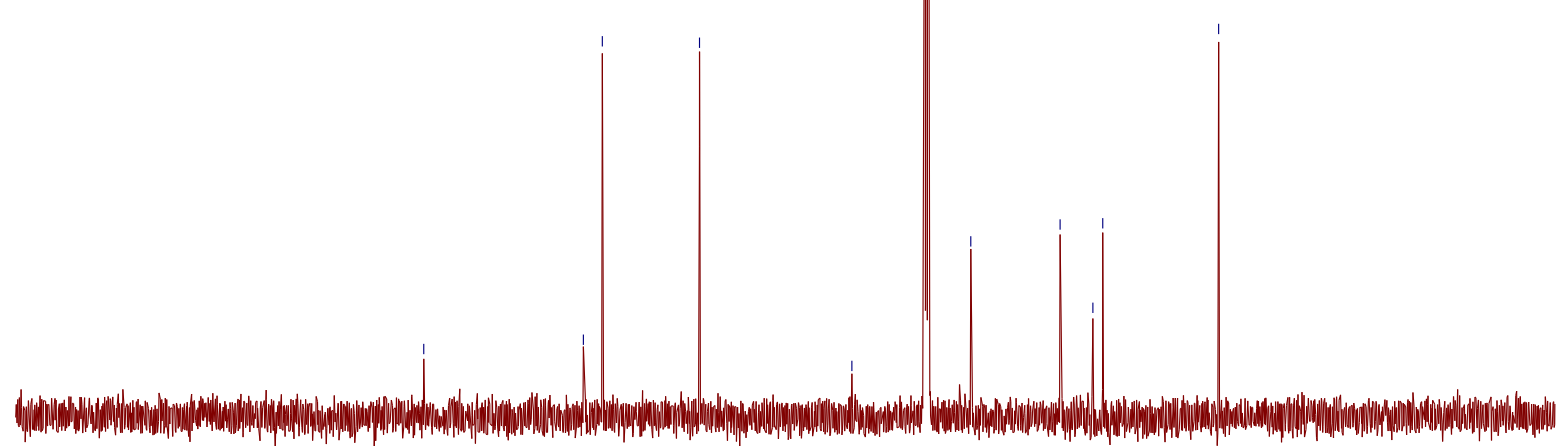

$\begin{array}{lllllllllllllllllllllllll}220 & 210 & 200 & 190 & 180 & 170 & 160 & 150 & 140 & 130 & 120 & 110 & \begin{array}{ll}100 \\ \mathrm{f} 1(\mathrm{ppm})\end{array} & 90 & 80 & 70 & 60 & 50 & 40 & 30 & 20 & 10 & 0 & -10 & -20\end{array}$ 


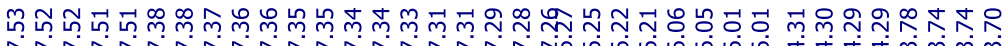

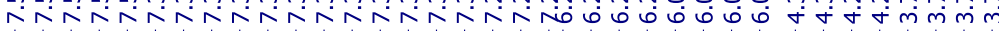
$\mathrm{CDCl}_{3}, 400 \mathrm{MHz}$
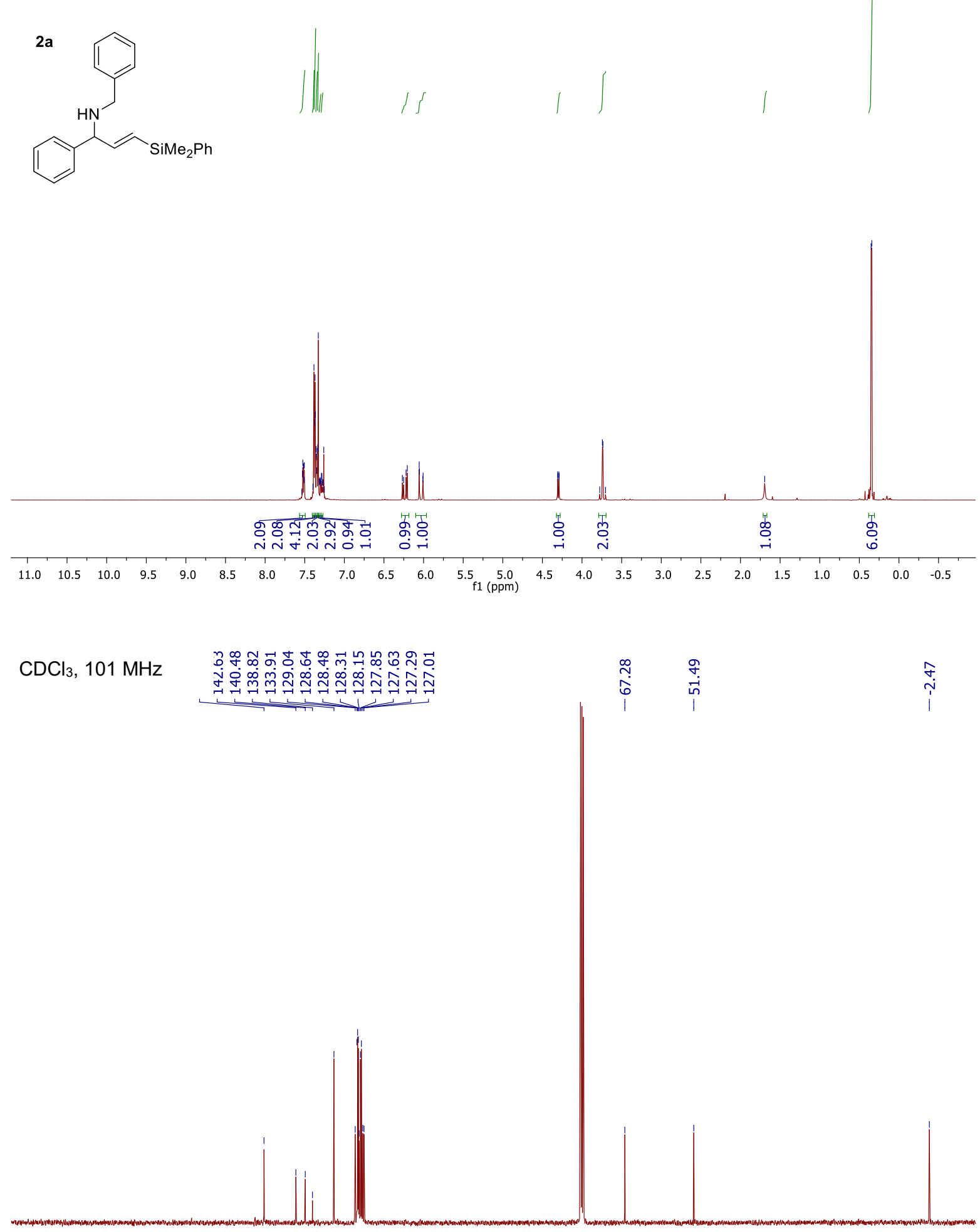

$\begin{array}{llllllllllllllllllllll}200 & 190 & 180 & 170 & 160 & 150 & 140 & 130 & 120 & 110 & \underset{f 1}{100}(\mathrm{ppm}) & 90 & 80 & 70 & 60 & 50 & 40 & 30 & 20 & 10 & 0 & -10\end{array}$ 


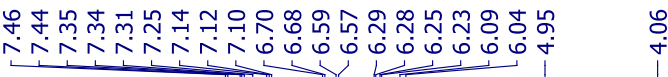

$\mathrm{CDCl}_{3}, 400 \mathrm{MHz}$

2b
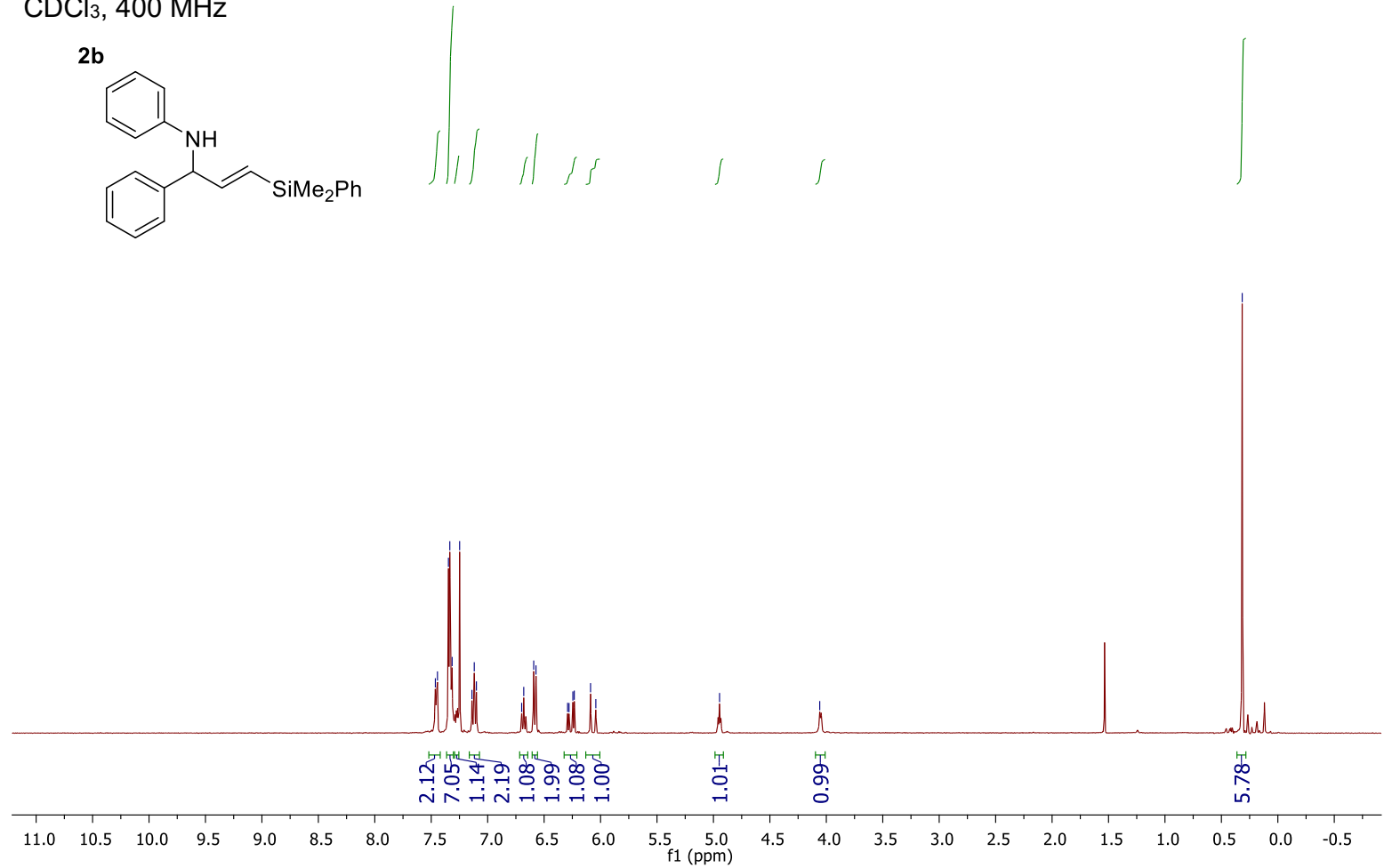

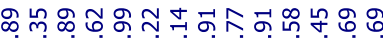

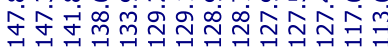

ஸู

خ̃

$\mathrm{CDCl}_{3}, 101 \mathrm{MHz}$

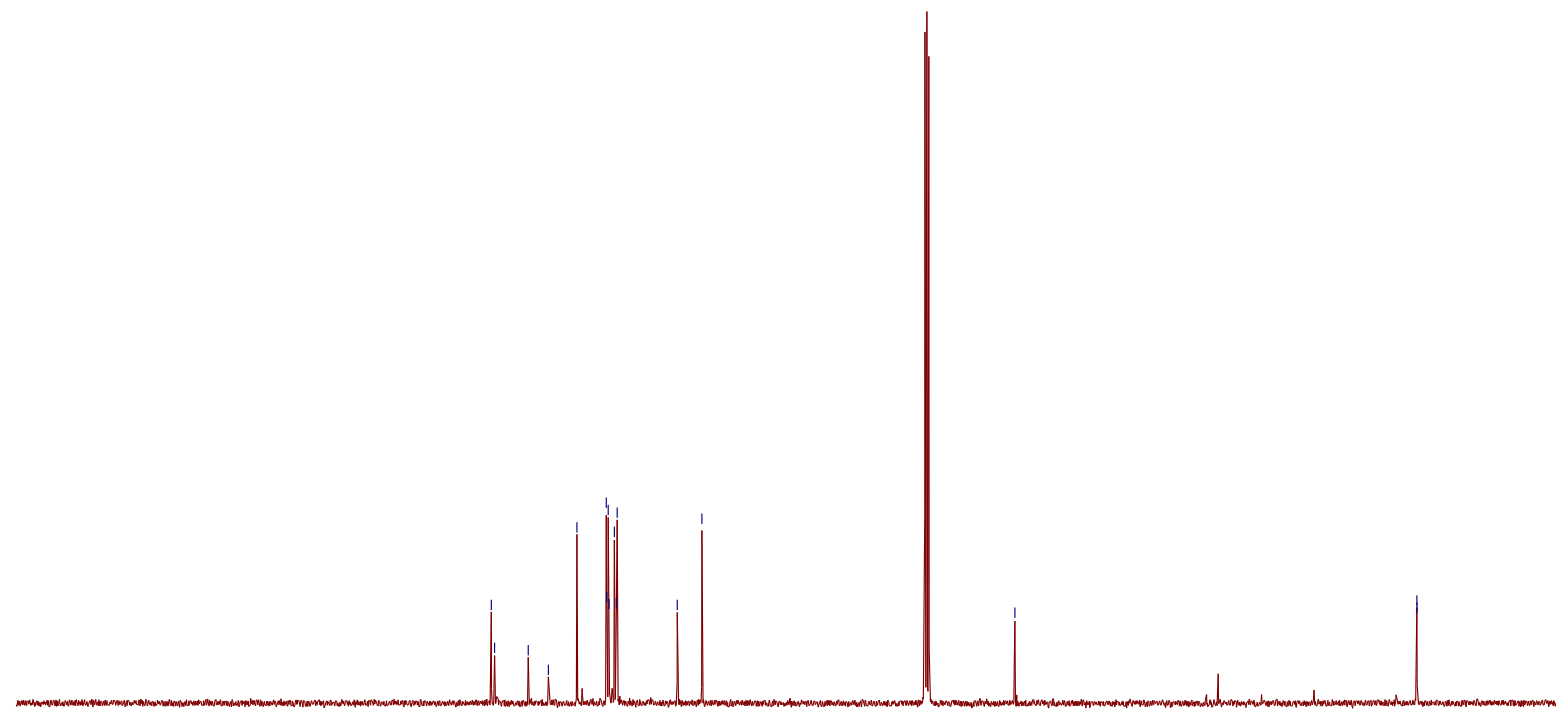

$\begin{array}{llllllllllllllllllllllllllllll}220 & 210 & 200 & 190 & 180 & 170 & 160 & 150 & 140 & 130 & 120 & 110 & 100 & 90 & 80 & 70 & 60 & 50 & 40 & 30 & 20 & 10 & 0 & -10 & -20\end{array}$ 


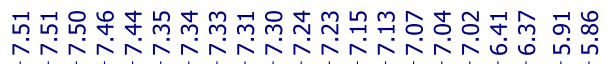

$\mathrm{CDCl}_{3}, 400 \mathrm{MHz}$

นึํำ ํํำ

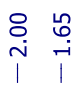

$\stackrel{m}{m}$

nim

2c
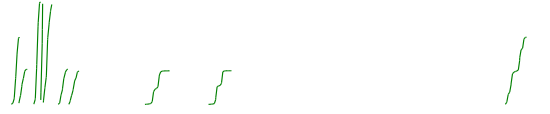

$\int$<smiles>CCNC(C)(/C=C/[AsH2]c1ccccc1)c1ccccc1F</smiles>
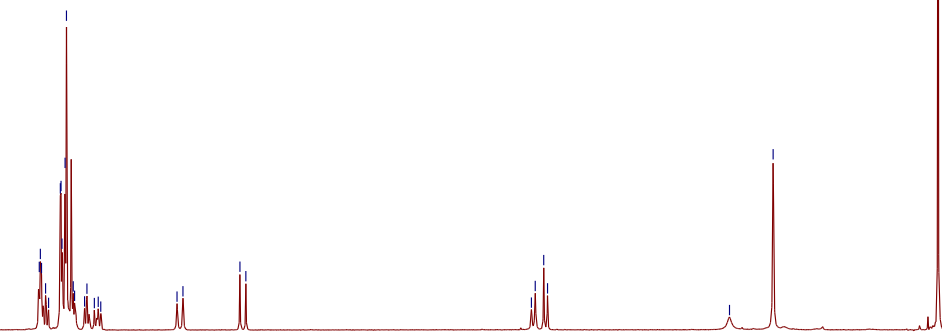

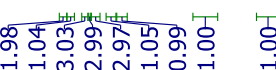

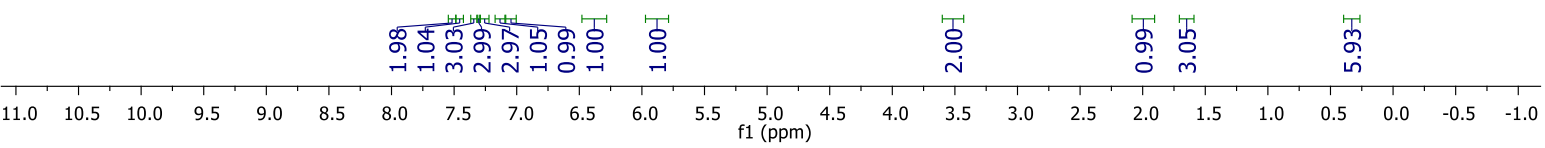

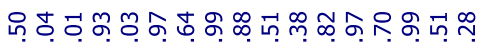

$\mathrm{CDCl}_{3}, 101 \mathrm{MHz}$

ஸ્่

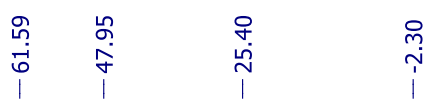

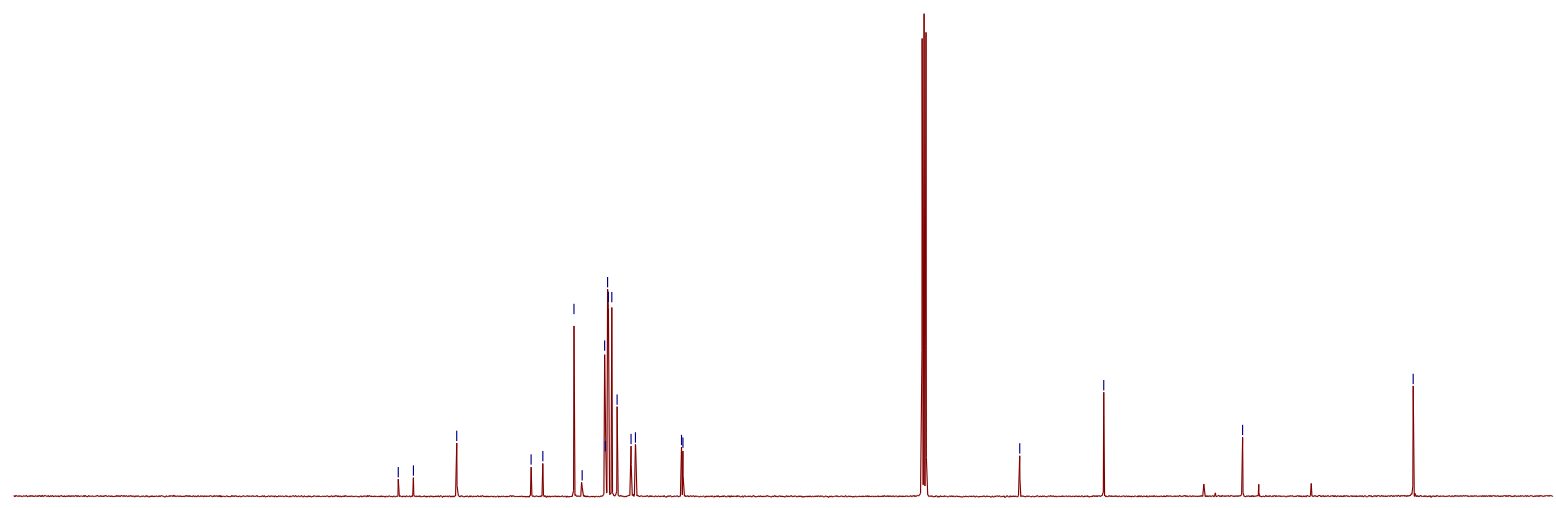

$\begin{array}{lllllllllllllllllllllllll}220 & 210 & 200 & 190 & 180 & 170 & 160 & 150 & 140 & 130 & 120 & 110 & 100 & 90 & 80 & 70 & 60 & 50 & 40 & 30 & 20 & 10 & 0 & -10 & -20\end{array}$ 


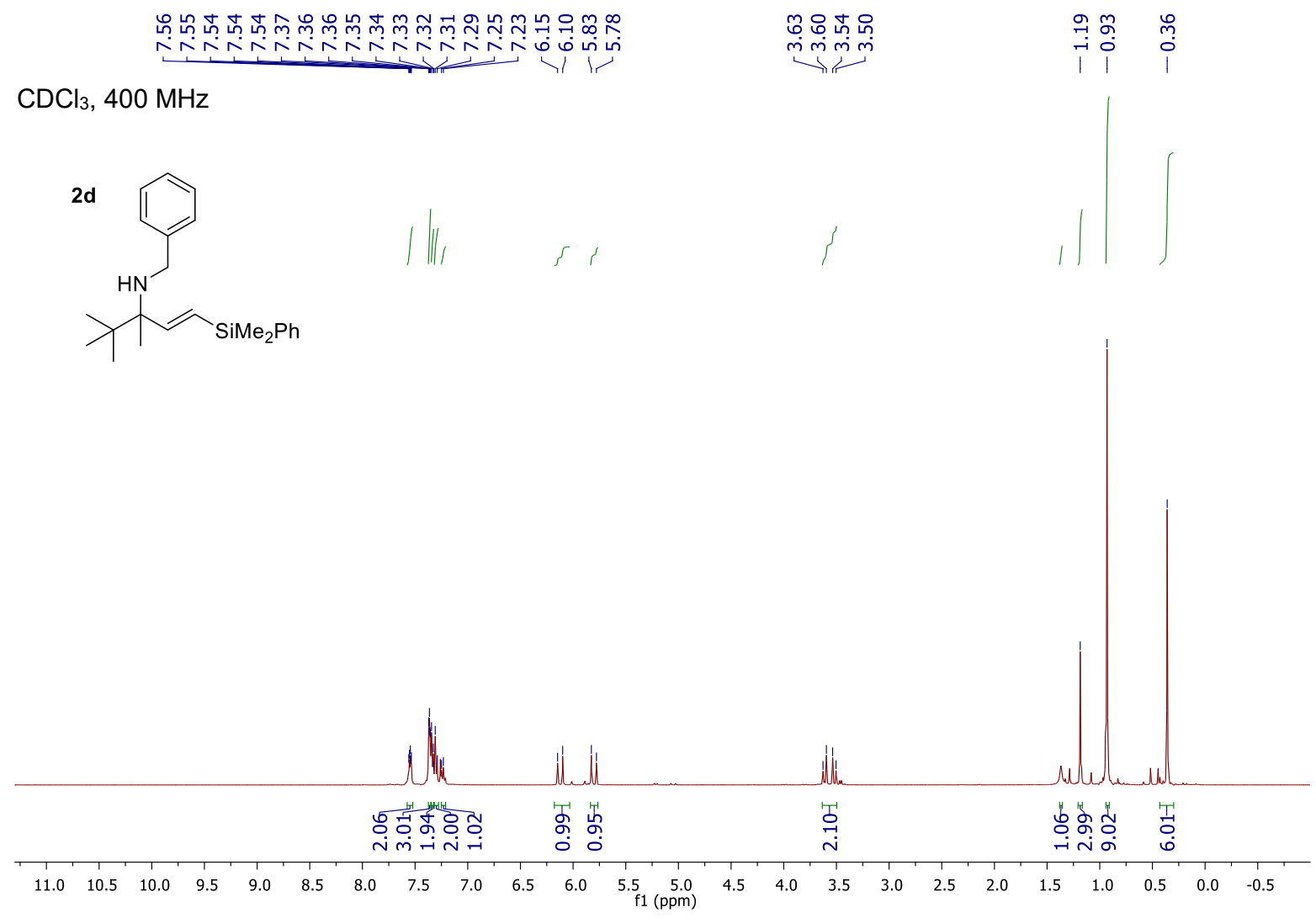

$\mathrm{CDCl}_{3}, 101 \mathrm{MHz} \quad$ 年

$\begin{array}{lllllllllllllllllllllllllllllll}220 & 210 & 200 & 190 & 180 & 170 & 160 & 150 & 140 & 130 & 120 & 110 & 100 & 100 & 80 & 70 & 60 & 50 & 40 & 30 & 20 & 10 & 0 & -10 & -20\end{array}$ 

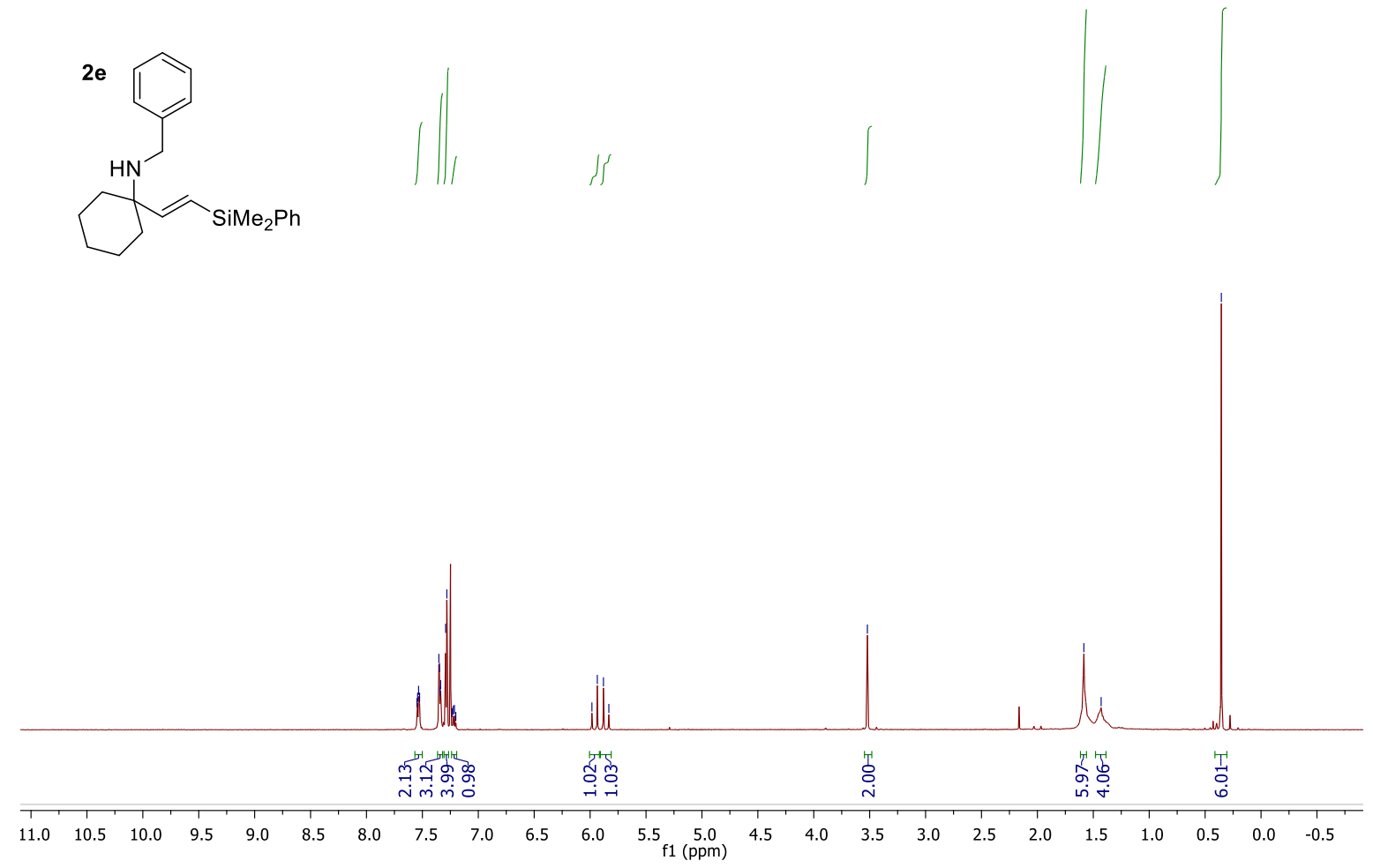

$\mathrm{CDCl}_{3}, 101 \mathrm{MHz}$

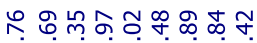

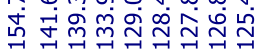

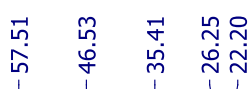

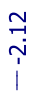

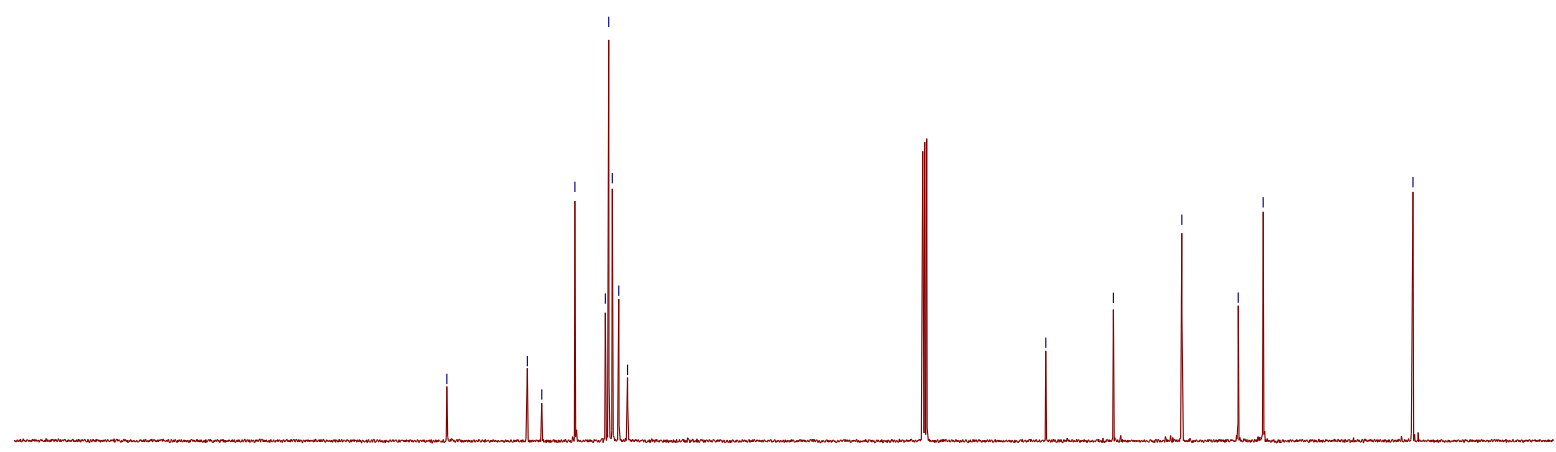

$\begin{array}{lllllllllllllllllllllllll}220 & 210 & 200 & 190 & 180 & 170 & 160 & 150 & 140 & 130 & 120 & 110 & 100 & 100 & 80 & 70 & 60 & 50 & 40 & 30 & 20 & 10 & 0 & -10 & -20\end{array}$ 


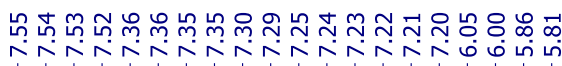

$\mathrm{CDCl}_{3}, 400 \mathrm{MHz}$

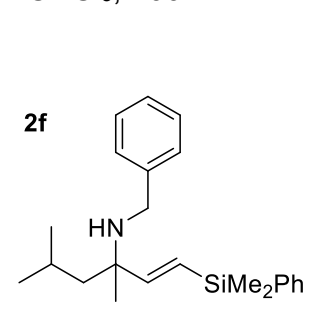

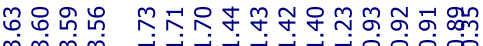

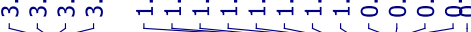
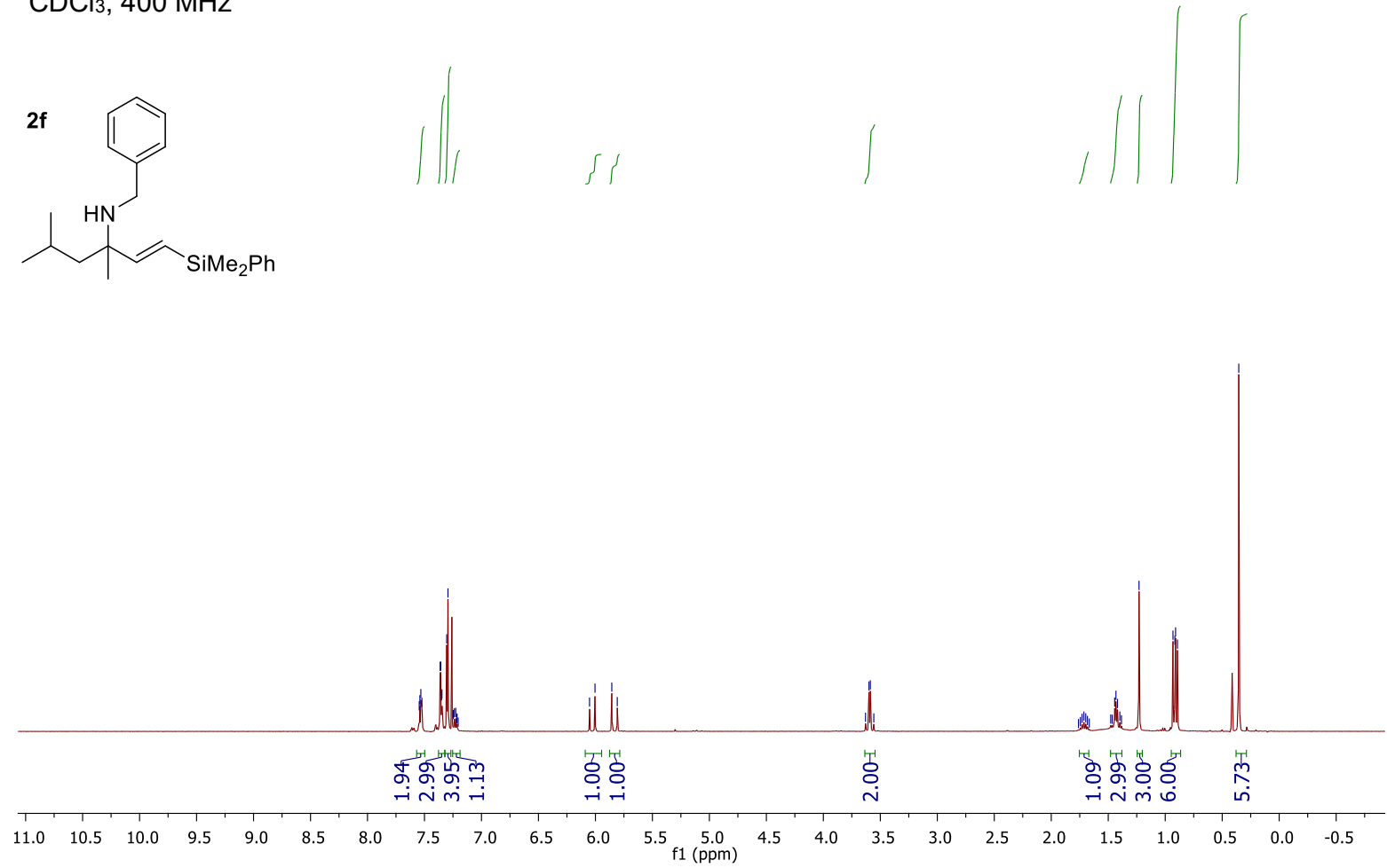

$\mathrm{CDCl}_{3}, 101 \mathrm{MHz}$

\section{ㅅำ}

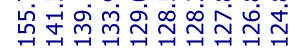

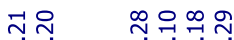

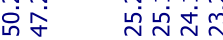

$\stackrel{\vec{N}}{i}$

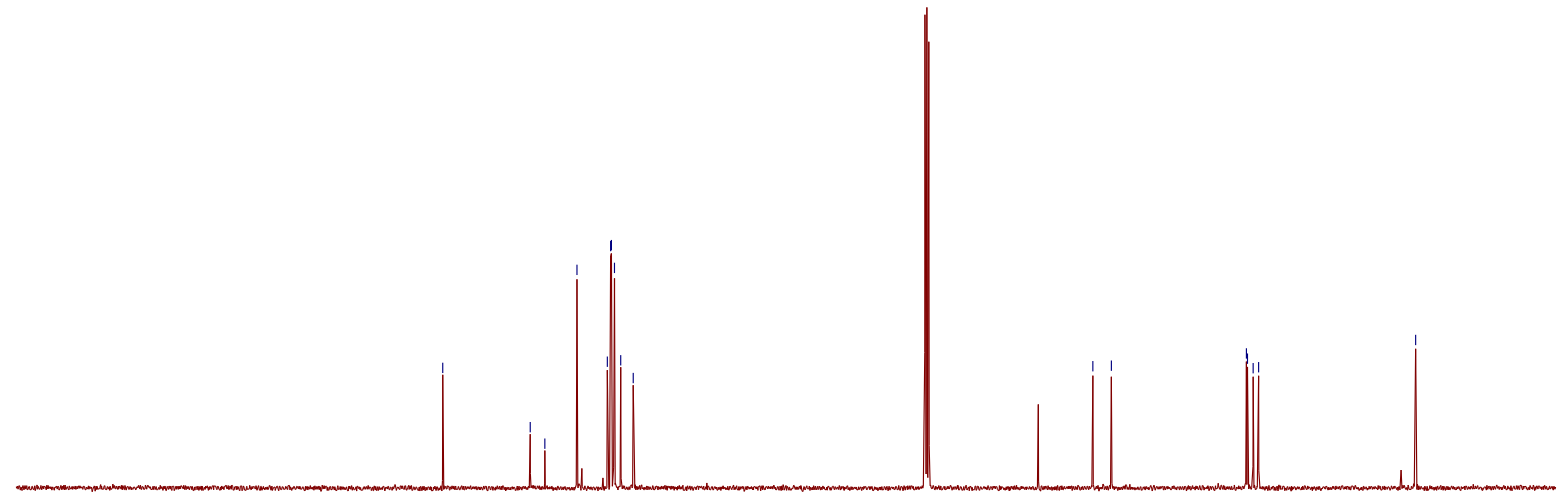

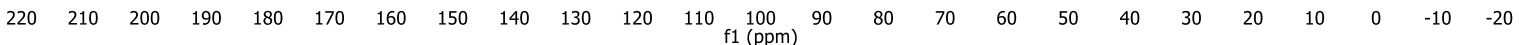


$\mathrm{CDCl}_{3}, 400 \mathrm{MHz}$

2g<smiles>CC(C=COc1ccccc1)(Nc1ccccc1)c1ccccc1</smiles>
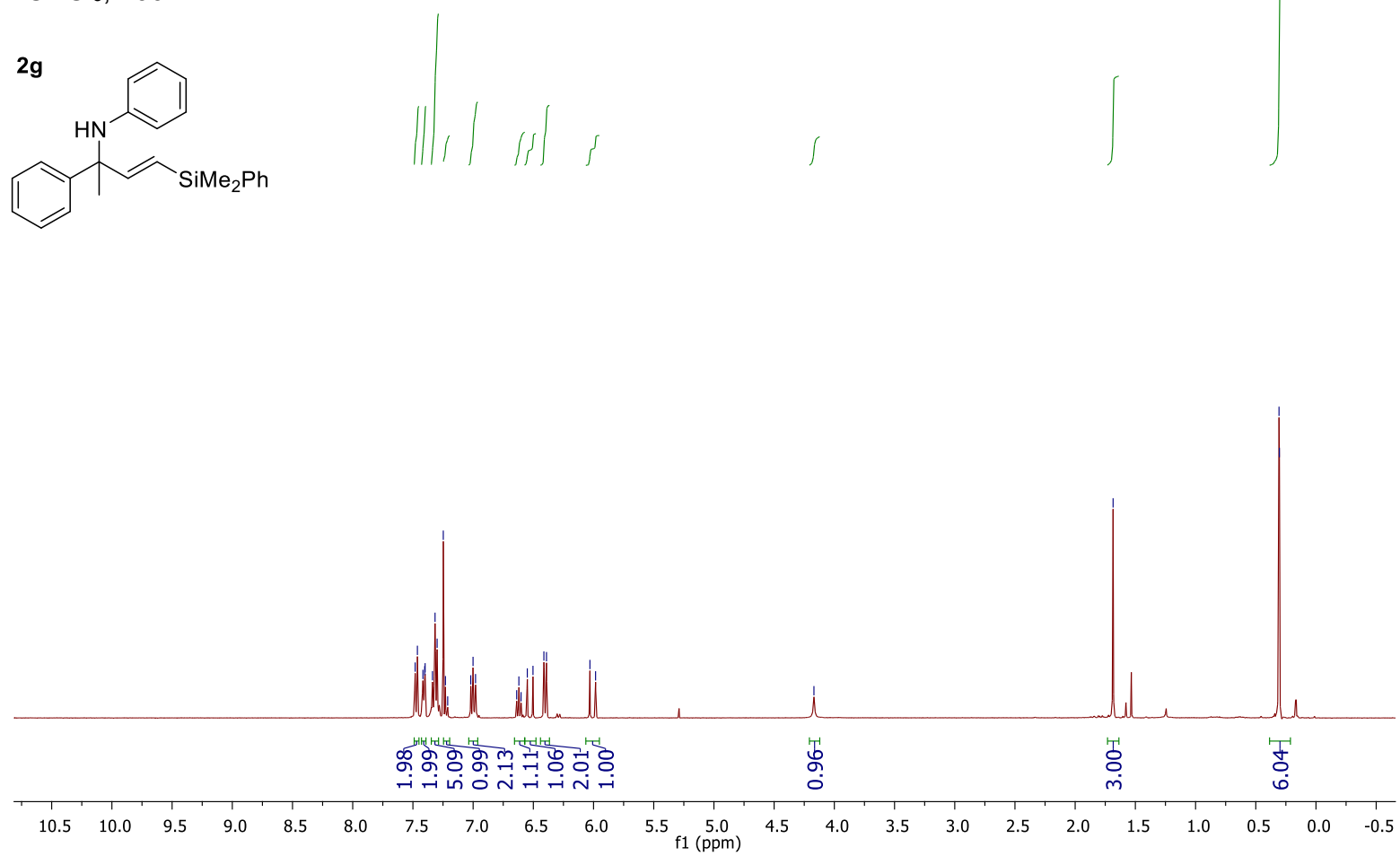

$\mathrm{CDCl}_{3}, 101 \mathrm{MHz}$ 
<smiles>Cc1ccc(C(C)(/C=C/Sc2ccccc2)Nc2ccccc2)cc1</smiles>
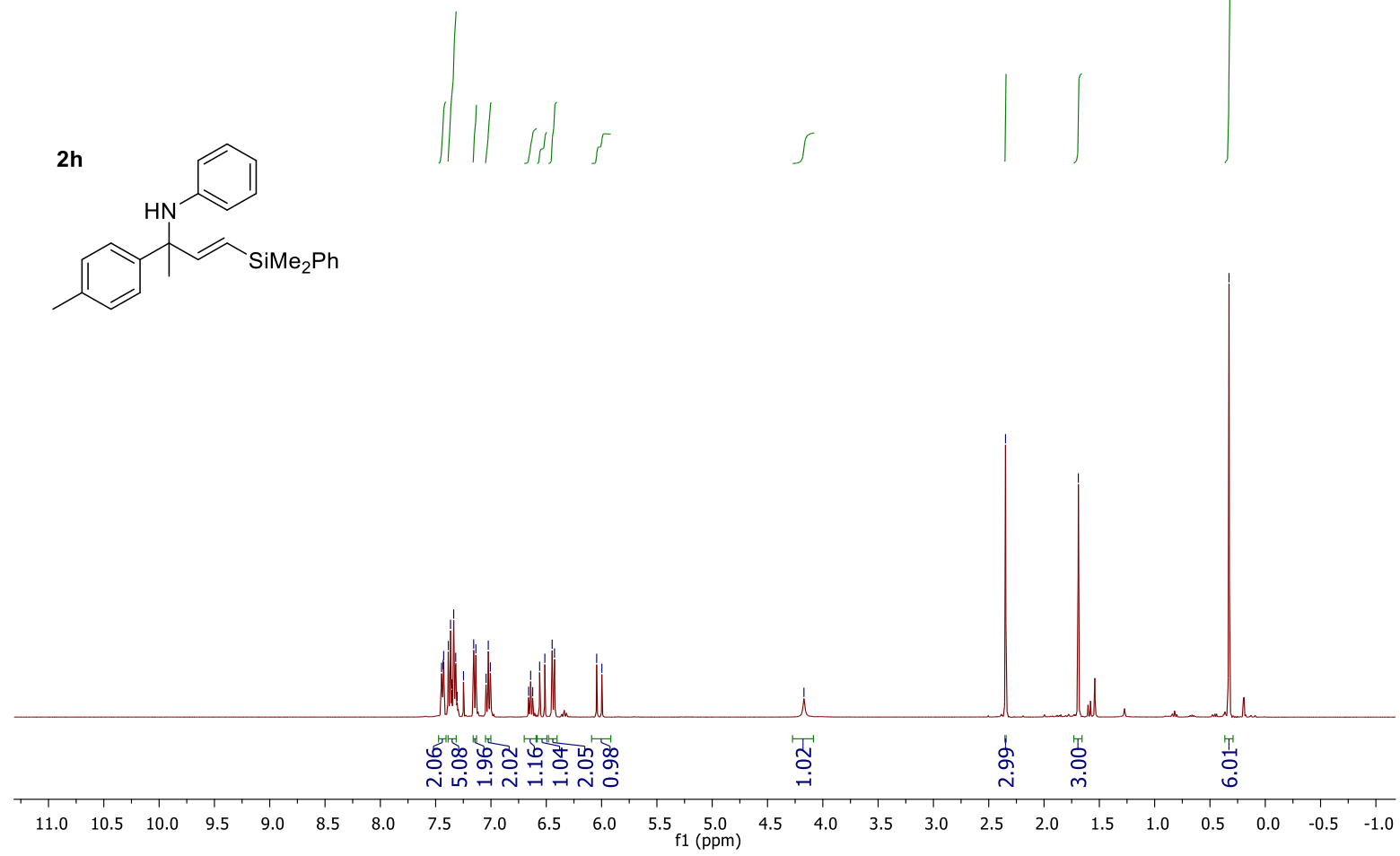

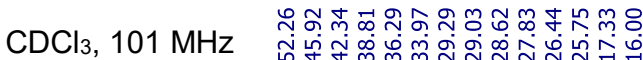
نำ

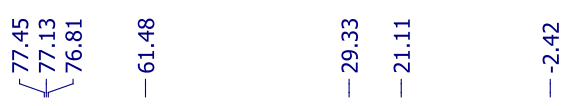

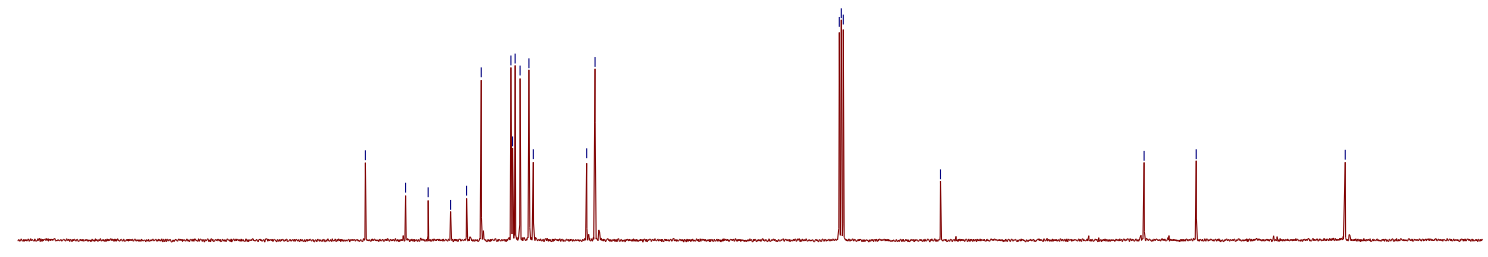

$\begin{array}{llllllllllllllllllllllllllllll}200 & 190 & 180 & 170 & 160 & 150 & 140 & 130 & 120 & 110 & 100 & 90 & 80 & 70 & 60 & 50 & 40 & 30 & 20 & 10 & 0 & -10 & -20\end{array}$ 


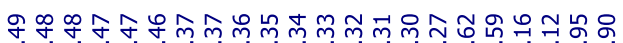

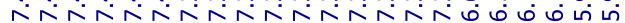

$\mathrm{CDCl}_{3}, 400 \mathrm{MHz}$

2i<smiles>CC(C)CC(C)(/C=C/Nc1ccccc1)Nc1ccc(C(F)(F)F)cc1</smiles>

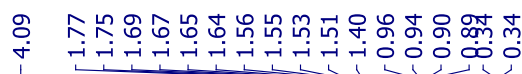

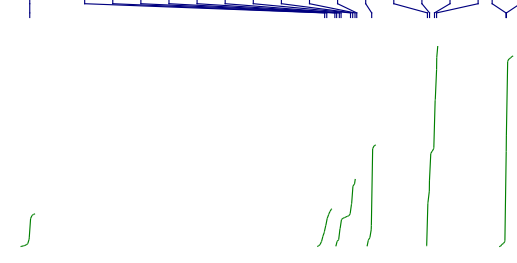

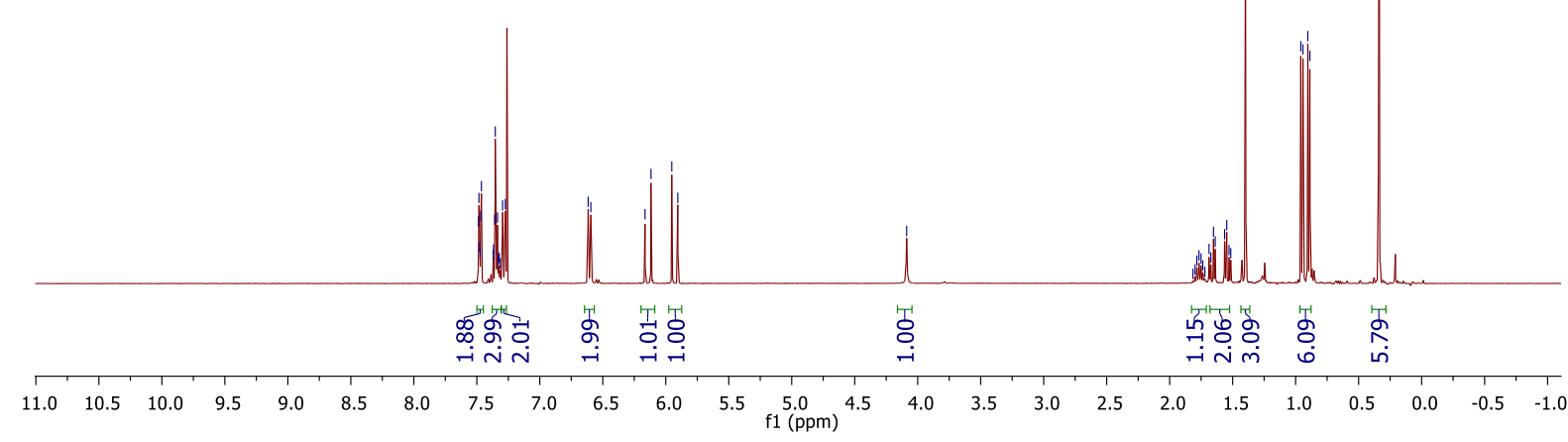

$\mathrm{CDCl}_{3}, 101 \mathrm{MHz}$

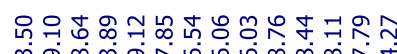

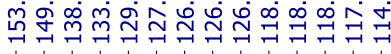

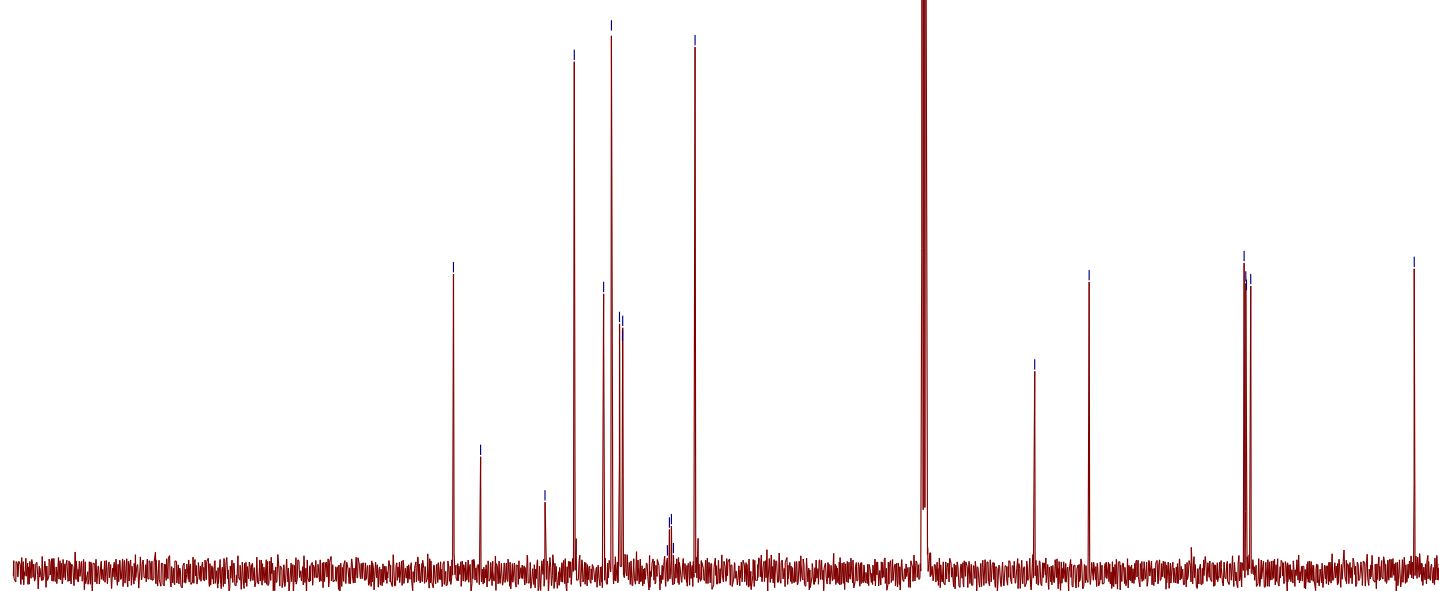

공

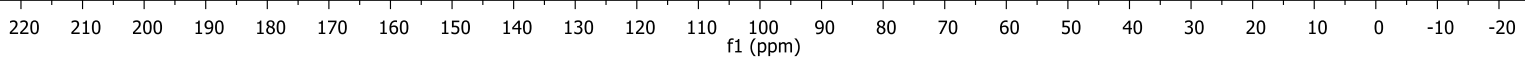



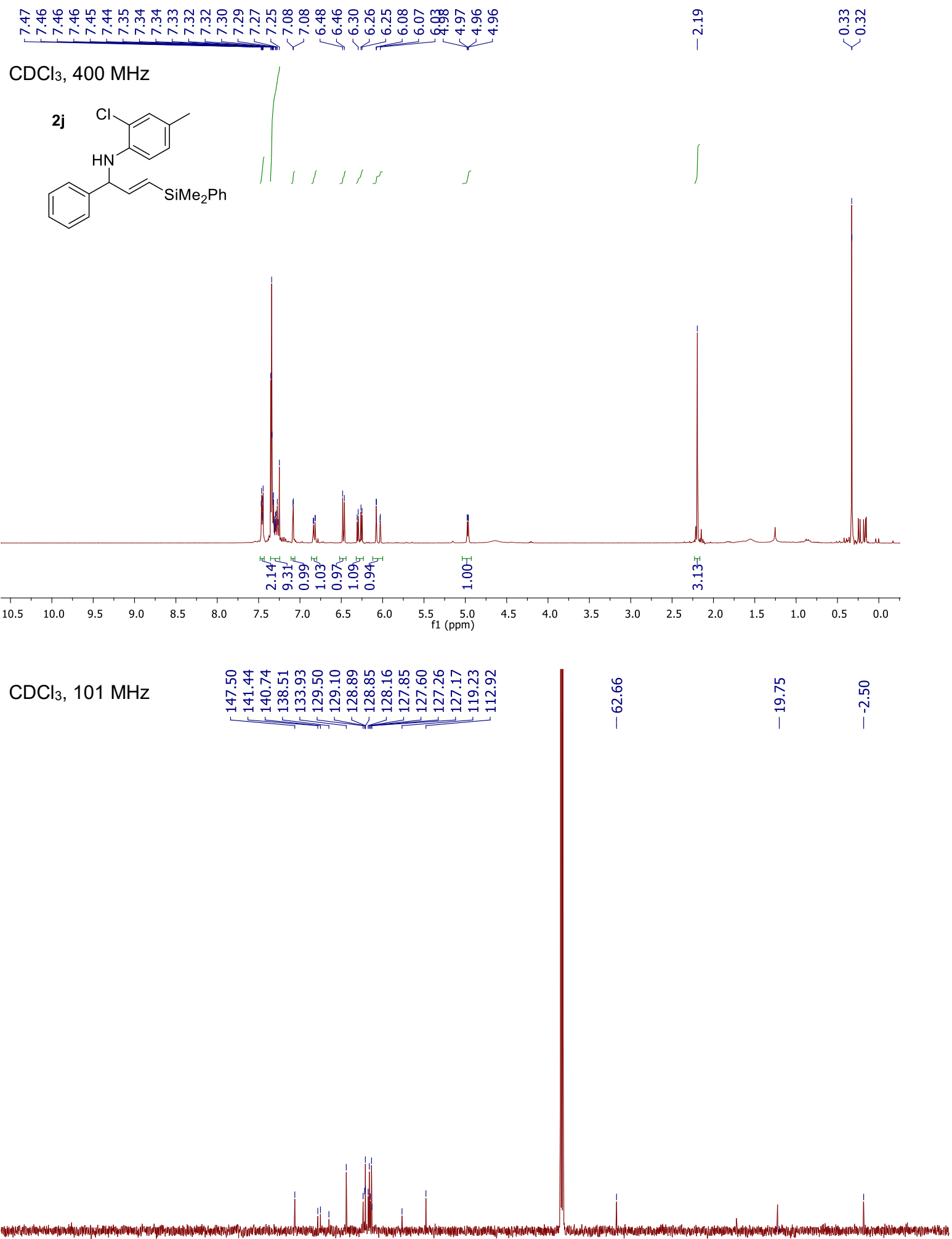

$\begin{array}{llllllllllllllllllllllllllllll}220 & 210 & 200 & 190 & 180 & 170 & 160 & 150 & 140 & 130 & 120 & 110 & 100 & 90 & 80 & 70 & 60 & 50 & 40 & 30 & 20 & 10 & 0 & -10 & -20\end{array}$ 

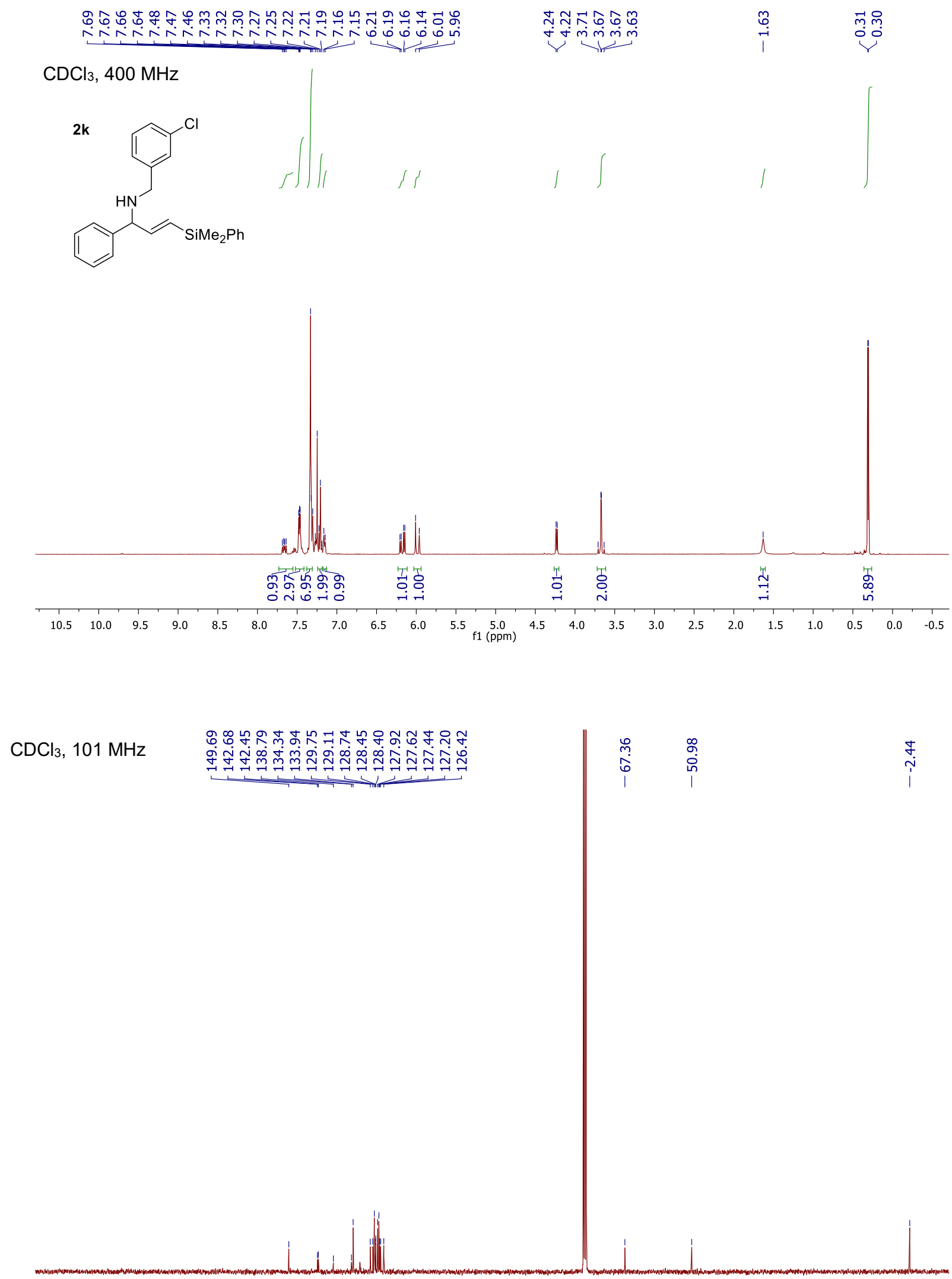

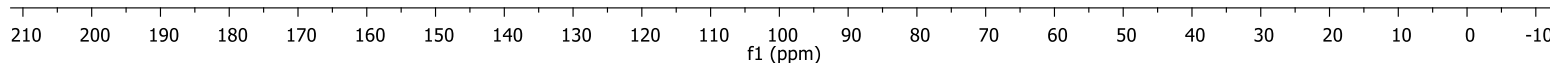




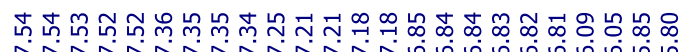
กักง

$\mathrm{CDCl}_{3}, 400 \mathrm{MHz}$
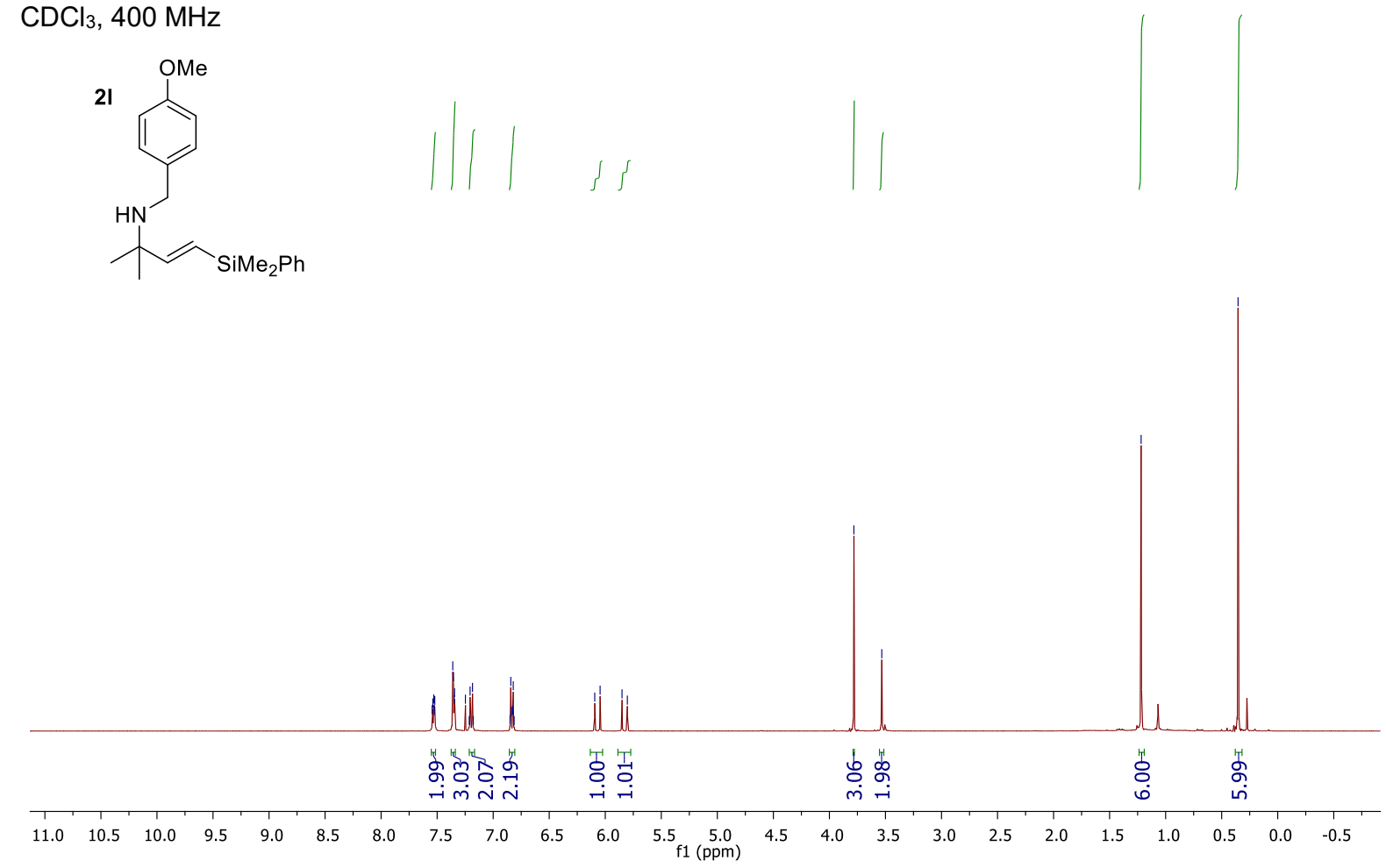

$\mathrm{CDCl}_{3}, 101 \mathrm{MHz}$

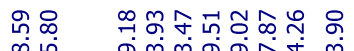

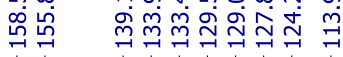

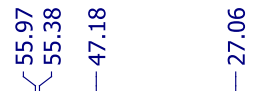

$\stackrel{\stackrel{+}{i}}{i}$
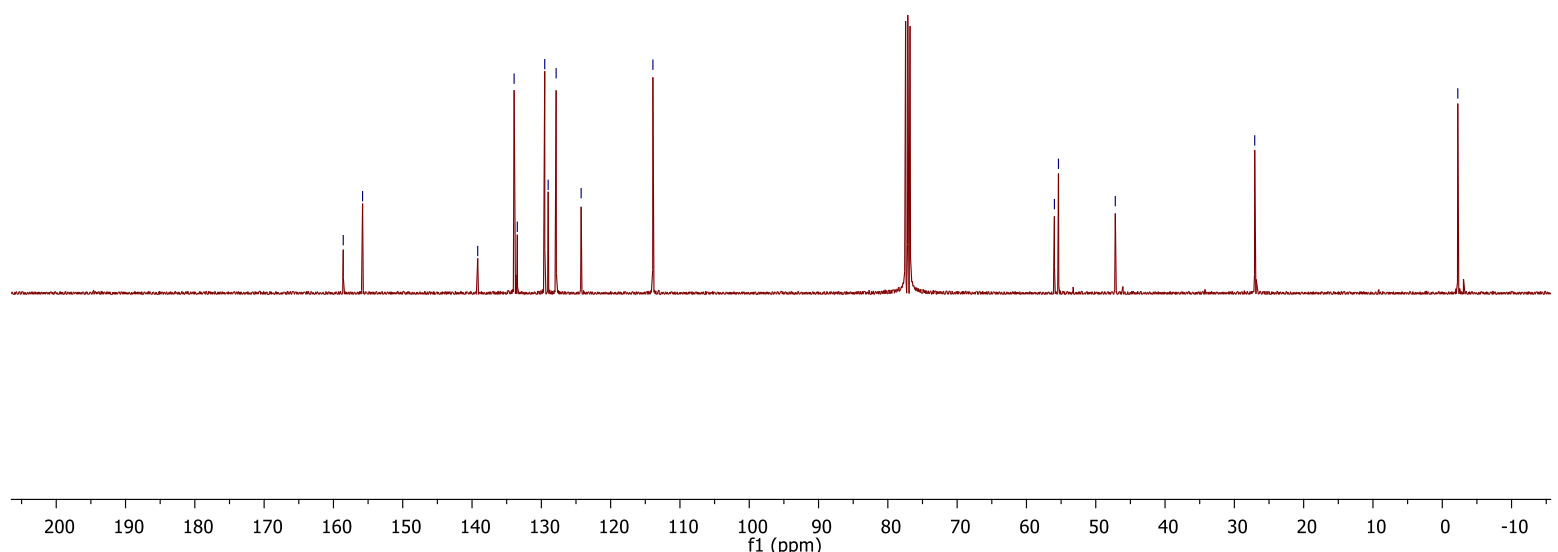
$\mathrm{CDCl}_{3}, 400 \mathrm{MHz}$

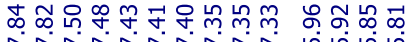

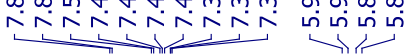

$\stackrel{\substack{n \\ i}}{i}$<smiles>CC(C)(/C=C/c1ccccc1)NS(=O)(=O)c1ccccc1</smiles><smiles>C1#CCCC1</smiles>

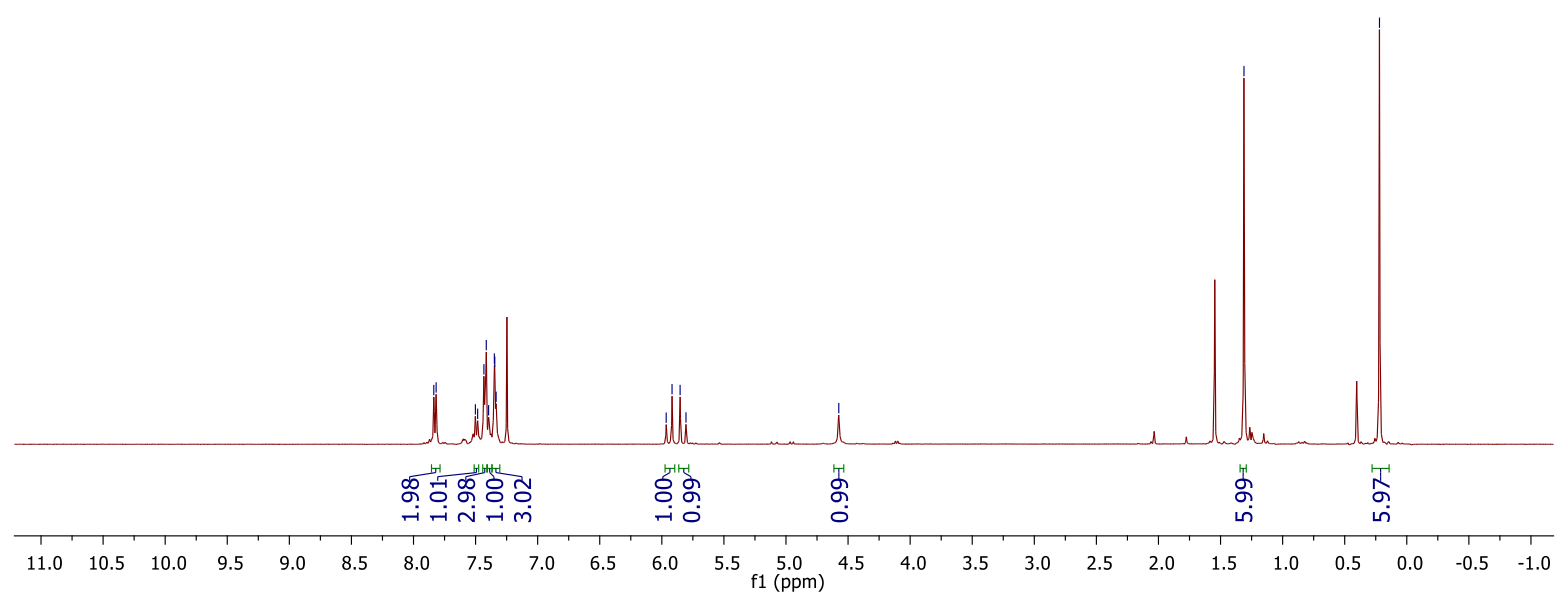

$\mathrm{CDCl}_{3}, 101 \mathrm{MHz}$

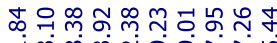

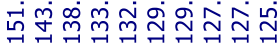

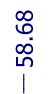

$\stackrel{\substack{\infty \\ \infty}}{\sim}$

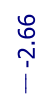

$\begin{array}{lllllllllllllllllllllll}210 & 200 & 190 & 180 & 170 & 160 & 150 & 140 & 130 & 120 & 110 & \begin{array}{c}100 \\ \mathrm{f} 1(\mathrm{ppm})\end{array} & 90 & 80 & 70 & 60 & 50 & 40 & 30 & 20 & 10 & 0 & -10\end{array}$ 


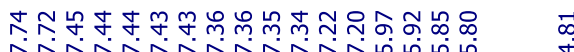

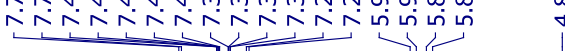

$\stackrel{\overrightarrow{0}}{\dot{+}}$

$\mathrm{CDCl}_{3}, 400 \mathrm{MHz}$

$4 b$<smiles>Cc1ccc(S(=O)(=O)NC(C)(C)/C=C/Sc2ccccc2)cc1</smiles>

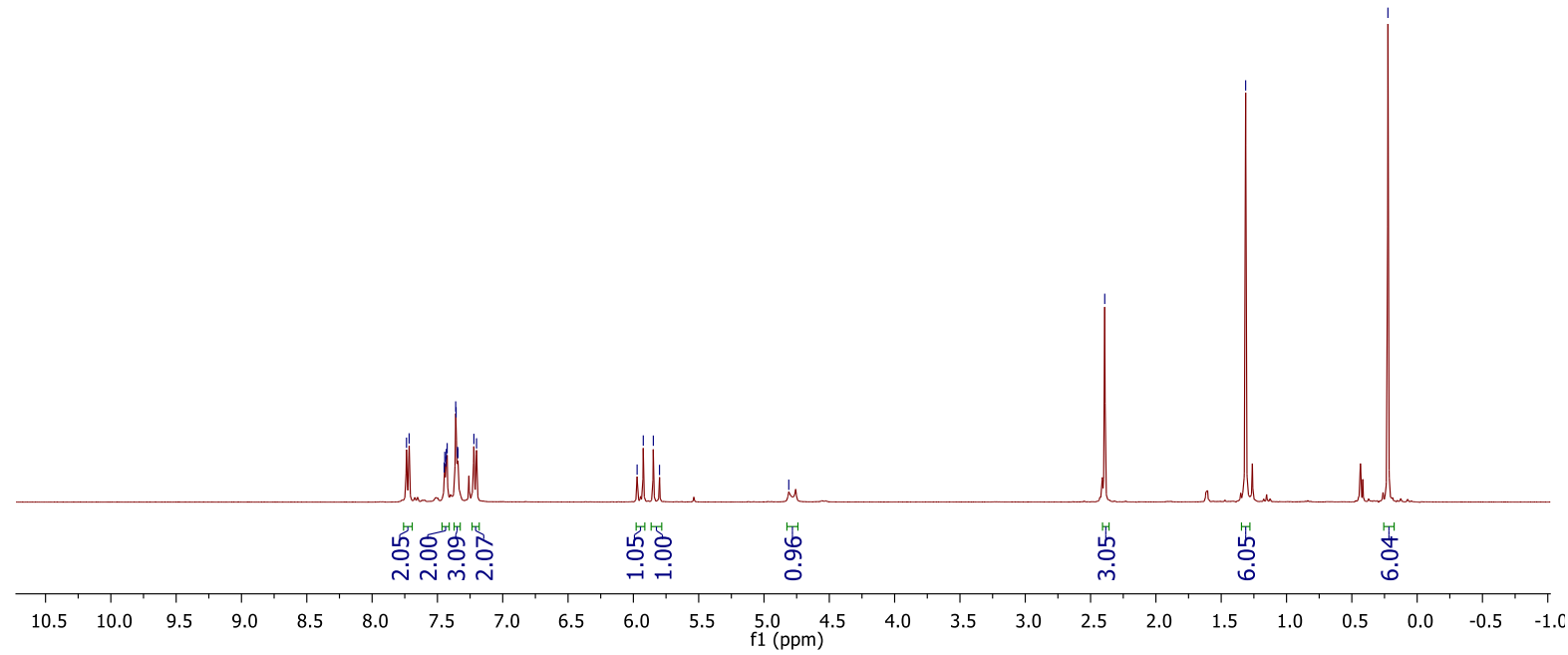

$\mathrm{CDCl}_{3}, 101 \mathrm{MHz}$

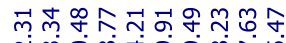

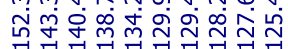

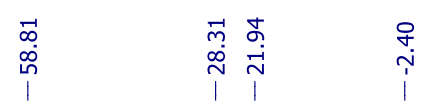

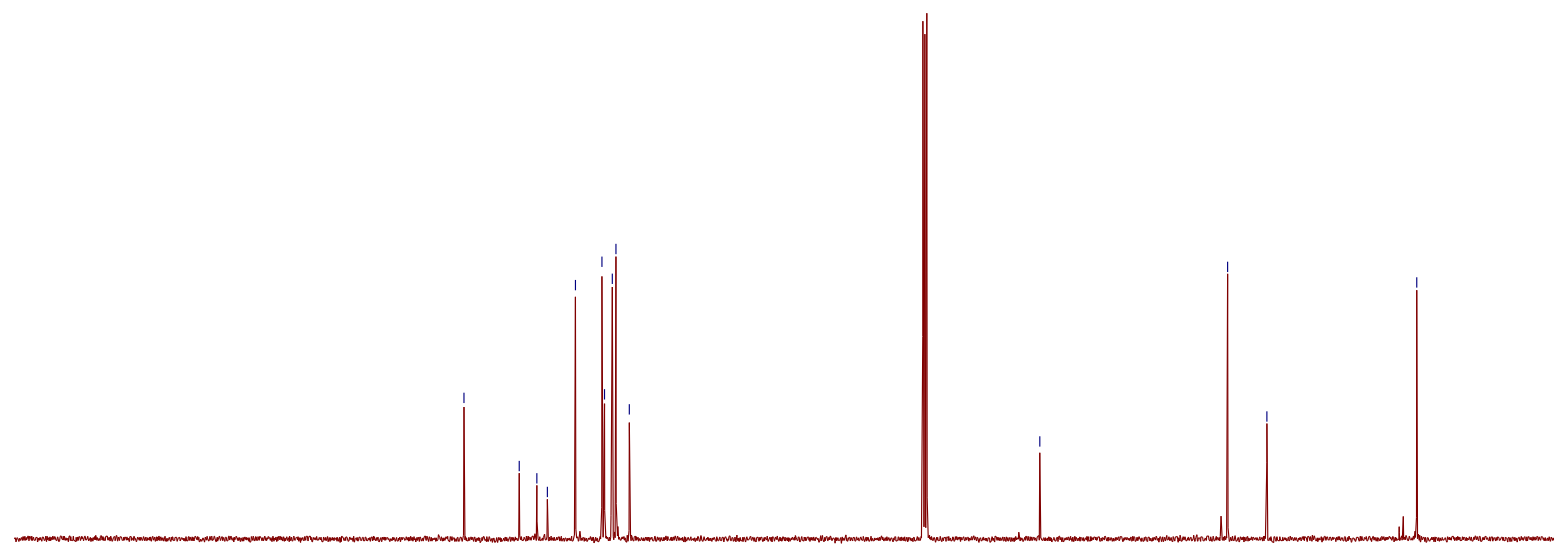

$\begin{array}{llllllllllllllllllllllllll}220 & 210 & 200 & 190 & 180 & 170 & 160 & 150 & 140 & 130 & 120 & 110 & \underset{\mathrm{f} 1}{100}(\mathrm{ppm}) & 90 & 80 & 70 & 60 & 50 & 40 & 30 & 20 & 10 & 0 & -10 & -20\end{array}$ 


\section{تخ}

$\mathrm{CDCl}_{3}, 400 \mathrm{MHz}$
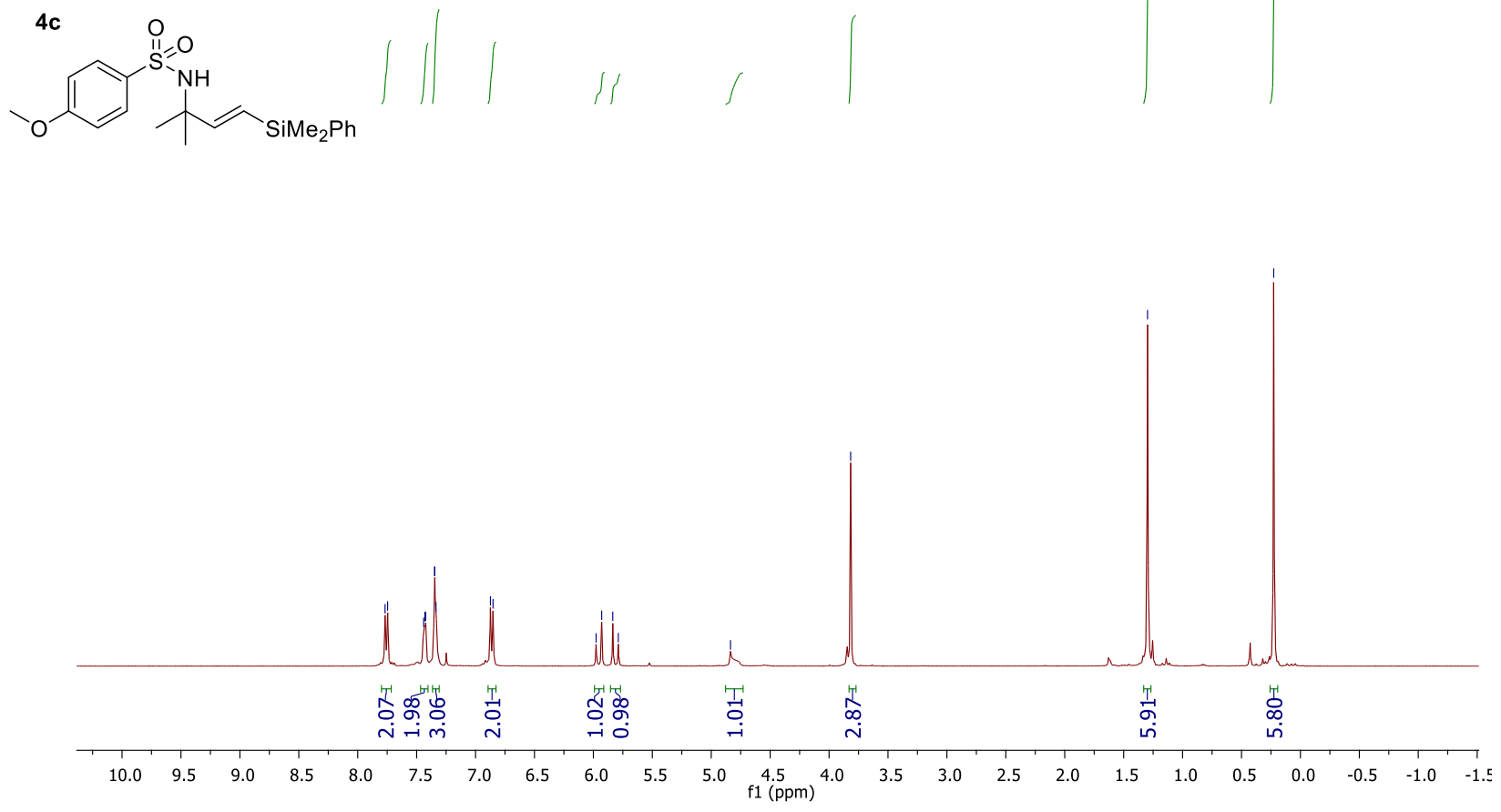

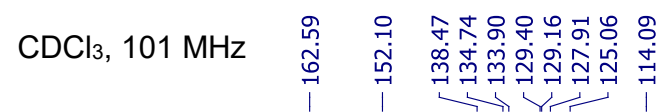

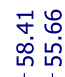

$\stackrel{\infty}{\stackrel{\infty}{\curvearrowright}}$

$\stackrel{\leftrightarrow}{i}$

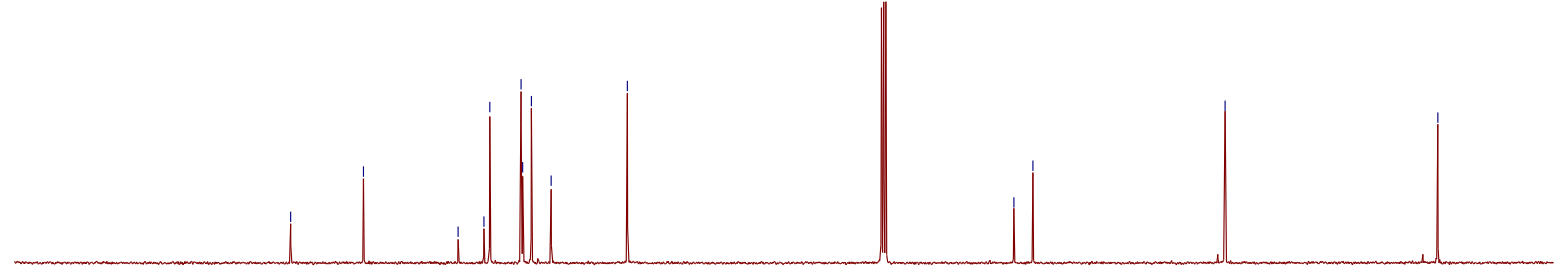

$\begin{array}{lllllllllllllllllllllllllll}200 & 190 & 180 & 170 & 160 & 150 & 140 & 130 & 120 & 110 & 100 & 90 & 80 & 70 & 60 & 50 & 40 & 30 & 20 & 10 & 0 & -10\end{array}$ 
$\mathrm{CDCl}_{3}, 400 \mathrm{MHz}$

4d<smiles>C#CCCCCCCCCC(C)NS(=O)(=O)c1ccccc1Cl</smiles><smiles>c1ccccc1</smiles> 
$\mathrm{CDCl}_{3}, 400 \mathrm{MHz}$
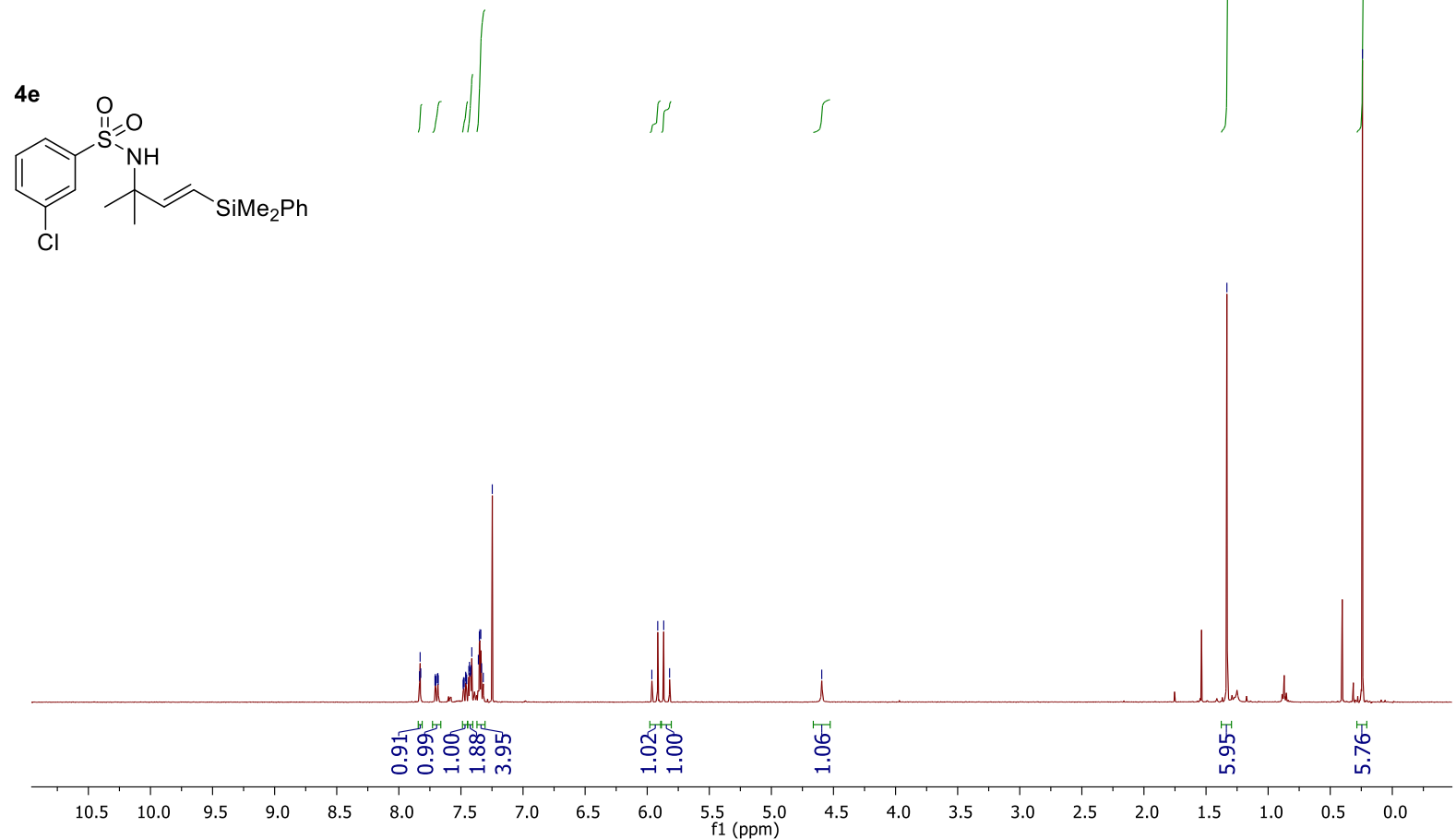

$\mathrm{CDCl}_{3}, 101 \mathrm{MHz}$

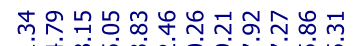

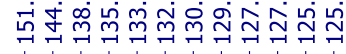

$\infty$
$\infty$
$\infty$
$\infty$
1
1

$\stackrel{\substack{0 \\ \infty}}{\stackrel{\infty}{1}}$

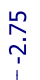

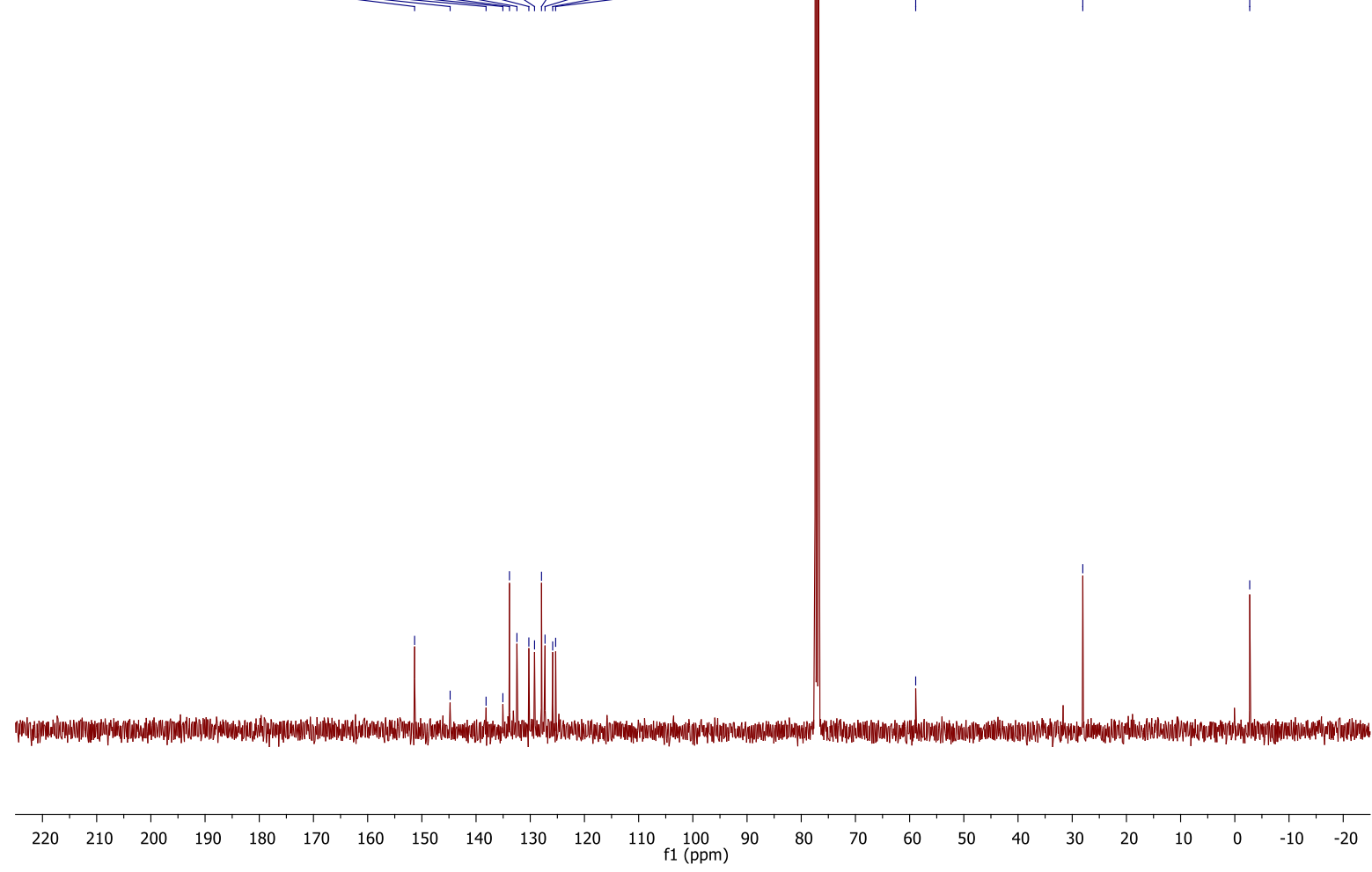




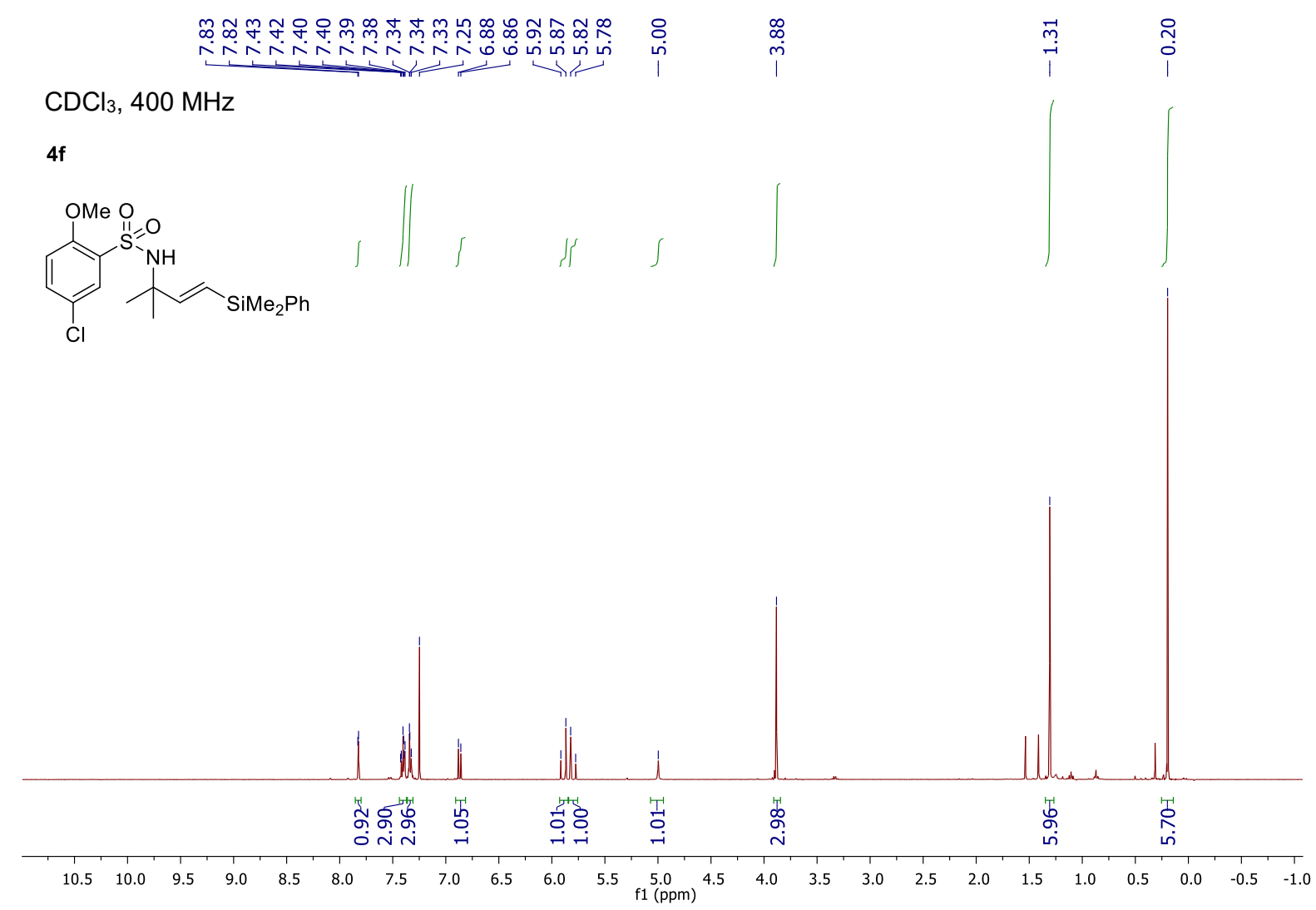

$\mathrm{CDCl}_{3}, 101 \mathrm{MHz}$

$\begin{array}{lllllllllllllllllllllllllllllllllll}220 & 210 & 200 & 190 & 180 & 170 & 160 & 150 & 140 & 130 & 120 & 110 & 100 & 90 & 80 & 70 & 60 & 50 & 40 & 30 & 20 & 10 & 0 & -10 & -20\end{array}$ 


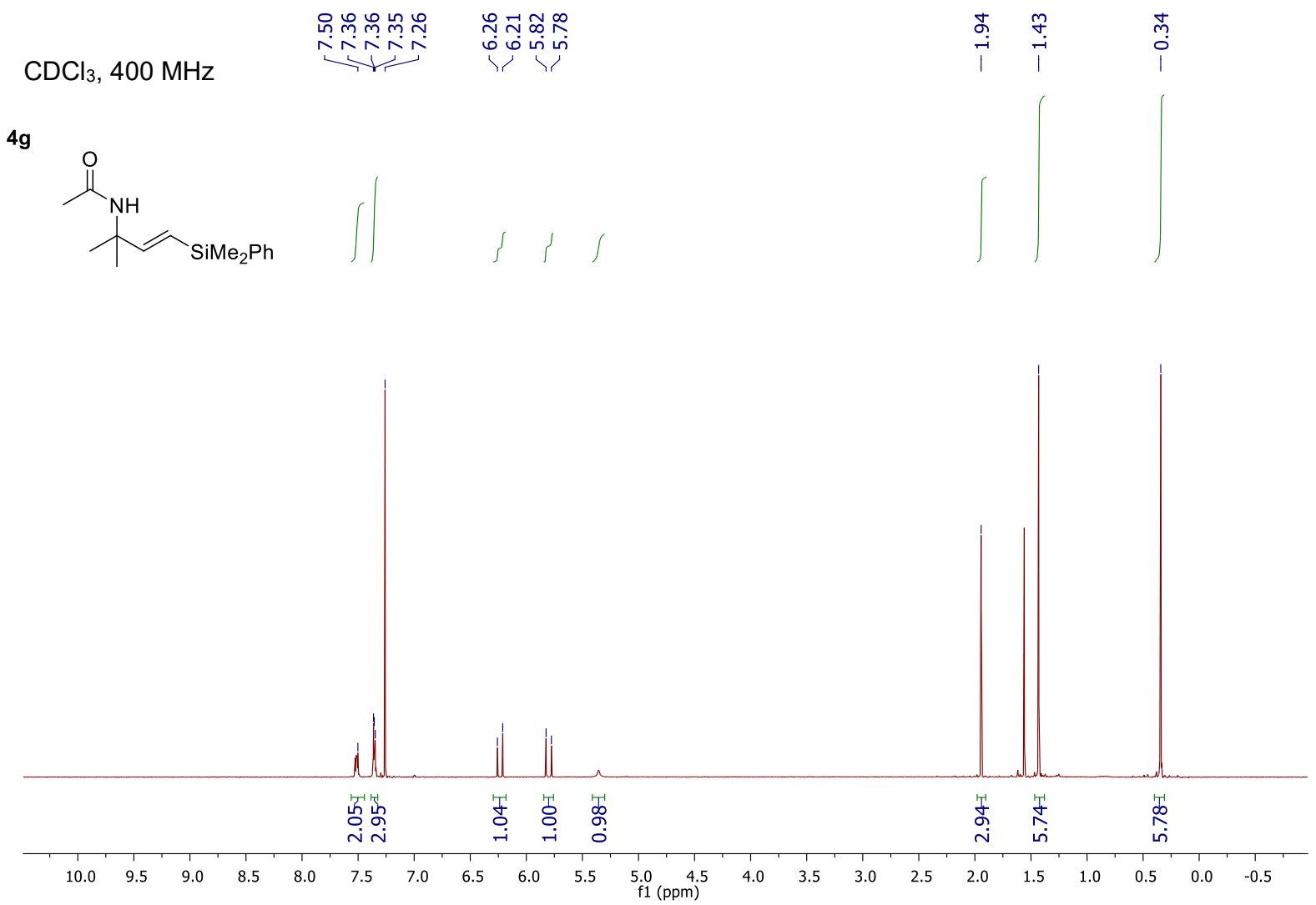

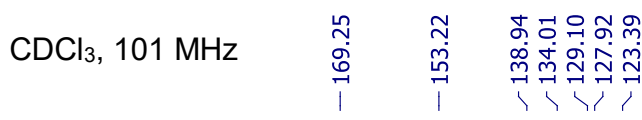

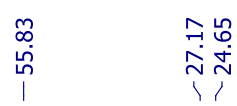

$\stackrel{\infty}{\stackrel{i}{i}}$

$\begin{array}{lllllllllllllllllllllllllllll}220 & 210 & 200 & 190 & 180 & 170 & 160 & 150 & 140 & 130 & 120 & 110 & \underset{\mathrm{f} 1}{100}(\mathrm{ppm}) & 90 & 80 & 70 & 60 & 50 & 40 & 30 & 20 & 10 & 0 & -10 & -20\end{array}$ 
$\mathrm{CDCl}_{3}, 400 \mathrm{MHz}$

$4 h$<smiles>CC(C)(C=COc1ccccc1)NC(=O)c1ccccc1Cl</smiles>
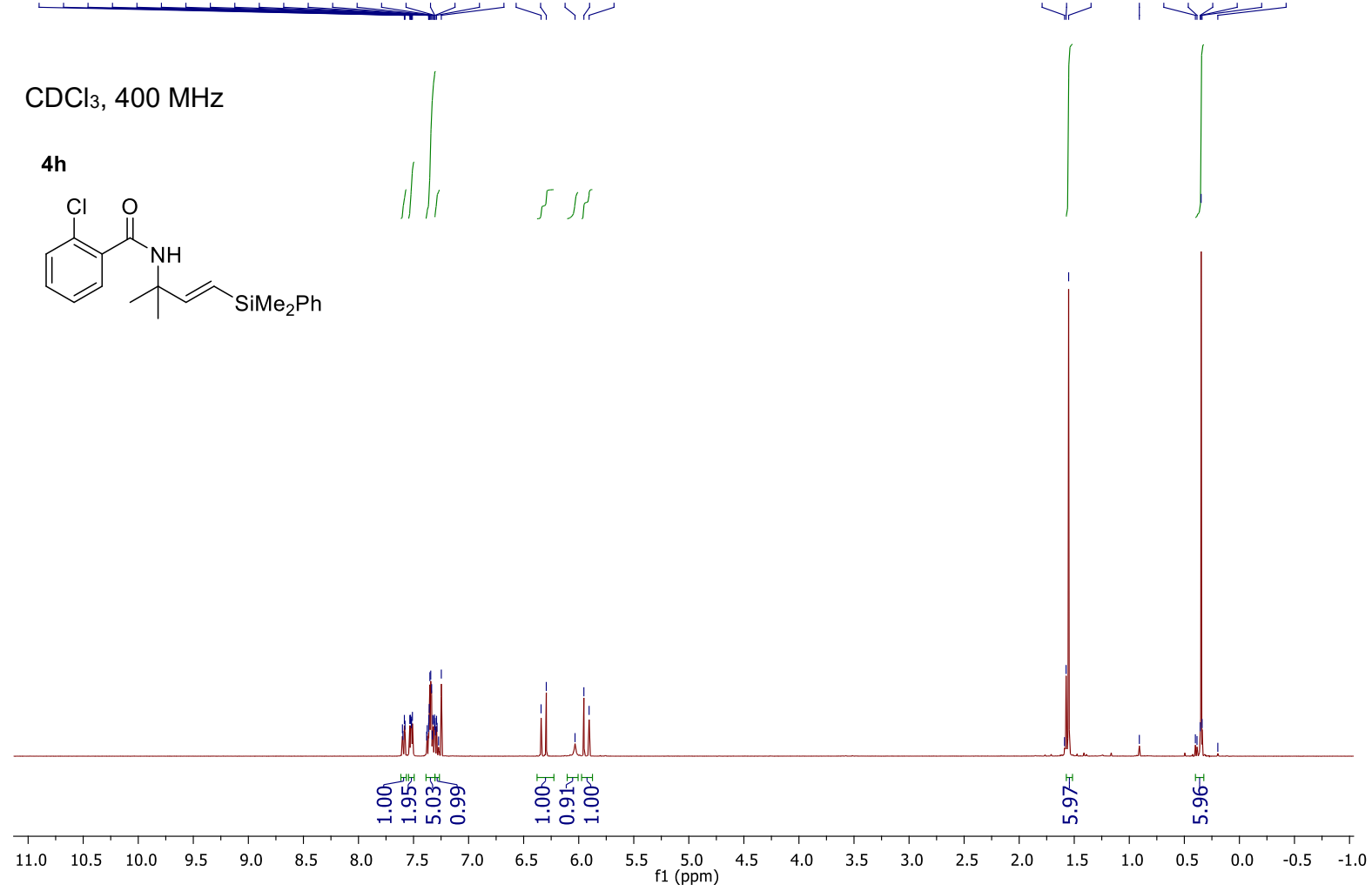

$\mathrm{CDCl}_{3}, 101 \mathrm{MHz}$

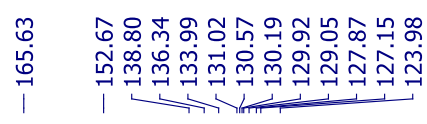

ڤิ

$\stackrel{\substack{i \\ i}}{i}$

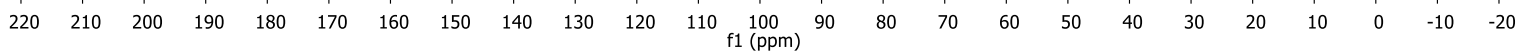


$\mathrm{CDCl}_{3}, 400 \mathrm{MHz}$

$4 \mathbf{i}$<smiles>CC(C)(C=Cc1ccccc1)NC(=O)c1ccco1</smiles>

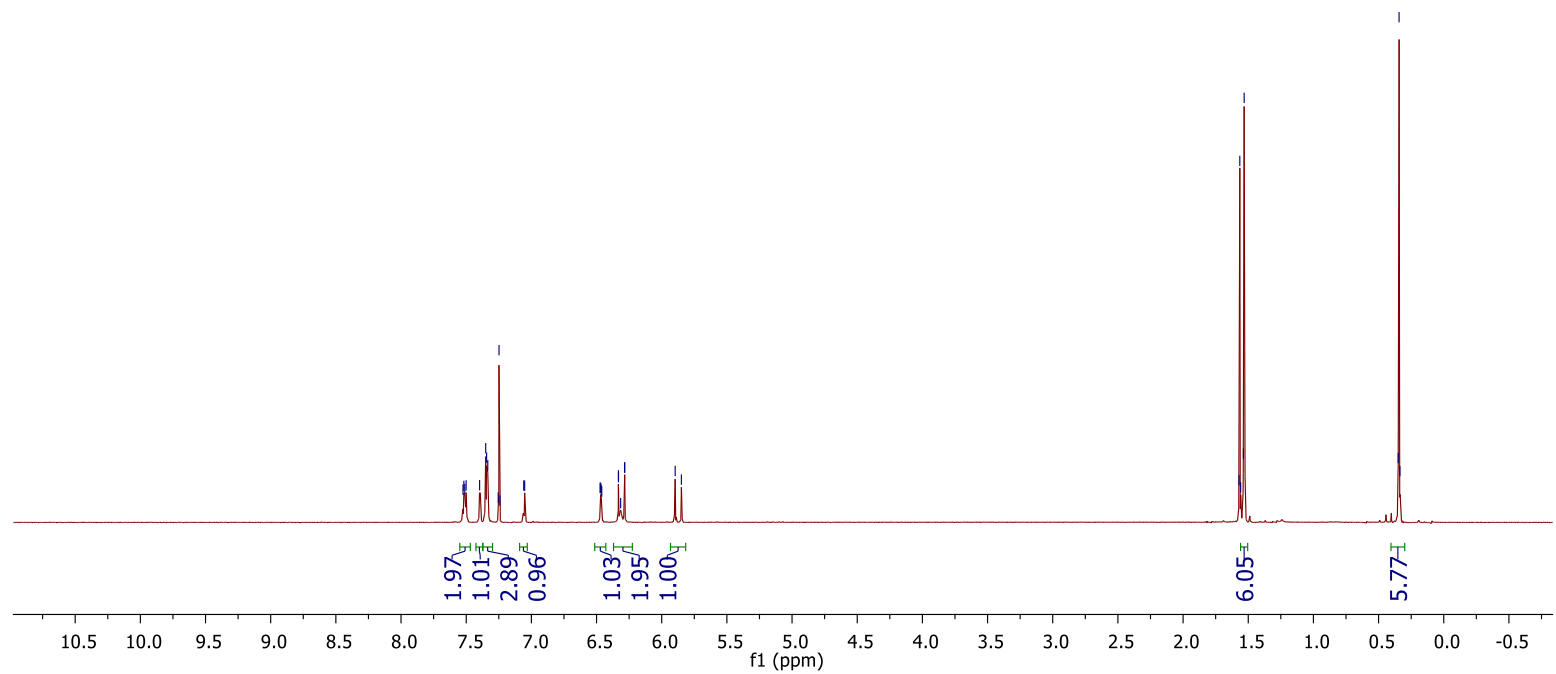

$\mathrm{CDCl}_{3}, 101 \mathrm{MHz}$

นกำ 눙 壬

ก⿱艹

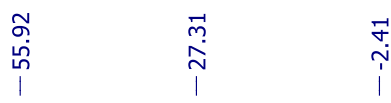

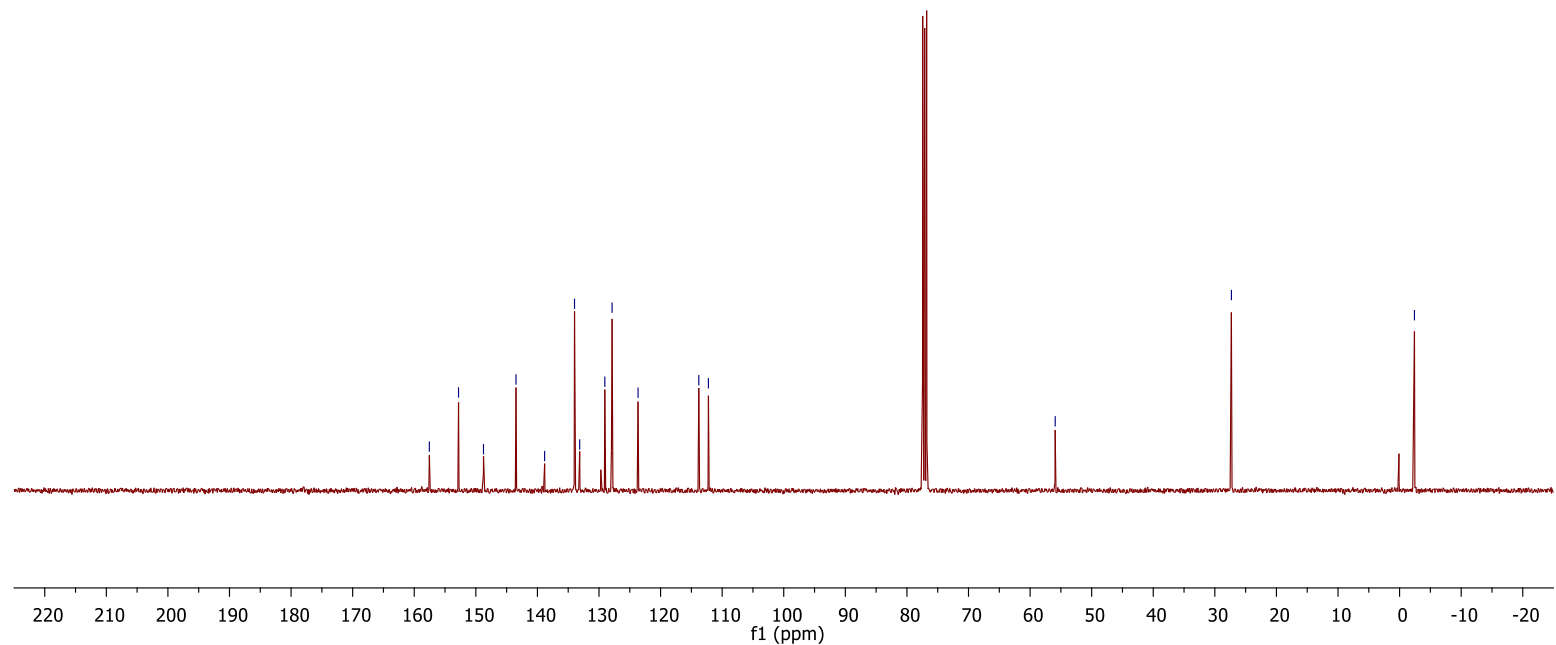




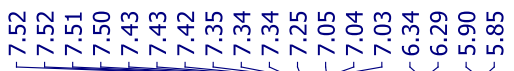

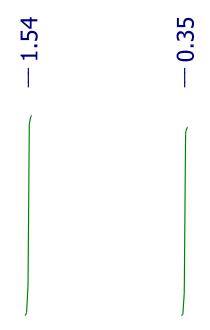<smiles>CC(C)(/C=C/Cc1ccccc1)NC(=O)c1cccs1</smiles>
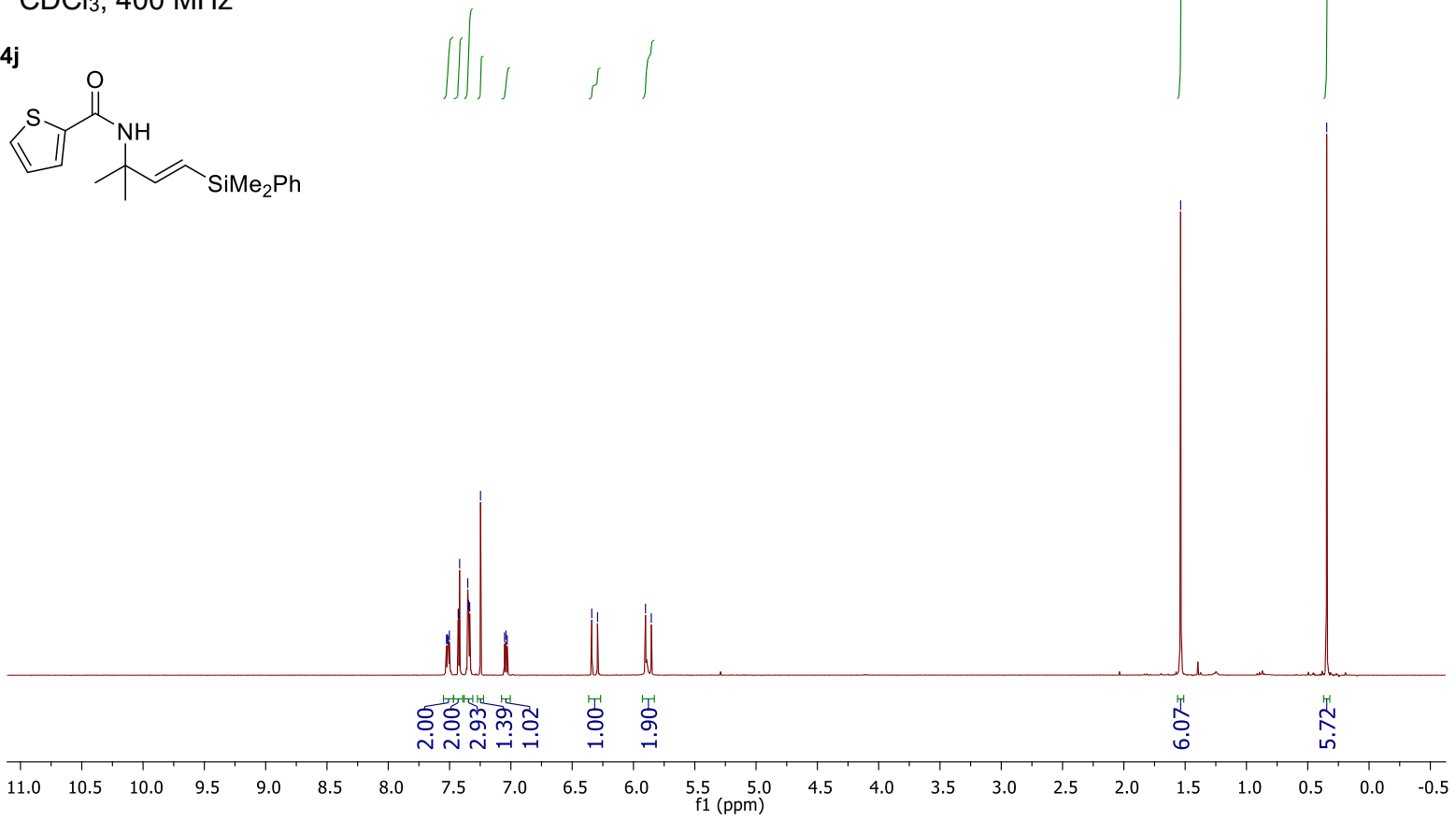

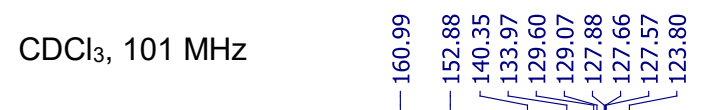
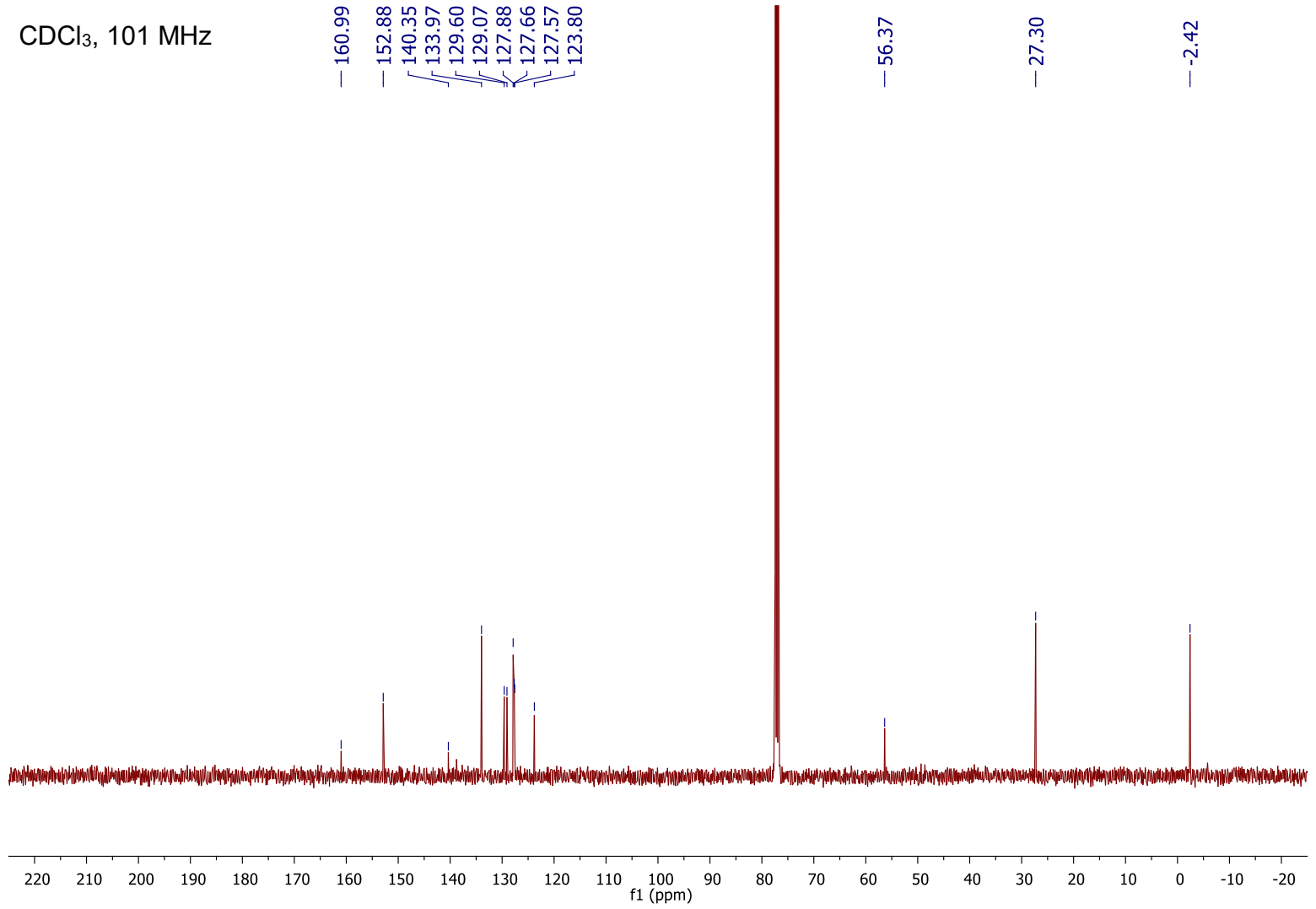

S66 


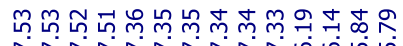

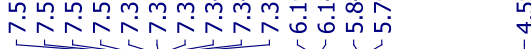

$\mathrm{CDCl}_{3}, 400 \mathrm{MHz}$

$4 k$<smiles>CC(C)(/C=C/CSc1ccccc1)NC(=O)OC(C)(C)C</smiles>

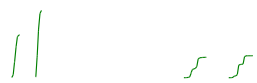

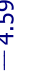

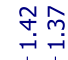

$\stackrel{+}{m}$

$\mathrm{SiMe}_{2} \mathrm{Ph}$

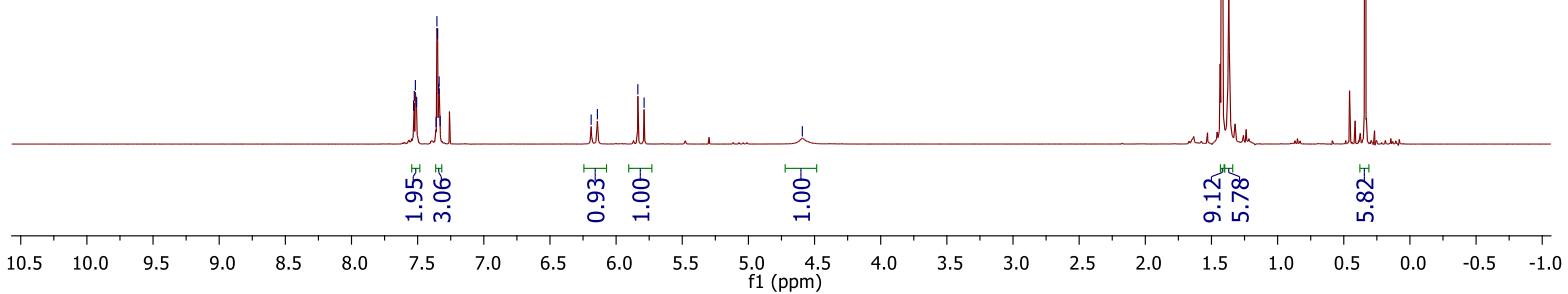

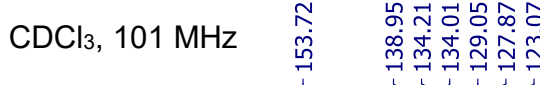

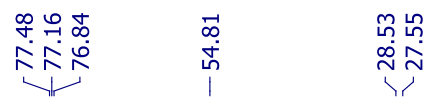

$\stackrel{m}{i}$

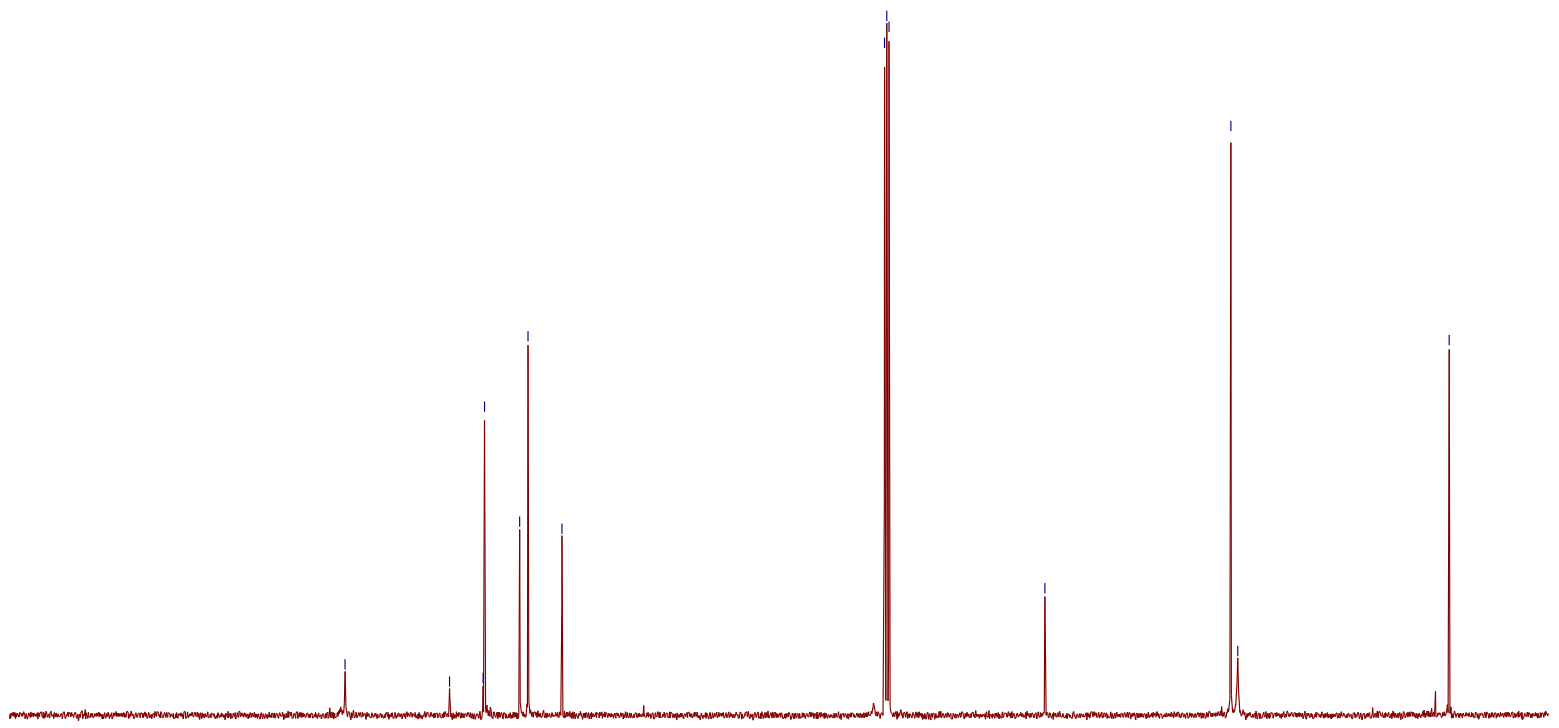



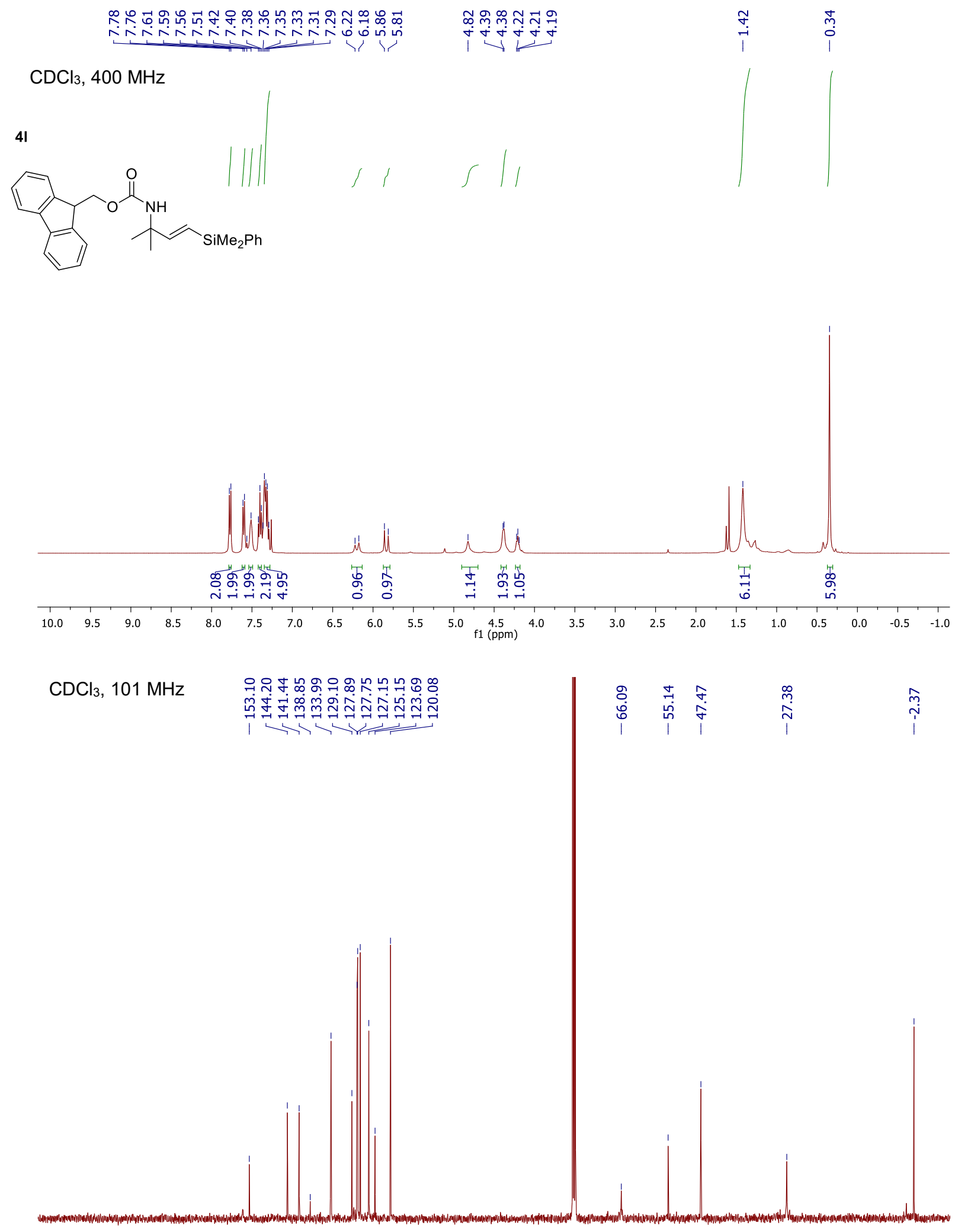

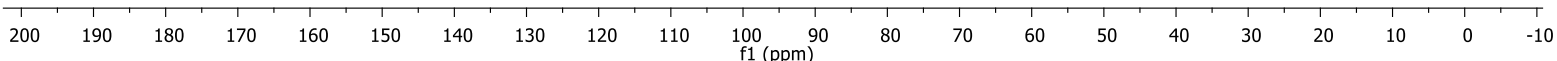




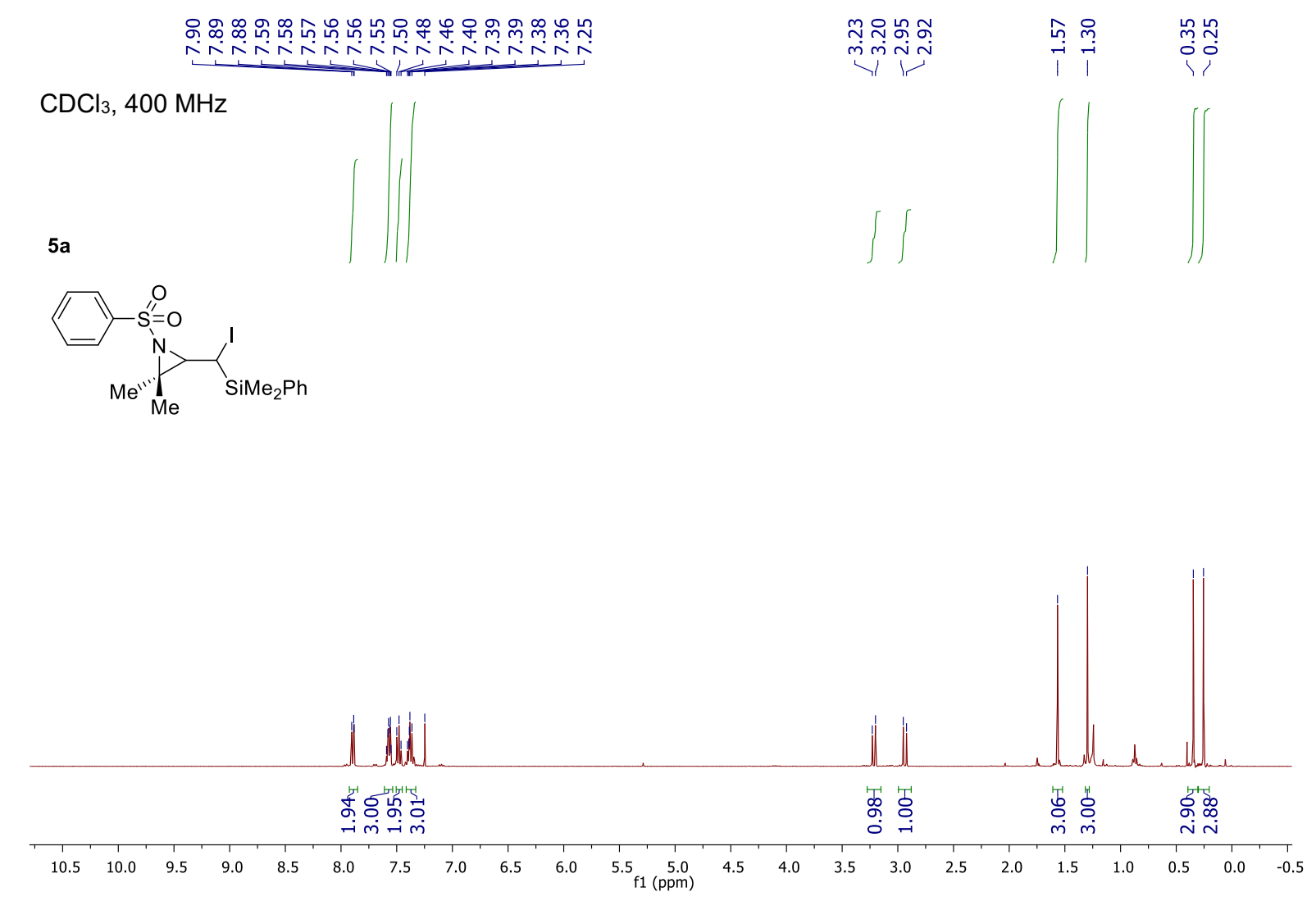

$\mathrm{CDCl}_{3}, 101 \mathrm{MHz}$

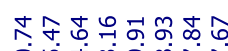

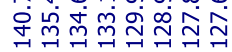

年年余 每

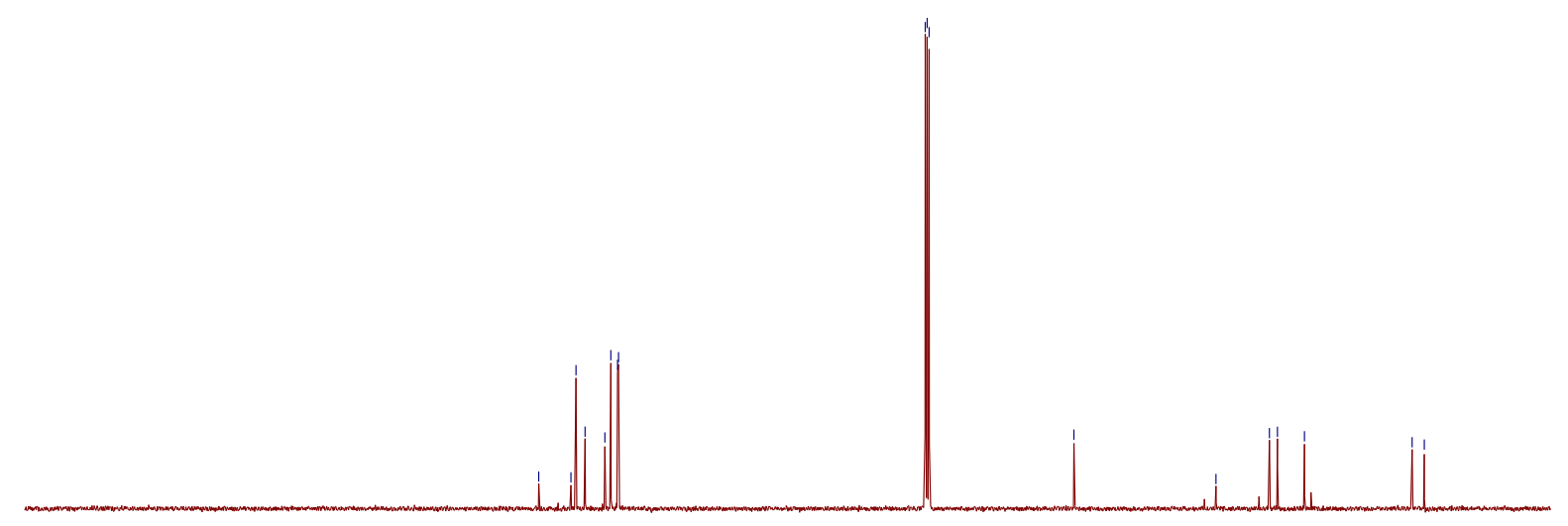

\begin{tabular}{lllllllllllllllllllllllllllllllll}
\hline & 220 & 210 & 200 & 190 & 180 & 170 & 160 & 150 & 140 & 130 & 120 & 110 & 100 & 90 & 80 & 70 & 60 & 50 & 40 & 30 & 20 & 10 & 0 & -10 & -20
\end{tabular} 

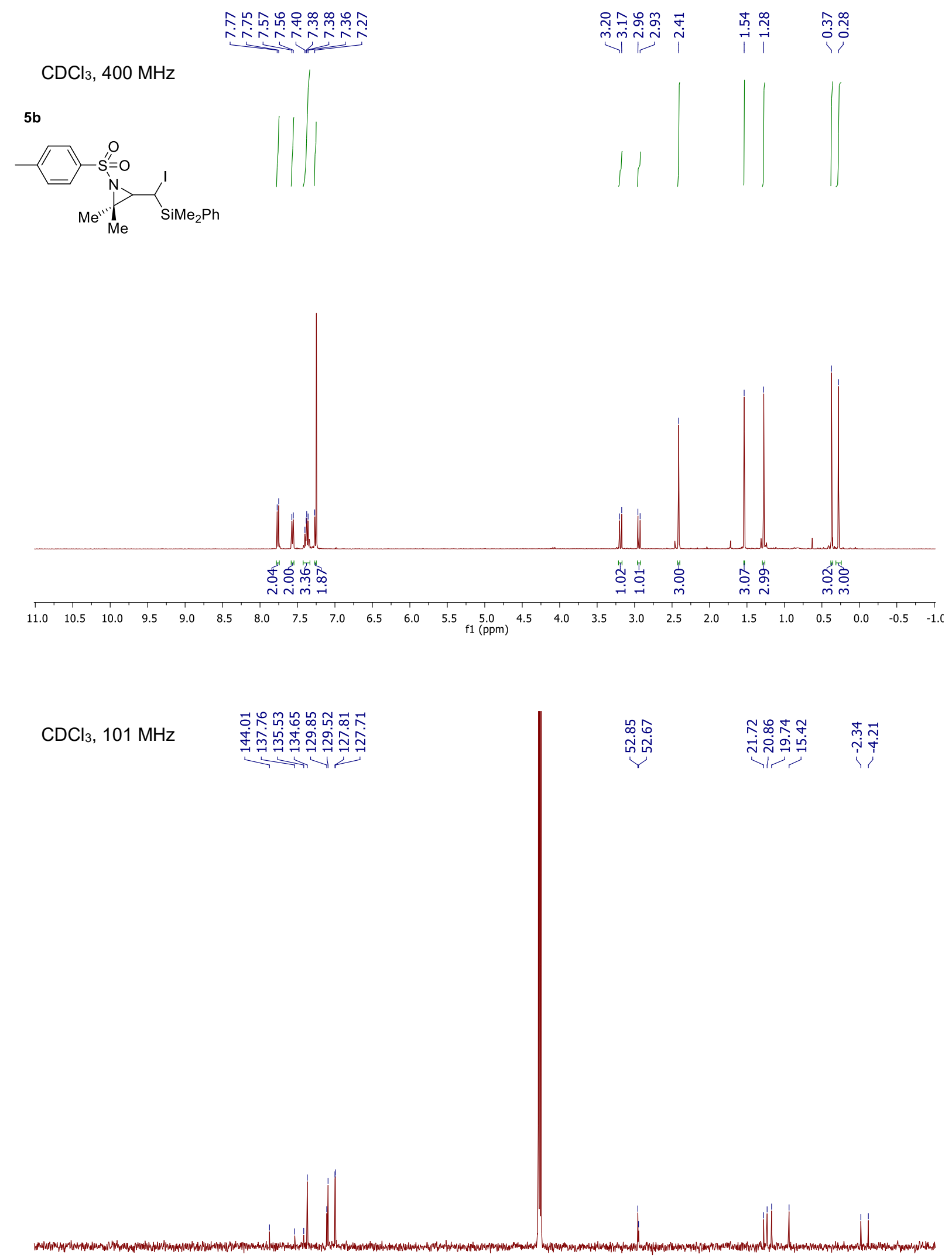

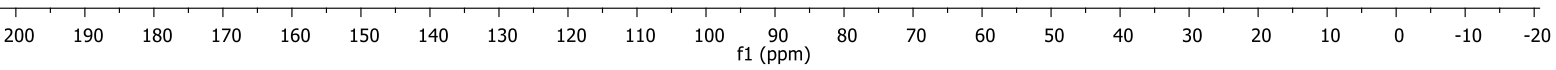




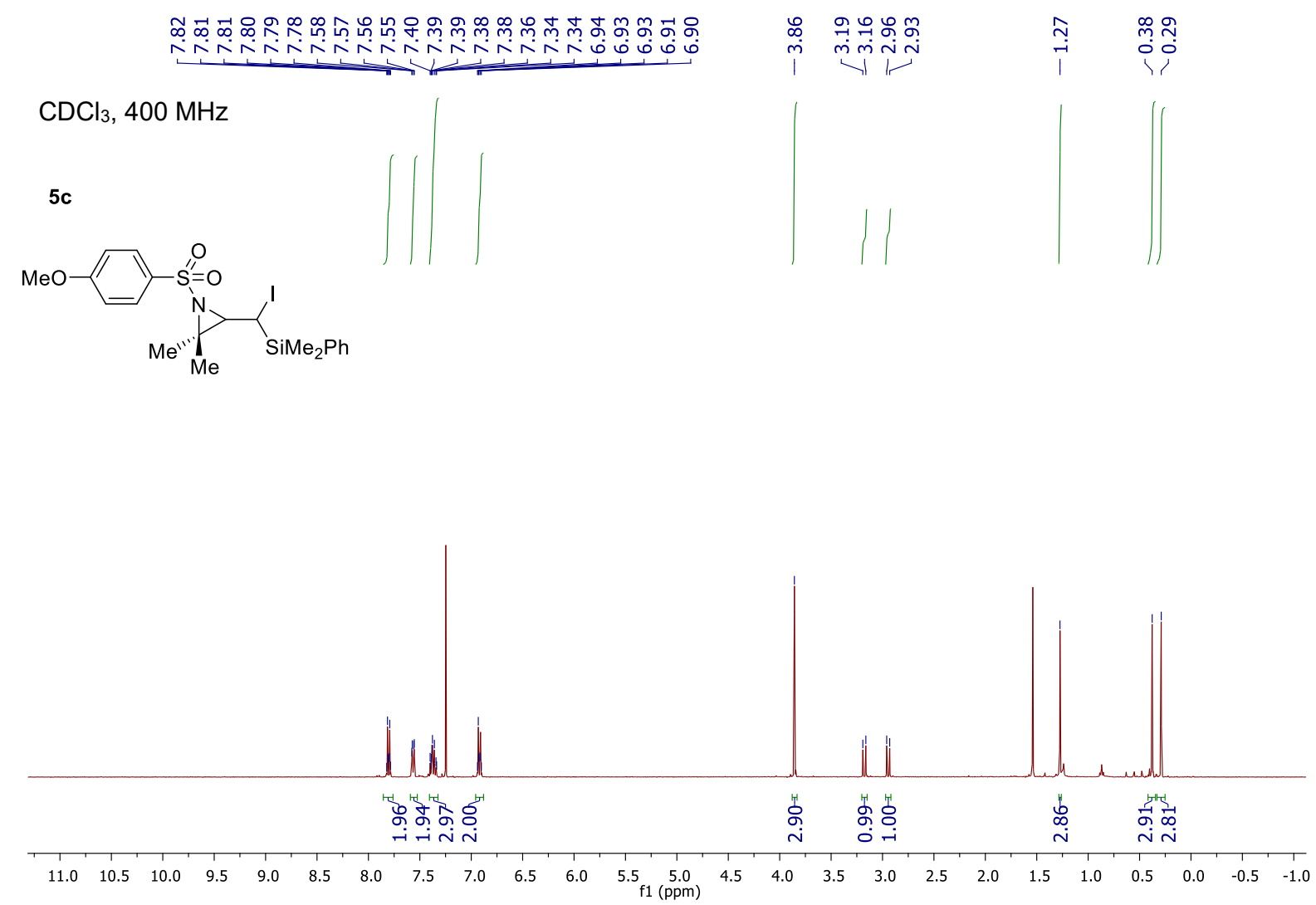

$\mathrm{CDCl}_{3}, 101 \mathrm{MHz}$

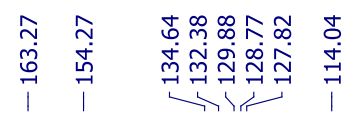

ำ

นก่ กิ่

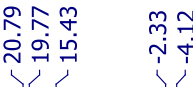

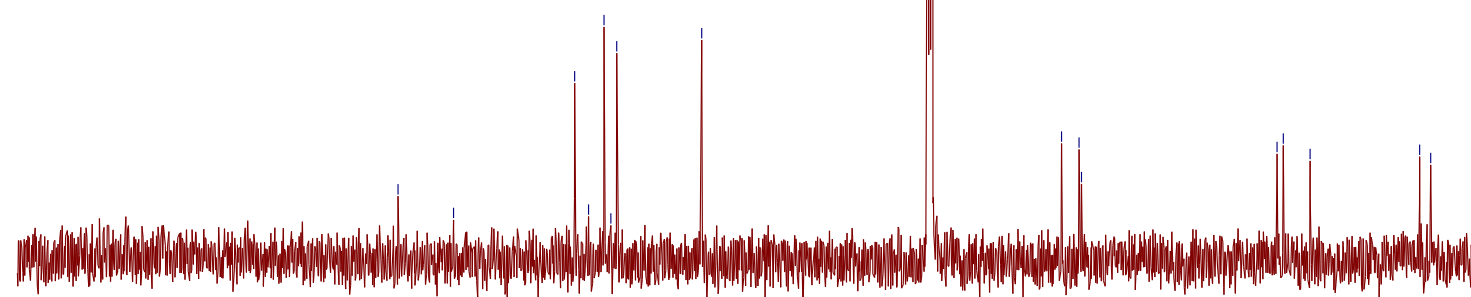

$\begin{array}{lllllllllllllllllllllllllllllllll}220 & 210 & 200 & 190 & 180 & 170 & 160 & 150 & 140 & 130 & 120 & 110 & 100 & 100 & 80 & 70 & 60 & 50 & 40 & 30 & 20 & 10 & 0 & -10 & -20\end{array}$ 

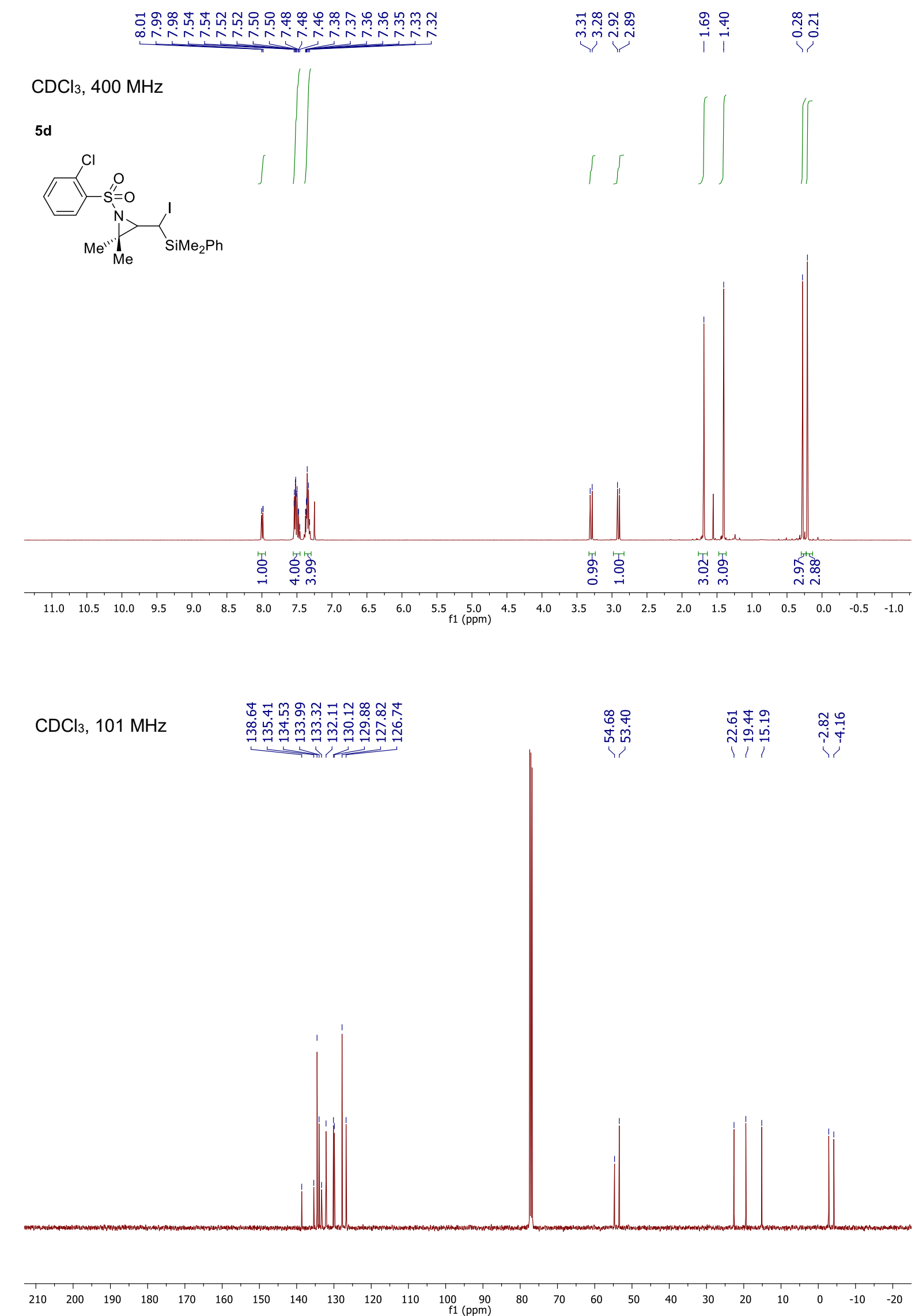


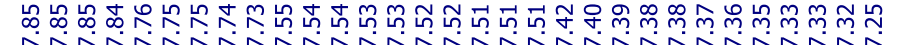

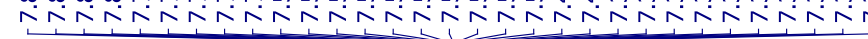

$\mathrm{CDCl}_{3}, 400 \mathrm{MHz}$

$5 e$<smiles>CC1(C)C(C(I)Sc2ccccc2)N1S(=O)(=O)c1cccc(Cl)c1</smiles>

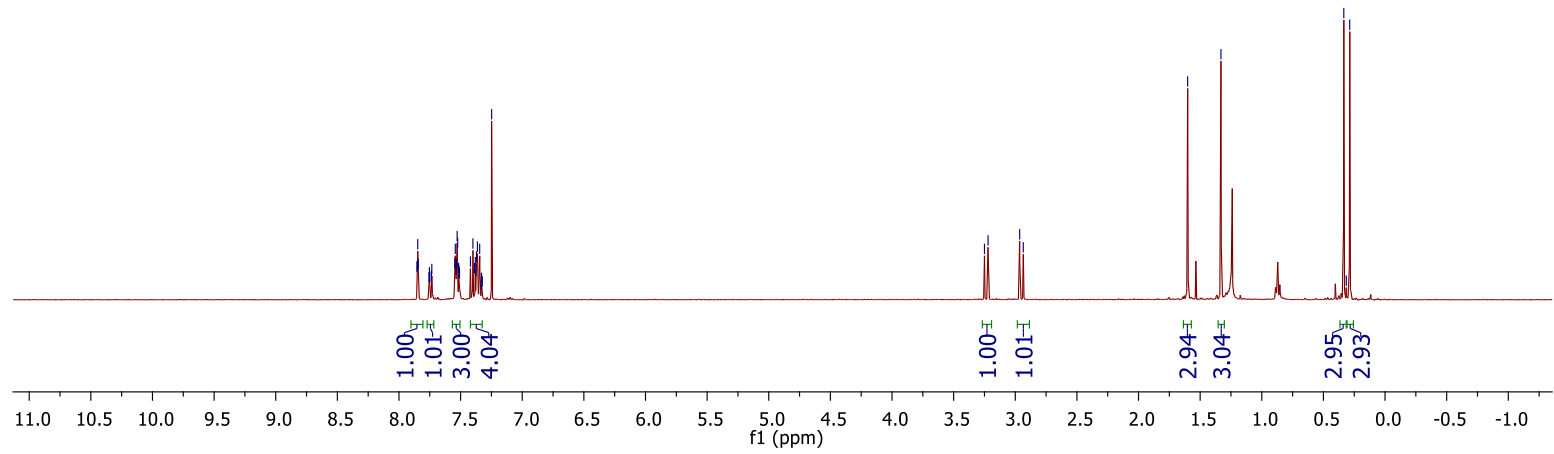

$\mathrm{CDCl}_{3}, 101 \mathrm{MHz}$

ช.

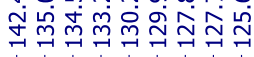

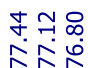

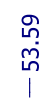

$\infty$ 구요

กิ่

ஸู่

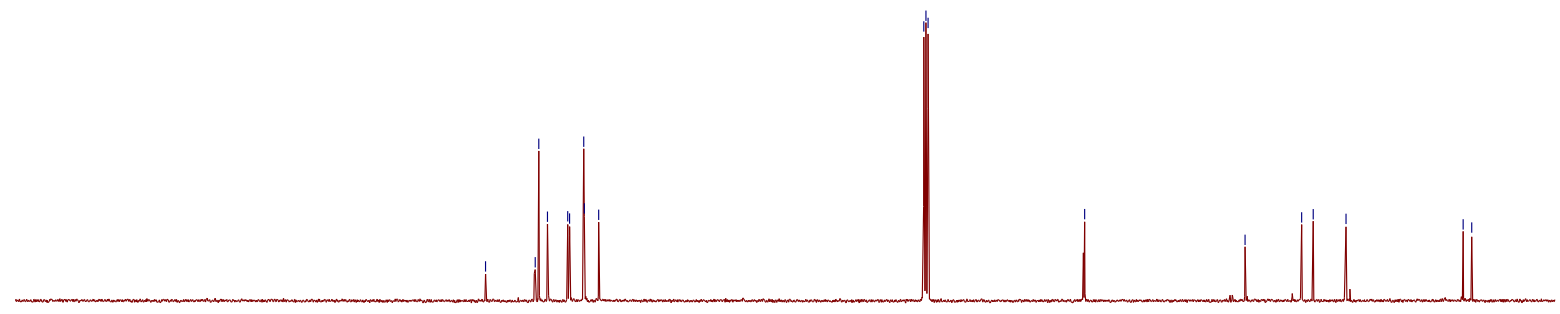

210

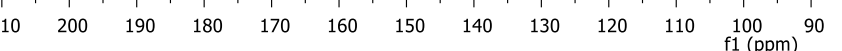


$5 f$<smiles>COc1ccc(Cl)cc1S(=O)(=O)N1C(C(I)[AsH2]c2ccccc2)C1(C)C</smiles><smiles>CC#CCCCC</smiles>

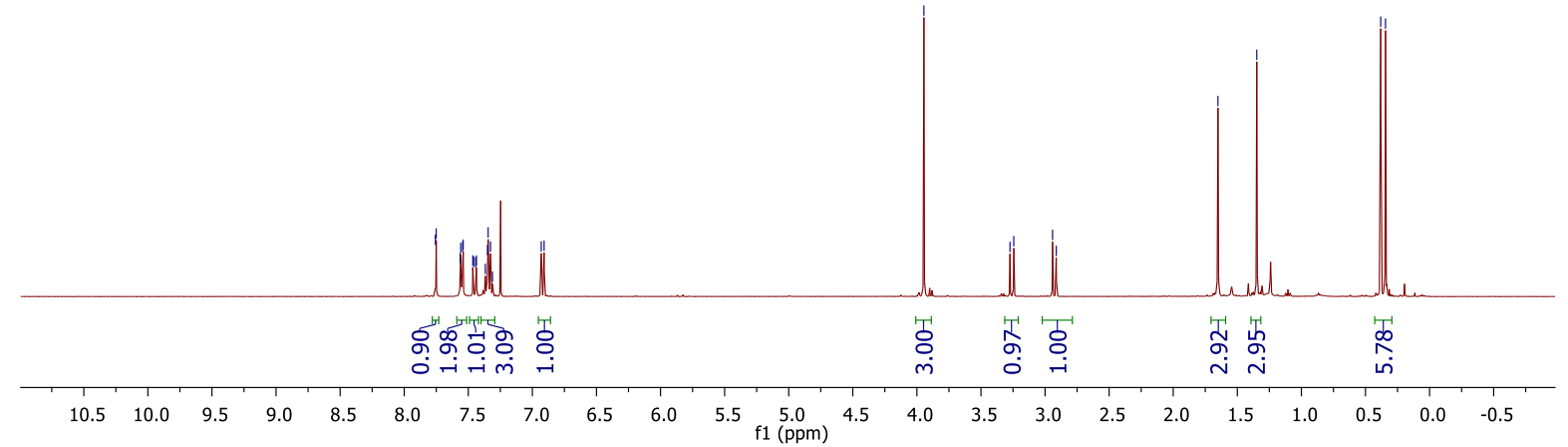

$\mathrm{CDCl}_{3}, 101 \mathrm{MHz}$

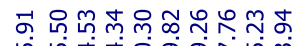

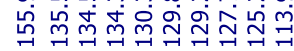




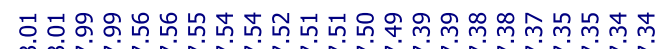

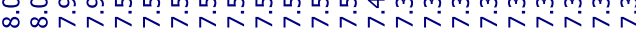

$\mathrm{CDCl}_{3}, 400 \mathrm{MHz}$

$5 g$

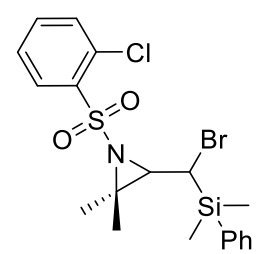

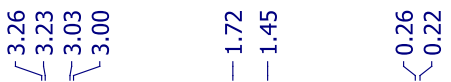

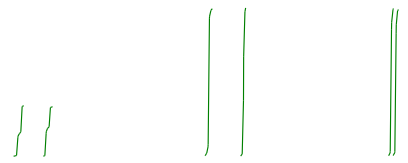

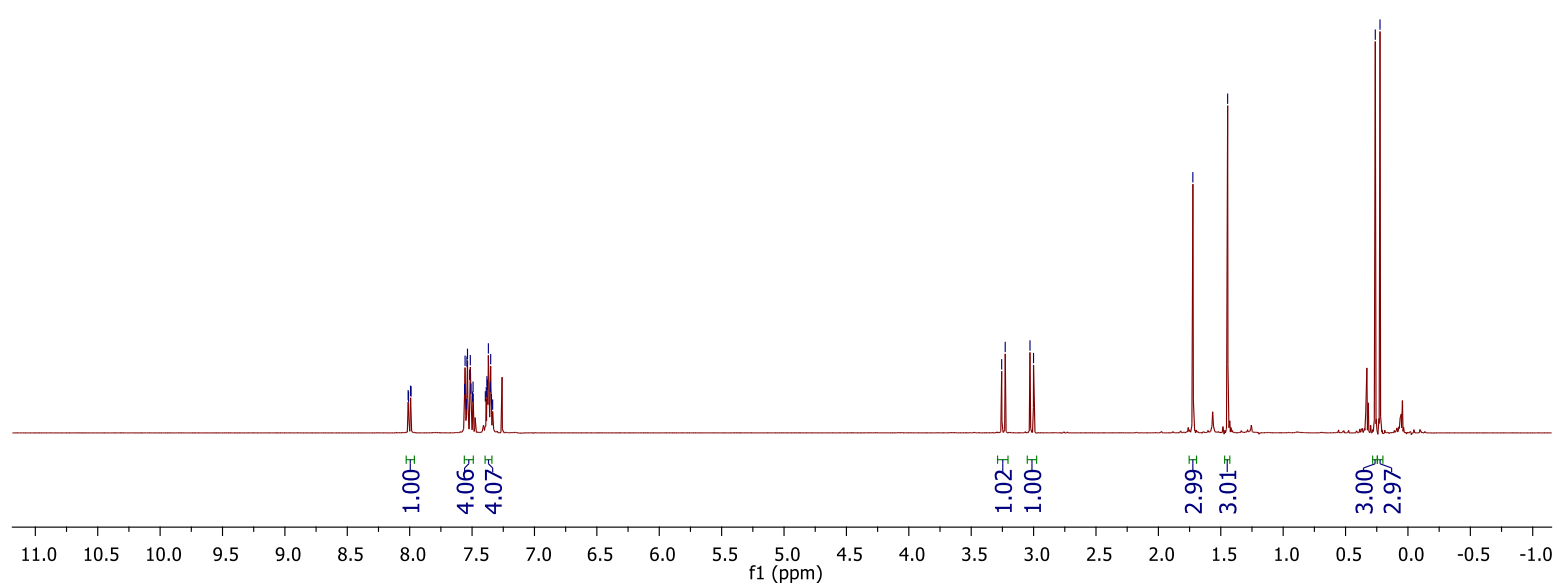

$\mathrm{CDCl}_{3}, 101 \mathrm{MHz}$

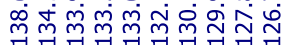

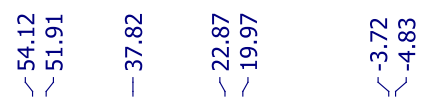

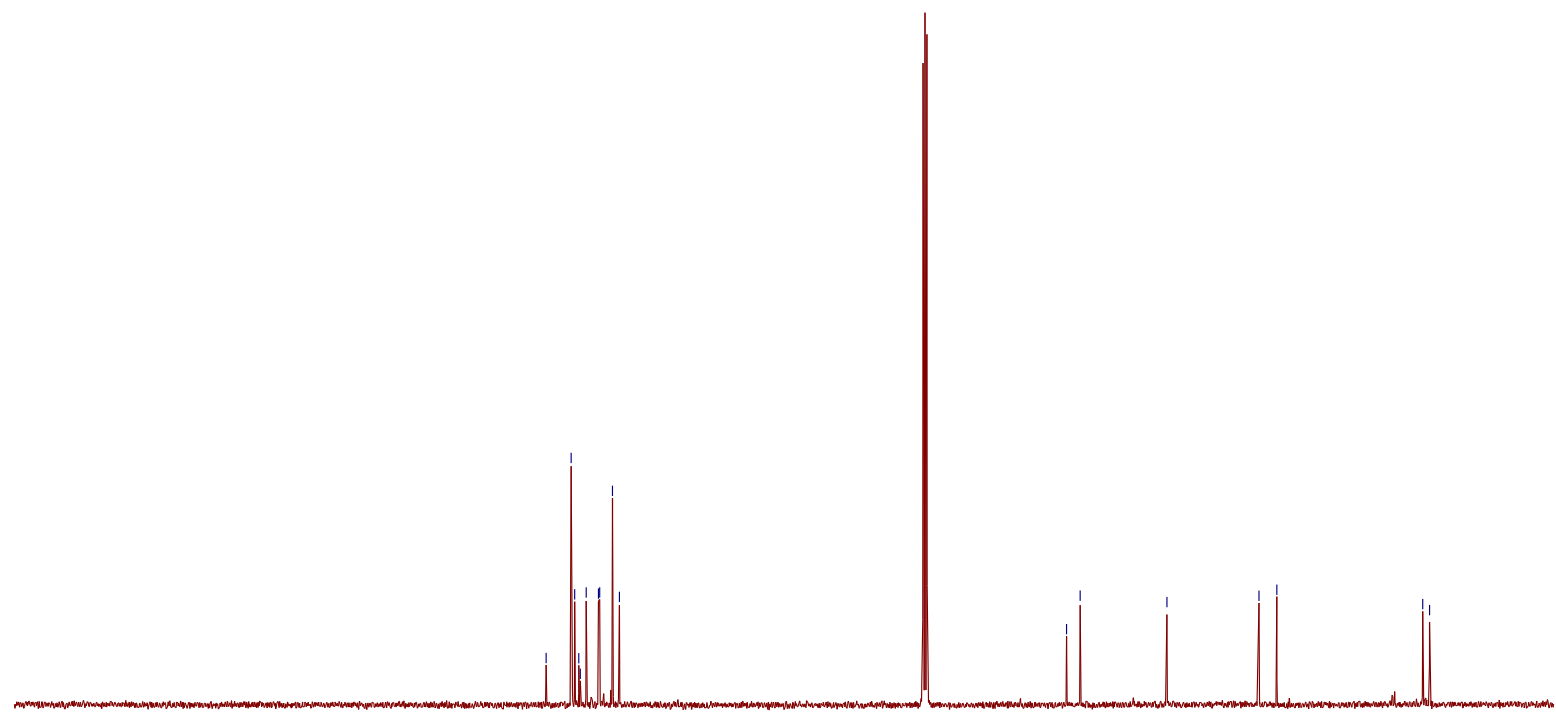

$\begin{array}{lllllllllllllllllllllllllllllllllll}220 & 210 & 200 & 190 & 180 & 170 & 160 & 150 & 140 & 130 & 120 & 110 & 100 & 90 & 80 & 70 & 60 & 50 & 40 & 30 & 20 & 10 & 0 & -10 & -20\end{array}$ 